\title{
Challenges encountered applying equilibrium and non-equilibrium binding free energy calculations
}

\author{
Hannah M. Baumann, ${ }^{\dagger}$ Vytautas Gapsys, ${ }^{\ddagger}$ Bert L. de Groot, ${ }^{\ddagger}$ and David L. \\ Mobley*,†, 9 \\ $\dagger$ Department of Pharmaceutical Sciences, University of California, Irvine \\ $\ddagger$ Computational Biomolecular Dynamics Group, Department of Theoretical and \\ Computational Biophysics, Max Planck Institute for Biophysical Chemistry, D-370ry \\ Göttingen, Germany \\ 【Department of Chemistry, University of California, Irvine \\ E-mail: dmobley@mobleylab.org
}

\begin{abstract}
Binding free energy calculations have become increasingly valuable to drive decision making in drug discovery projects. However, among other issues, inadequate sampling can reduce accuracy, limiting the value of the technique. In this paper we apply absolute binding free energy calculations to ligands binding to $\mathrm{T} 4$ lysozyme L99A and HSP90 using equilibrium and non-equilibrium approaches. We highlight sampling problems encountered in these systems, such as slow side chain rearrangements and slow changes of water placement upon ligand binding. These same types of challenges are likely to show up in other protein-ligand systems as well and we propose some strategies to diagnose and test for such problems in alchemical free energy calculations. We also explore similarities and differences in how the equilibrium and the non-equilibrium approaches handle these problems. Our results show the large amount of work still to be done to make free energy calculations robust and reliable and provide insight for future research in this area.
\end{abstract}

\section{Introduction}

In drug discovery, computational methods help navigate through a vast potential chemical space. There is often a trade-off between accuracy and speed of the methods which determines the value of each technique at different stages of the discovery process. A common goal in drug discovery is to search for molecules which bind to proteins with therapeutic potential.

In order to explore a binding site of interest and find putative binders, large chemical libraries like Enamine, with 1.36 billion molecules, ${ }^{1}$ can be scanned. ${ }^{2}$ Fast and less accurate methods, such as ligand-based approaches, molecular docking and physicochemical property prediction, are used to filter this large space. Promising compounds can then be purchased, tested and active compounds improved iteratively.

In lead optimization, experiments may require difficult synthesis and thus be costly and slow. Therefore, during optimization, more accurate, but also more computationally expensive methods, such as free energy calculations, are used to prioritize the synthesis of compounds based on the in silico predictions. Although all these methods are routinely applied in drug discovery projects, improvements in their accuracy are still needed to increase their impact. ${ }^{3-6}$ 
The binding free energy, $\Delta G^{\circ}$, is defined as the difference between the free energy of the ligand in solution at a standard reference concentration, and the free energy of the ligand bound to the protein. This property can be calculated in a variety of ways, but two - which we consider here - are becoming relatively more common. In the so-called "absolute" approach, the $\Delta G^{\circ}$ is calculated for each ligand independently. Alternatively, the "relative" approach determines the difference in binding free energy, $\Delta \Delta G^{\circ}$, between the binding of two ligands. In the latter case, commonly only atoms which differ between the two molecules are transformed, and the absolute binding free energy can be obtained if the binding free energy of one of the ligands is known. This relative approach, then, is most suitable for comparing or ranking binding of related ligands.

Absolute binding free energy (ABFE) calculations have some benefits over relative ones (RBFE), ${ }^{5,7}$ but are thought to require more sampling because of the generally larger transformations involved compared to RBFE. In ABFE calculations, ligands of interest can be very chemically diverse since they need not share a common scaffold. In addition, different ligands need not share a common binding mode. The method can even be used for selectivity predictions, predicting binding of a ligand to multiple proteins. ${ }^{8}$ However, ABFE calculations are thought to require more sampling and simulations may take a long time to converge which can make them computationally expensive. ${ }^{5}$ ABFE calculations in some systems likely require additional sampling of slow protein motions relative to RBFE calculations, such as changes in protein conformation which occur on ligand binding (e.g. HIV protease flap opening/closing, ${ }^{9}$ kinase activation loop, ${ }^{10}$ etc.).

Alchemical free energy calculations employ an unphysical path that connects two physical end states in order to obtain free energy differences. ${ }^{11-17}$ Let us consider for example the process of turning the interactions of a ligand off in the binding site. The initial state in this case is the fully interacting ligand bound to the protein and the final state is the decoupled ligand, not interacting with its surroundings, and a sepa- rate protein and solvent box. Here the unphysical path describes how the electrostatic and steric interactions between the ligand and the protein and surrounding solvent molecules are gradually turned off. The unphysical intermediates ensure sufficient overlap of phase space as the system is modified, allowing for calculation of accurate free energies. The Hamiltonian of the system is coupled to a parameter $\lambda$ which controls the progress between the physical end states. $\lambda$ can have discrete or continuous values between 0 (initial state) and 1 (final state) and the intermediate states are often referred to as $\lambda$ windows. Different alchemical approaches have been developed, each having its strengths and weaknesses.

In equilibrium free energy calculations (EQ) a separate molecular dynamics (MD) simulation is performed for each of a set of discrete $\lambda$ windows corresponding to different states of the system (Figure 1). This method often requires long simulation times since every state has to first reach equilibrium before data can be used for the analysis. If this is not achieved - and it can be difficult to ensure that it has been achieved - the approach can suffer from systematic errors. ${ }^{18}$

In contrast, with a non-equilibrium approach (NEQ), only the physical end states (i.e. the interacting state and the noninteracting state) have to be sampled at equilibrium while the alchemical path is explored in fast switching transitions, as the ligand is switched from interacting to non-interacting (or vice versa) in a single simulation. The coupling parameter $\lambda$ is continuous in this case. Multiple trials are performed, and the exponential average over the non-equilibrium work necessary to transform the ligand is related to the free energy difference of the process by the Jarzynski equality ${ }^{19}$ (or, better if transformations in both directions are possible, the Crooks formalism ${ }^{18,20}$ ).

Another potential benefit of the NEQ approach is that it is highly parallelizable which can reduce wall clock time when distributed computing is available. In NEQ calculations, end state simulations can also benefit from enhanced sampling techniques ${ }^{21}$ to overcome energy barriers. Intuitively such strategies seem 
likely to be beneficial. However, recent literature $^{22}$ using partial replica exchange MD (an enhanced sampling techique involving exchanges between replicas with modified Hamiltonians) showed no effect of the enhanced sampling. Work in preparation from Gapsys and de Groot also suggests that in some cases, enhancing the sampling of the apo/holo states can drive the system away from the starting crystallographic pose by accumulating force field artifacts and equilibrating in a new free energy minimum which may differ from the experimental one.

While most literature applications of free energy calculations have used EQ or NEQ approaches, a third possibility is expanded ensemble simulations and related approaches, such as $\lambda$ dynamics. ${ }^{23}$ Here, the parameter $\lambda$ is a dynamic variable which is propagated throughout a single simulation. These calculations are not our focus here, but provide an interesting alternative.

In this paper, we test the non-equilibrium approach for absolute binding free energy calculations and compare it with equilibrium free energy calculations. We highlight challenges encountered applying the two methods and explore similarities and differences in handling these issues. Since the challenges we identify are likely to show up in other protein-ligand systems as well, we propose some strategies to assess sampling problems in alchemical free energy calculations (FEC). Although we find ways to work around some of the problems, our results highlight the large amount of work still to be done to make these calculations robust and to reliably detect problems in an automated way. Most literature studies report only successes, and calculations generally seem to work, though industry reports have highlighted the need for more careful analysis of failures. ${ }^{4}$ Here, we report on a relatively large number of "failures" with common protocols and highlight how they fail and why. We provide insights and directions for future method development and research in this area.

\section{Theory}

2.1. Alchemical free energy calculations are a physically rigorous approach to calculate binding free energies. The strength of protein-ligand interactions can be measured by the binding affinity or the related quantity, the binding free energy, given by:

$$
\Delta G^{\circ}=-k_{B} T \ln \left(c^{\circ} K_{B}\right)=-k_{B} T \ln \left(c^{\circ} \frac{[P L]}{[P][L]}\right)
$$

where $k_{B}$ is the Boltzmann constant, $T$ the temperature, $K_{B}$ the binding affinity, $c^{\circ}$ the standard state concentration of $1 \mathrm{~mol} / 1$ and $[P L]$, $[P],[L]$ the concentrations of the protein-ligand complex, the protein and the ligand, respectively.

Binding free energy calculations are based on the relationship between the free energy and the configurational integral (partition function). ${ }^{24,25}$ This partition function cannot be calculated explicitly for complex systems, but can be approximated by an ensemble of conformations from the Boltzmann distribution. In practice, molecular dynamics or Monte Carlo simulations are used to sample the configurational space. Several approaches serve to analyze the resulting data and obtain the change in free energy. $^{26}$

2.2. Equilibrium free energy simulations require multiple separate intermediate states. For the EQ approach, the Bennett Acceptance Ratio (BAR) ${ }^{27}$ and its variant the Multistate BAR (MBAR) ${ }^{28}$ are the most efficient estimators.

As input, BAR and MBAR take potential energy differences between adjacent alchemical states. These potential energy differences can be written (in "reduced" / dimensionless form) as $\Delta u_{i j}$ where $i$ and $j$ denote the thermodynamic states in question. Particularly, these states correspond to different intermediate states or $\lambda$ windows per the Introduction.

The potential energy differences $\Delta u_{i j}$ are evaluated for samples taken from a single simulation and assess the work which would be required to move the system from one state (or one Hamiltonian) to the other. A simulation 
in state $i$ can be said to have coordinates $x_{i}$, and from that simulation we evaluate $\Delta u_{i j}\left(x_{i}\right)$, the energy difference to alter the energy function to that corresponding to state $j$. Likewise, we also use snapshots from the simulation run in state $j$ to compute $\Delta u_{i j}\left(x_{j}\right)$. Here, $\Delta u_{i j}(x)=u_{j}(x)-u_{i}(x){ }^{26}$

In this notation, the potential energy $u_{i}(x)$ refers to a dimensionless reduced potential function that includes the pressure-volume work; this allows easier accommodation of the equations to different choices of ensemble; here, calculations are performed in the isothermalisobaric ensemble: ${ }^{28}$

$$
u_{i}(x)=\beta\left(U_{i}(x)+p_{i} V(x)\right)
$$

where $x$ is the configuration of the system, $V(x)$ the volume and $U_{i}(x)$ the potential energy function.

Overall in BAR, the free energy difference is obtained by numerically solving the following equation for $\Delta G$ :

$$
\sum_{i=1}^{n_{i}} \frac{1}{1+e^{\left(M+\Delta u_{i j}\left(x_{i}\right)-\Delta G\right)}}=\sum_{j=1}^{n_{j}} \frac{1}{1+e^{-\left(M+\Delta u_{i j}\left(x_{j}\right)-\Delta G\right)}}
$$

with $M=\ln \frac{n_{i}}{n_{j}}$ and $n_{i}$ and $n_{j}$ being the number of samples at state $i$ and $j$ and $\beta=1 / k_{B} T$. MBAR generalizes this expression to multiple thermodynamic states rather than pairs of states as in BAR.

The sum of all free energy differences $\Delta G$ between the $\lambda$ windows gives then the total free energy difference of the process.

In the case of BAR, only potential energy differences of adjacent states are computed while in the case of MBAR all states are considered and analyzed. MBAR provides a more generalized version of BAR which uses all available data to compute free energy estimates, rather than relying on information only from adjacent thermodynamic states.

It is important to mention that these estimators rely on phase space overlap between adjacent $\lambda$ windows to obtain reliable free energy estimates (Figure 1).

Thermodynamic integration (TI) provides another common approach for EQ calculations. ${ }^{29}$
Here, the free energy difference between two states is defined as

$$
\Delta G=\int_{0}^{1}\left\langle\frac{\delta U(\lambda)}{\delta \lambda}\right\rangle_{\lambda} d \lambda
$$

The alchemical path is sampled at a set of discrete $\lambda$ values. Therefore, this integral has to be solved numerically which introduces a bias especially when there is (strong) curvature along the alchemical pathway. ${ }^{30}$

Since sufficient sampling in all $\lambda$ states is necessary to obtain reliable free energy estimates for both estimators, enhanced sampling techniques, such as Hamiltonian Replica Exchange (HREX), ${ }^{31-33}$ are often used to improve the sampling of configuration space. Here, $\lambda$ windows are run in parallel, so that the simulations can swap configurations of the system between the states. Energy barriers of the potential energy surface might be crossed more often and earlier since the energy landscape differs among the states of the alchemical pathway. The Metropolis acceptance criterion ensures that simulations in all states still converge owards the equilibrium distribution. ${ }^{32}$

As mentioned in the introduction, all $\lambda$ windows need to be simulated at equilibrium in EQ calculations, and some of these can require sampling unphysical states which might have even slower correlation times than the physical states, ${ }^{34-36}$ necessitating very long sampling times despite the use of enhanced sampling techniques.

\subsection{Non-equilibrium calculations drive} rapid transitions between physical end states. In contrast, non-equilibrium FEC sample only the physical end states at equilibrium while the alchemical path is explored in fast (non-equilibrium) transitions. After generating an ensemble of equilibrium conformations in the initial and final state, MD simulations are performed which rapidly switch the ligand from interacting to non-interacting and vise versa (Figure 1). By changing the parameter $\lambda$, the system is forced to quickly change to the other end state without reaching equilibrium at any intermediate state. Consequently, there is dissipated work along the path and the average 

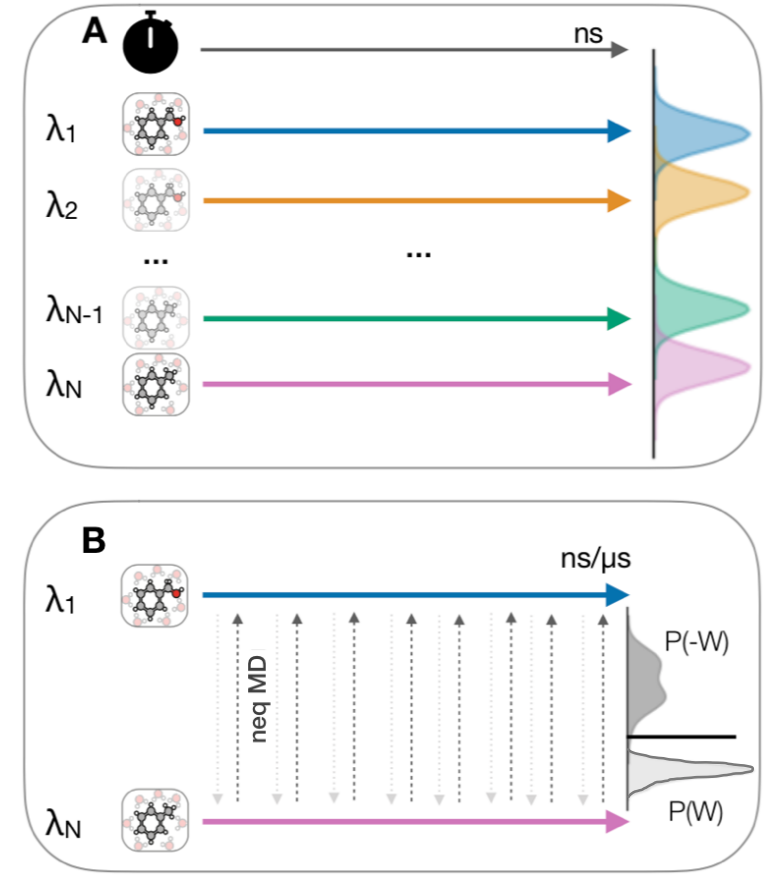

Figure 1. Protocols for the equilibrium (EQ) and nonequilibrium (NEQ) free energy calculations. (a) EQ sampling scheme. A separate molecular dynamics (MD) simulation (colored arrows) is performed for each of a set of discrete $\lambda$ windows corresponding to different states of the system $\left(\lambda_{1}-\lambda_{N}\right)$. (b) NEQ approach, where two equilibrium simulations at the physical end states are run $\left(\lambda_{1}\right.$ and $\lambda_{N}$, blue and pink arrow). The alchemical path is explored in fast switching transitions in a single simulation (grey arrows). In the bidirectional approach, many trials are performed in both directions and the non-equilibrium work distributions of the forward and reverse direction $(\mathrm{P}(\mathrm{W})$ and $\mathrm{P}(-\mathrm{W}))$ are directly related to the free energy difference between the states (thick horizontal line between the distributions) by the Crooks fluctuation theorem. Figure was adapted from Mey et al. ${ }^{14}$ under the CC-BY 4.0 license.

work exceeds the free energy difference according to the second law of thermodynamics and the maximum work theorem

$$
\langle W\rangle \geq \Delta G,
$$

where $\mathrm{W}$ is the reduced work that includes the pressure-volume work similarly as described for the potential energy above. Jarzynski showed that this inequality becomes an equality when taking the exponential average of the irreversible work done in taking the system from one state to another: ${ }^{19,37}$

$$
\left\langle e^{-\beta W}\right\rangle=e^{-\beta \Delta G} .
$$

Since this average over the work distribution is exponential, the tails of the distribution carry the most statistical weight. ${ }^{38}$ The samples in these tails are rare, however. This introduces a bias when the tails are not sampled adequately, meaning that this estimator frequently performs poorly.

Convergence is in general expected to be worse in the "deletion" direction than the "insertion" direction, ${ }^{39}$ unless the particular choice of restraints alters this balance. This may seem counter-intuitive since dissipation can be larger when inserting a ghost ligand with the protein in the apo conformation than when deleting the coupled ligand in the holo state (Figure 8, 11a). While dissipation is important, convergence is partly determined by how frequent important realizations are and Jarzynski showed $^{39}$ that important realizations are particularly infrequent in the deletion direction, so that approaches like Widom insertion are practical, though not Widom deletion. Wu and Kofke showed ${ }^{40}$ that transitions in the direction that increases the entropy will not converge with a unidirectional estimator. However, as mentioned above, restraints can alter this balance.

In contrast to the unidirectional Jarzynski equality, the Crooks Fluctuation Theorem $(\mathrm{CFT})^{18,20,41}$ takes transformations in both directions (forward and reverse) into account. This bidirectional approach was shown to converge faster than exponential averaging. ${ }^{42}$ It relates the probability distribution of forward and reverse work values with the free energy difference

$$
\frac{P_{F}(W)}{P_{R}(-W)}=e^{\beta(W-\Delta G)} .
$$

If only unidirectional transformations are performed, the CFT reduces to the Jarzynski equality. The CFT can be solved for $\Delta G$ with the Bennett acceptance ratio method mentioned above (Sec. 2.2). Here, the summation is performed over the number of simulations in 
the forward $\left(n_{f}\right)$ and reverse $\left(n_{r}\right)$ direction:

$$
\sum_{i=1}^{n_{f}} \frac{1}{1+\frac{n_{f}}{n_{r}} e^{\beta\left(W_{f}-\Delta G\right)}}=\sum_{j=1}^{n_{r}} \frac{1}{1+\frac{n_{r}}{n_{f}} e^{-\beta\left(W_{r}-\Delta G\right)}}
$$

The work performed on the system is obtained by accumulating the energy changes as the coupling parameter is changed during the course of the switching transition. ${ }^{43}$

$$
W=\int_{0}^{1} \frac{\delta H(\lambda)}{\delta \lambda} d \lambda
$$

Other uni- and bi-directional estimators are based on a Gaussian approximation of the probability distribution. However, these introduce significant errors even with large amounts of sampling, since the underlying work distributions are not always Gaussian, as demonstrated in the recent SAMPL6 SAMPLing challenge. ${ }^{42}$

Non-equilibrium switching transition data must be collected from independent and uncorrelated trials in order for the average to converge to the true free energy. This is beneficial, in that switching transitions can be run in parallel and thus deploy extremely well in distributed computing environments. This can reduce the wall-clock time of these calculations relative to the EQ approach, at least when a large number of processors is available.

In order to obtain an unbiased estimate of the free energy change, the work distributions should overlap sufficiently (Figure 1). In the case of poor overlap, the CFT result becomes the mean of forward and reverse exponential averages individually computed by the Jarzynski equality. As mentioned above, the exponential average is highly sensitive to the tails of the distribution and therefore, depending on the amount of sampling, a biased estimate. Thus, when overlap is poor, computed free energies are likely incorrect.

\section{Systems}

We compared the performance of the EQ and NEQ methods on two different protein targets, examining two ligands binding to the T4 lysozyme mutant L99A and a ligand binding to HSP90. Each system has its own challenges which we will introduce in this section.

\subsection{The T4 lysozyme mutant L99A is a simple test system, but still has sampling challenges. The T4 lysozyme mutant L99A is} a simple model binding site that has been extensively studied both experimentally and computationally. ${ }^{11,44-49}$ The leucine to alanine mutation leads to the formation of an apolar cavity that binds small molecules. We chose this system for several reasons. First, the dry binding site and the simple, mostly rigid ligands make the system appropriate for the comparison of methods, as sampling times are not excessively long. Secondly, since it is a well studied binding site, it is possible to compare the results with other methods. Lastly, it poses some interesting challenges for alchemical FEC. Toluene is known to bind with two different binding modes (Figure 2a). If the population or free energy of each pose is assessed separately, the crystallographic pose should be the more favorable.

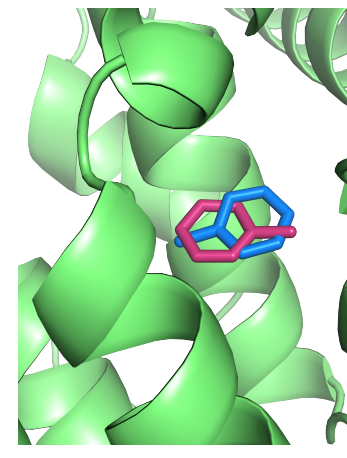

(a)

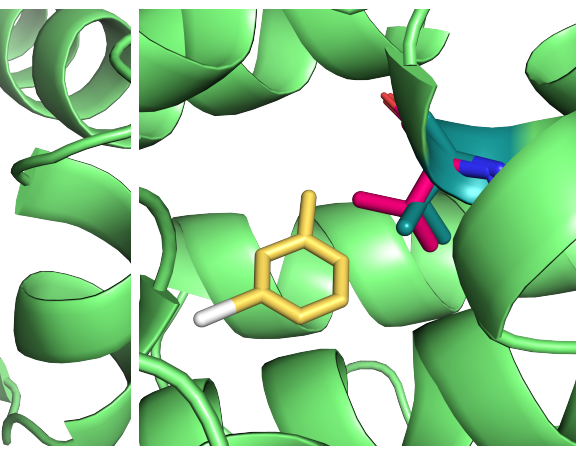

(b)
Figure 2. Toluene and 3-iodotoluene binding to T4 lysozyme mutant L99A. (a) Two binding poses of toluene in T4 lysozyme L99A (green). The crystallographic binding pose (pose I) is shown in blue and the alternative binding mode (pose II) is shown in magenta sticks. (b) Val111 side chain reorientation upon 3 -iodotoluene binding. In the apo state, the Val111 side chain (magenta) points inside the binding pocket. It reorients upon binding (dark green sticks) to make room for 3-iodotoluene (yellow). PDB ID: 4W53

Other ligands, such as 3-iodotoluene and $p$ xylene, induce slow side chain rearrangements upon binding. ${ }^{47}$ In particular, the valine 111 side chain rotates to make room for the slightly larger ligands (Figure 2b). Here, it will be interesting to see if this slow motion can be cap- 
tured in the fast non-equilibrium transitions and whether correct binding free energies can be obtained.

\subsection{HSP90 is a challenging, pharmaceu-} tically relevant target. Even though simple systems, for which convergence can be achieved on reasonable timescales, are highly valuable for method comparison, we are ultimately interested in how the methods perform on pharmaceutically relevant systems. HSP90 is an anticancer drug target, and multiple inhibitors of the protein have been developed. ${ }^{50,51} \mathrm{Compu-}$ tationally, it is a more challenging system than the T4 lysozyme L99A mutant, though still regarded as relatively straightforward on the spectrum of potential pharmaceutical targets. The binding site is solvent exposed and, relative to T4 lysozyme L99A, the ligands are larger, more polar and flexible (Figure 3). While some ligands introduce larger protein conformational changes upon binding, ${ }^{50}$ these are not observed for the ligand considered in this study.

Changes in water placement and occupancy on binding can lead to sampling problems in HSP90. In the unbound state, the solvent completely fills the binding site. In the presence of the ligand, three water molecules are deeply buried in a space between the ligand and the protein (Figure 3). In our MD simulations

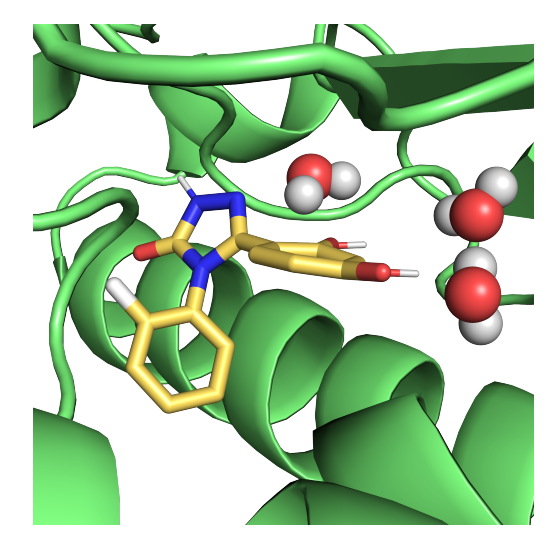

Figure 3. Ligand (yellow) binding to HSP90. Three buried binding side waters are represented as spheres. PDB ID: 5J64

where these water molecules were not included in the starting structure, we found they do not enter the space even after $40 \mathrm{~ns}$ (Sec. 5.5.4). We ran calculations both with and without these bound water molecules to examine their con- tribution to the binding free energy. In the EQ approach, we studied whether the use of HREX alleviates the sampling of this slow motion.

\section{Methods}

\subsection{We constructed a thermodynamic} cycle to calculate the binding free energy.

Ligand binding/unbinding events (associationdissociation) are typically too slow for direct simulation. Therefore, it is more convenient to compute binding free energies via a thermodynamic cycle. Free energy is a state function, meaning that this allows us to construct artificial (more convenient) pathways to get between the states of interest. Figure 4 shows the thermodynamic cycle used in this paper. The binding free energy (panel F) is not simulated directly but obtained through summation along the cycle.

For the decoupling and recoupling of the ligand, we performed both NEQ and EQ calculations. Since these steps are most computationally challenging and expensive, we sought to investigate how NEQ performs compared to EQ.

Some of our simulations use a "bubble-ligand" that repels water to overcome water sampling problems during NEQ switching transitions. Below we show why and how it is used. To incorporate this bubble-ligand into the thermodynamic cycle (right part of Figure 4), we first have to restrain three buried water molecules and a protein loop close to the binding site. This is necessary because the bubble-ligand creates a vacuum in the empty binding site which some side chains of this loop try to fill resulting in conformational changes of the entire loop (Sec. 5.5.3). After restraining the loop, the bubble-ligand is turned on, so that in the next step, where the ligand is decoupled, water cannot enter the binding site. This separates the water sampling problems from the decoupling of the ligand in HSP90.

\subsection{Simulation Details. To prepare} the structures for T4 lysozyme (PDB ID: 4W53) and HSP90 (N-terminal domain, PDB ID: 5J64), first, missing heavy atoms were added with PDBfixer ${ }^{53}$ and protonation states were assigned at $\mathrm{pH} \quad 7$ us- 
(a)

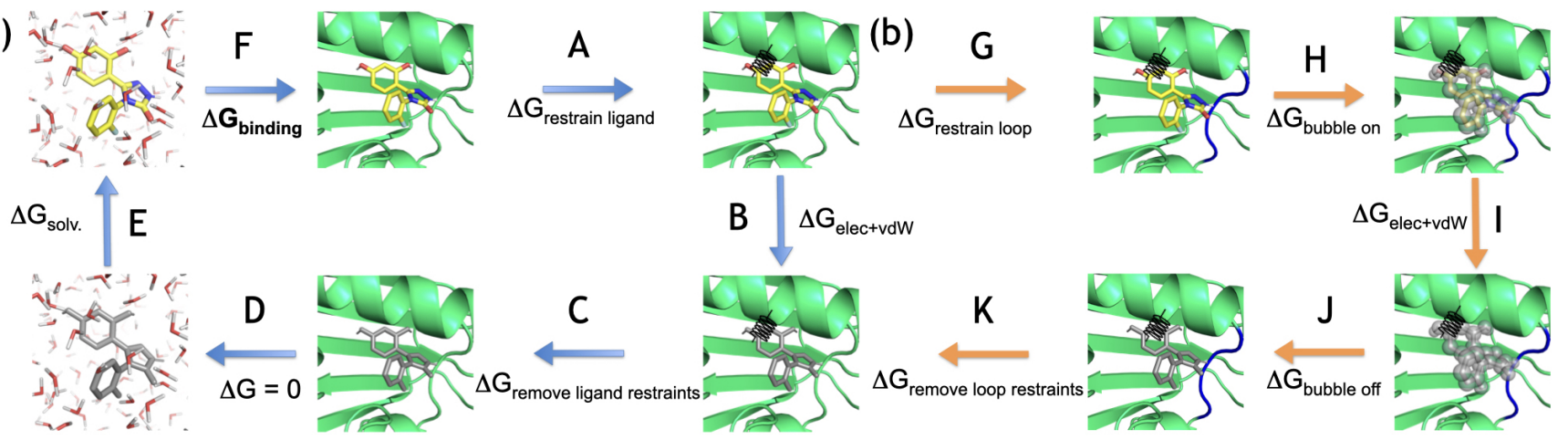

Figure 4. Thermodynamic cycle for computing binding free energies. The left part of the cycle (a) represents the standard cycle used in this paper, the right part (b) was only added for calculations including a bubble-ligand (Sec. 5.5.3). The ligand is restrained using orientational (Boresch-style) ${ }^{52}$ restraints (A) and then decoupled in the binding site, meaning that the intermolecular electrostatic and van der Waals (vdW) interactions of the ligand are turned off $(\mathrm{B})$. The restraints are released analytically $(\mathrm{C})$, the ligand is transferred from the complex into the solvent (D) and then coupled in the solvent (E). The next leg (F) represents the free energy difference upon ligand binding which is obtained through summation along the cycle. (b) For calculations that include a bubble-ligand to overcome insufficiencies in water sampling, a loop and three buried water molecules are restrained (G, Sec. 5.5.3), then the $\mathrm{vdW}$ interactions between the bubble-ligand and water are turned on $(\mathrm{H})$. In the next step the ligand is decoupled in the binding site (I) and the bubble-ligand prevents water from entering the site. The bubble-ligand is then turned off again $(\mathrm{J})$, the restraints on the loop and buried water molecules are released $(\mathrm{K})$ which leads back to the regular cycle.

ing the PDB2PQR server. ${ }^{54,55}$ ParmEd (http://parmed.github.io/ParmEd) was used to convert the pqr output to a pdb file. For the ligands, OpenEye toolkits ${ }^{56}$ were used to add missing hydrogen atoms and to check the bond order and connectivities. For toluene and the HSP90 ligand the binding mode was extracted from the crystal structure. An alternative binding mode of toluene and the binding mode of 3-iodotoluene were obtained from the supporting information of the BLUES paper. ${ }^{57}$

The systems of the protein-ligand complex and the ligand in solution were then generated using YANK. ${ }^{58}$ The protein was parameterized with Amberff14SB ${ }^{59}$ and the ligands with GAFF2 ${ }^{60}$ and AM1-BCC charges. ${ }^{61}$ The protein-ligand complex was solvated with TIP3P water ${ }^{62}$ in a cubic box and $\mathrm{Na}^{+}$and $\mathrm{Cl}^{-}$ions were added until a salt concentration of $50 \mathrm{mM}$ (T4 lysozyme) or $150 \mathrm{mM}$ (HSP90) was reached. The Amber output files of the system were then converted to GROMACS structure and topology files using ParmEd. For HSP90 two protein-ligand systems were prepared, one where all water molecules from the crystal structure were removed, while in the second crystallographic waters in the binding site were retained. The topology and coordinate files are available in the supporting information (SI).

Orientational (Boresch-style) restraints were applied by restraining 3 atoms in the protein and 3 atoms in the ligand through one distance, two angle and 3 dihedral restraints with a force constant of $20 \mathrm{kcal} /\left(\mathrm{mol} * \AA^{2}\right)$ for the bond and $20 \mathrm{kcal} /\left(\mathrm{mol} * \mathrm{rad}^{2}\right)$ for angles and dihedrals. A table of the protein and ligand atoms used to restrain the complex can be found in SI Table S2. The atoms were selected during the setup of the system with YANK. YANK's selection process picked heavy atoms that are not co-linear and ensured that the distance restraint involved a protein-ligand atom pair within 1-4 A. Previously, the calculated binding free energy was shown ${ }^{52,63}$ to be independent of the atom selection and force constants. Here, the restraint contribution (Figure 4 panel A) was calculated by running two equilibrium states, one of the coupled, restrained ligand and one of the coupled ligand without restraints for $10 \mathrm{~ns}$ each. The free energy difference was estimated using BAR and three independent replicates were run to check for convergence and to estimate the uncertainty. 
All MD simulations were performed using GROMACS 2018.3. ${ }^{64,65}$ In the EQ approach, every $\lambda$ window was energy minimized using steepest descent for 5000 steps, then equilibrated in the canonical ensemble for 10 ps at 298.15 K. Afterwards, the system was equilibrated in the isothermal-isobaric ensemble for 100 ps at a pressure of 1 bar. The production run was performed at constant NPT as well. All MD simulations were performed using the leap-frog integrator at a timestep of 2 fs. In the alchemical pathway, first the electrostatic interactions were decoupled, then the sterics, applying a soft-core potential. ${ }^{66}$ The soft-core potential is used to avoid instabilities in intermediate lambda windows. Although new softcore potential functions, including the smoothstep softcore potentials, ${ }^{67}$ have been developed recently, the soft-core potential from Beutler et al. ${ }^{66}$ was used in this work as this is widely available in GROMACS. EQ and NEQ methods were treated equivalently with respect to the soft-core potential, thus both approaches were evaluated at equivalent conditions. The intramolecular interactions of the ligand were not changed during the process. Complete settings can be found in the mdp files provided in the SI. Although the convergence rate can be different among $\lambda$ windows, ${ }^{34-36}$ we ran all alchemical states for an equal amount of time for convenience. The overall convergence of the free energy difference will therefore be determined by the slowest converging window.

For the NEQ approach, end state simulations were performed in the interacting state (state A) and the decoupled state (state B) using the same general procedure (minimization and equilibration) as in the EQ approach. Then, either the first nanosecond (in the case of toluene) or the first $5 \mathrm{~ns}$ of the production run was discarded for equilibration and frames extracted every 40 ps (T4 lysozyme) or every 50 ps (HSP90). Fast switching transitions from state $\mathrm{A}$ to $\mathrm{B}(\mathrm{A} 2 \mathrm{~B})$ and vice versa $(\mathrm{B} 2 \mathrm{~A})$ were performed where the $\Delta \lambda=1 / n_{\text {steps }}$ where $n_{\text {steps }}$ is the number of steps. Since electrostatics and sterics were switched simultaneously, a softcore potential was applied for both non-bonded interactions with the soft-core parameter sc- alpha $=0.5$, sc-power $=1$ and sc-sigma $=0.3$, as is typical.

Some of the simulations included a bubbleligand which overlaps with the normal ligand but interacts only with water molecules. The bubble-ligand interacts with the water (not including the three buried water molecules) through Lennard-Jones interactions while not interacting with any other molecules in the system, and is designed (Sec. 5.5.3) to alleviate water sampling problems in the binding site at intermediate lambda values. The Lennard-Jones parameters $\sigma$ and $\epsilon$ of this bubble-ligand were set to $0.33 \mathrm{~nm}$ and $0.41 \mathrm{~kJ} / \mathrm{mol}$ for all atoms of the bubble-ligand (see topology file SI).

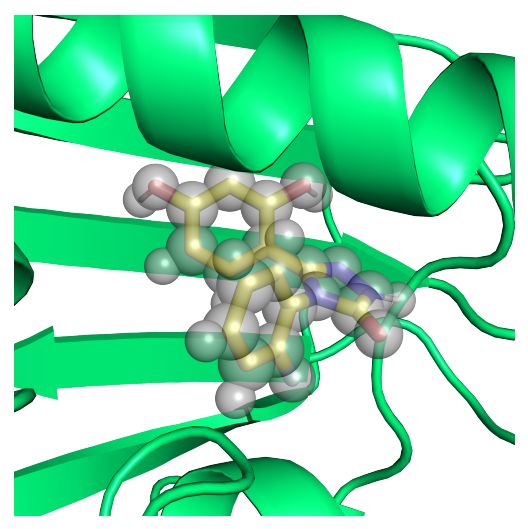

Figure 5. The bubble-ligand sits on top of the normal HSP90 ligand, but only interacts with water molecules in order to separate water sampling problems from the decoupling of the ligand. The bubble ligand is shown in grey spheres and the real ligand in colorized sticks.

We also introduced position restraints to prevent the side chains of a nearby protein loop from filling the vacuum of the empty binding site created by the bubble-ligand. The restraints were applied to the side chain backbone atoms using a force constant of $1000 \mathrm{~kJ} /(\mathrm{mol} *$ $\left.n m^{2}\right)$. The three buried water molecules which do not interact with the bubble-ligand were restrained with position restraints as well. The restraints were turned on and off using $15 \lambda$ windows with varying force constants.

\subsection{We obtained uncertainty estimates} by running independent repeats. In order to obtain reliable error estimates, we performed independent replicates, both for the EQ and the NEQ approach. Given that MD simulations are sensitive to the initial conditions, two 
trajectories from the same system can diverge quickly. The divergence of an ensemble of independent simulations from various starting conditions provides insight into sensitivity to initial conditions. Although in the NEQ approach the fast-switching transitions are already independent of one another (at least to the extent that the equilibrium snapshots from which they are started are assumed uncorrelated), we also ran repeats of the end state simulations. Extracting snapshots from independent shorter MD simulations rather than one larger one can ensure better sampling of phase space. Bootstrapping of NEQ work values was used for the individual replicates but we found that it underestimated the uncertainty.

For the NEQ approach, analytical error estimates can help detect lack of overlap of the work distributions. For maximum likelihood methods, like BAR, the variance asymptotically converges to the inverse of the Fisher information of the joint distribution of, in this case, forward and reverse work values. ${ }^{68}$ Using this variance estimate, the analytical uncertainty can be vastly overestimated when overlap is poor (Sec. 5.5 and especially Figure S15). We therefore only use this method as a diagnostic metric in the NEQ approach. A high uncertainly estimate indicates a potential overlap problem and that results cannot be trusted.

4.4. Overlapping work distributions don't guarantee reliable free energy estimates. Overlapping work distributions are a necessary but not sufficient requirement to obtain reliable free energy estimates. Consider for example a system where slow side chain rearrangements occur upon ligand binding. If this rearrangement is neither sampled in the equilibrium end state simulation nor in the fast switching transitions, the work distributions might overlap well. Computed free energies, however will not be correct, because the contribution of structural rearrangement in the binding site is not accounted for (Sec. 5.3.2, Figure 11). Running independent replicates helped assess these issues in this work. Phase space overlap measurements can serve as a diagnostic metric and have been examined fairly extensively for equilibrium calculations. ${ }^{14,69}$ Even though sufficient overlap of the potential energy distributions of neighboring $\lambda$ states is crucial to obtain an unbiased estimate, it is important to remember that overlap alone does not guarantee that sampling is adequate.

4.5. We compared the EQ and NEQ approaches based on convergence of computed free energy differences where possible. Since the two approaches start their simulations from systems parameterized and prepared in the same way, the calculated free energy differences should converge to the same value when sampling is sufficient. In order to assess which method converges faster, we looked at how the cumulative $\Delta G$, averaged over trials, changes over time.

A method can be considered efficient if it quickly reduces the standard deviation and bias. In the cases where convergence was achieved we therefore investigated how standard deviation and bias behave over the observed simulation time scale for the two methods. For the bias we used the converged final $\Delta G$ estimate as the true reference, although the supposedly converged free energy difference might not be correct if the most important conformations have not been sampled.

We had originally hoped to compare efficiency of EQ and NEQ approaches on all the systems considered here, but to assess efficiency, we must obtain well converged free energy estimates. However, in some of our simulations convergence is not achieved due to sampling problems we explain elsewhere (Sec. 5.), making it impossible to compare the efficiency of the two approaches.

In all systems, we used the MBAR estimator as implemented in alchemlyb/pymbar 28,70 to estimate free energy differences in the EQ approach and computed then the mean and standard deviation of three independent repeats.

For the NEQ approach we calculated free energy differences using the CFT/BAR method as implemented in pmx. ${ }^{71,72}$ Instead of calculating the mean of three independent replicates, we pooled together all work values from the repeats to estimate one free energy difference. For the uncertainty estimate, we calculated the standard deviation obtained by treating the in- 
dividual repeats separately. This analysis was performed for decoupling the ligand in the binding site as well as coupling it in the solvent.

\subsection{We assessed potential sampling lim-}

itations. To help assess sampling problems in this work, we find it helpful to examine several properties relating to free energies, as we will further discuss in Sec. 5. Particularly, we compute the Pearson correlation coefficient between the work values and all side chain $(\xi)$ dihedral angles in the protein using the SciPy package in Python, with $\xi$ angles calculated via GROMACS. Additionally, we examine $\mathrm{dH} / \mathrm{d} \lambda$ values and their relationship to other degrees of freedom. Here, we do not use $\mathrm{dH} / \mathrm{d} \lambda$ values for free energy estimates, but they can be used to do so via TI, and these $\mathrm{dH} / \mathrm{d} \lambda$ values provide a view into the potential contributions of specific motions in specific trajectories to binding free energies (since they are measured for individual snapshots, allowing us to identify rearrangements/motions which lead to sudden changes in $\mathrm{dH} / \mathrm{d} \lambda$ ). We find (Sec. 5.3 and 5.5) that these are a helpful diagnostic.

\section{Results}

In this study we compare equilibrium and nonequilibrium approaches to calculating binding free energies of ligands to two protein targets, T4 lysozyme L99A and HSP90, and examine the different challenges these approaches encounter for the systems studied.

We first compare the performance of the two approaches for calculation of hydration free energies of the three ligands, which were computed as part of the thermodynamic cycle (Figure 4E). Those calculations converged well enough to compare the efficiency of the methods. After examining hydration free energies, we focus on protein-ligand systems.

\subsection{Comparing the efficiency of EQ and NEQ on the hydration free energy.} The EQ and NEQ approach yielded similar results for the hydration free energy of toluene, 3-iodotoluene and the HSP90 ligand. In Supporting information (SI) Figure S1, we show how the cumulative free energy difference, the standard deviation and the bias converged for the two methods.
Using the same amount of sampling, the two approaches converged within uncertainty to the same values for toluene and 3-iodotoluene. The methods agreed to within $0.2 \mathrm{kcal} / \mathrm{mol}$ of each other fairly quickly ( $\sim 40$ ns total simulation time). In the case of the HSP90 compound, it took $\sim 200$ ns to agree within $0.2 \mathrm{kcal} / \mathrm{mol}$, and EQ and NEQ free energy estimates did not converge to statistically the same result, but were very close $(\Delta \Delta \mathrm{G}=0.14 \mathrm{kcal} / \mathrm{mol})$. When running longer (500 ps) but fewer NEQ switching transitions the methods agreed, but the standard deviation was slightly higher (SI Figure S1). For these three ligands, the NEQ approach was able to reduce the standard deviation and the bias faster than the EQ approach, suggesting that it is more efficient. However, this needs investigation on a larger set of molecules.

\subsection{Toluene binding to T4 lysozyme} mutant L99A. With the force field used here, toluene binds to the cavity in this T4 lysozyme mutant with two (non-symmetric) binding modes (Figure 2). The crystallographic binding pose (pose I) has a more favorable binding free energy than the other pose (pose II) and interconversion between these two binding modes is very slow compared to typical simulation timescales (e.g. $100 \mathrm{~ns}$ or more). ${ }^{57}$ Therefore we ran separate calculations for each pose and applied restraints to keep the ligand from transitioning between binding modes (SI Figure S2).

While all the protocols we report used the same amount of overall sampling time, we investigated the effect of the transition length versus number of switching transitions on the $\Delta G$ estimate in the NEQ calculations. For both binding poses, the EQ and NEQ approaches converged to a statistically equivalent value of the free energy difference (Figure 6). The standard deviation in pose I, however, was higher with the non-equilibrium method for all three transition lengths.

Finally, the overall binding free energy of toluene for both poses was obtained through the thermodynamic cycle (Table 1, results of individual legs of the cycle in SI Table S3). A symmetry correction of $-k_{B} T \ln 2$ 

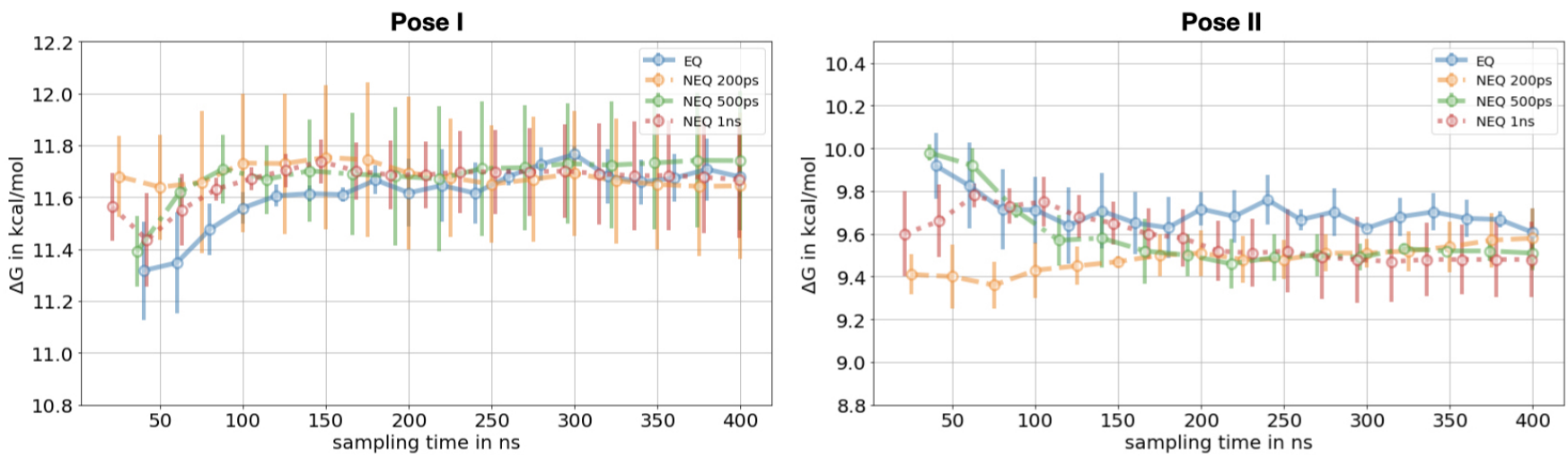

Figure 6. Decoupling of toluene in the binding site. The free energy difference for the EQ and NEQ approach for both poses is shown as a function of the sampling time. For the EQ approach we show the mean free energy difference across three replicates while for the NEQ approach the free energy estimate was obtained by pooling work values from three replicates and estimating one free energy difference using BAR (Sec. 4.5). The uncertainty estimate in both approaches is the standard deviation across three replicates. The NEQ protocols differ in the lengths of the NEQ switching transitions, which are $200 \mathrm{ps}, 500 \mathrm{ps}$ and $1 \mathrm{~ns}$, respectively. The number of switching transitions differs among the protocols in such a way that the same amount of overall sampling time is used in all protocols. All protocols converged to the same free energy difference within uncertainty.

$\approx-0.4 \mathrm{kcal} / \mathrm{mol}$ was added to account for the symmetry-equivalent binding mode. ${ }^{63}$ The crystallographic pose was correctly predicted with a more negative $\Delta G$ with both methods (Table 1). While the binding free energy for the non-crystallographic binding mode is not an experimental observable, when combined with the binding free energy for the crystallographic pose, it tells us the relative population of the two binding modes.

Table 1. Binding free energy $\Delta G^{\circ}$ for the toluene/T4 lysozyme system.

\begin{tabular}{|l|l|l|l|}
\hline & $\begin{array}{l}\text { EQ } \\
{[\mathrm{kcal} / \mathrm{mol}]}\end{array}$ & $\begin{array}{l}\text { NEQ } \\
{[\mathrm{kcal} / \mathrm{mol}]}\end{array}$ & $\begin{array}{l}\text { experimental } \\
{[\mathrm{kcal} / \mathrm{mol}]}\end{array}$ \\
\hline \hline $\begin{array}{l}\text { pose I } \\
\begin{array}{l}\text { crystallo- } \\
\text { graphic pose) }\end{array}\end{array}$ & $-4.6 \pm 0.1$ & $-4.7 \pm 0.3$ & $-5.52 \pm 0.04$ \\
\hline pose II & $-3.7 \pm 0.1$ & $-3.7 \pm 0.1$ & \\
\hline
\end{tabular}

\subsubsection{Isoleucine 78 reorients upon bind-} ing of toluene. The side chain of isoleucine 78 (Ile78) reorients upon binding of toluene, causing problems for both the EQ and NEQ approaches if this reorientation is not sampled adequately. While the side chain showed partial occupancy of both rotamers in the noninteracting state (state B), Ile78 had one preferred orientation in the bound state (state A, Figure 7).

The reorientation of the Ile78 dihedral angle

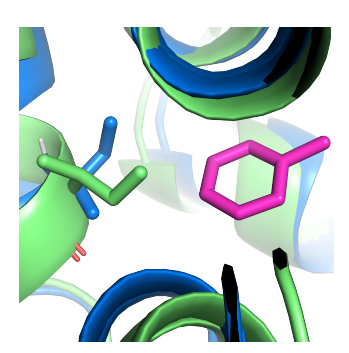

(a)

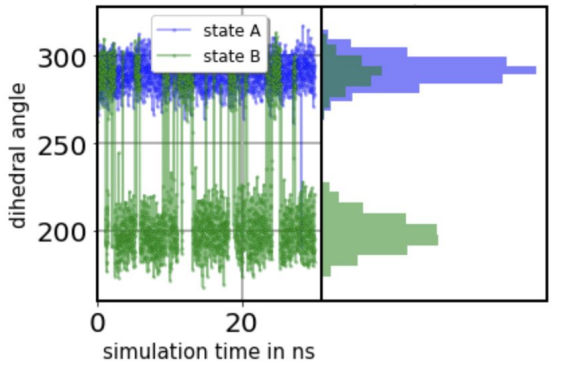

(b)
Figure 7. The side chain Ile78 reoriented upon binding of toluene to T4 lysozyme L99A. (a) In the bound state the Ile78 side chain occupied one rotamer state (blue sticks), while the side chain showed partial occupancy of two rotamers (blue and green sticks) in the unbound state. (b) The $\xi 1$ dihedral angle of Ile78 as a function of simulation time in the simulation of the interacting (state A, blue) and the noninteracting state (state B, green) and its distribution. The non-interacting state had to be sampled long enough to observe multiple transitions between the rotamers.

occurred relatively fast on timescales of a few hundred ps - which is faster than simulation timescales and we seemed to be able to adequately sample the motion here (Figure 6 and S3). This helped us develop techniques to better assess important protein motion.

\subsubsection{Side chain reorientation in NEQ} switching transitions. In the NEQ approach, end states have to be sampled long enough to start transitions from the correct distribution 
of dihedral angles and/or the transitions have to be slow enough to allow for reorientation (if needed) on ligand binding, otherwise the final distribution of dihedral angles will closely match the starting distribution.

According to the Crooks fluctuation theorem, provided that transitions are started from the correct distribution of conformational states in the end states and that a sufficient number of transitions are performed, the method will converge to the correct free energy difference. This is true even if the final distribution of dihedral angles after the transitions does not match the equilibrium distribution in that end state.

As can be seen in SI Figure S3, fast transitions (200 ps) can be long enough to allow for the side chain to reorient, however, the rotamer populations do not resemble those after the transition as observed in a long end state simulation. This does not mean that resulting values are necessarily wrong as they would be if the dominant rotamer were never sampled in the non-equilibrium switches (Sec. 5.3). Here, running shorter transitions, where we sampled the rotamer switching only sometimes, resulted in free energy estimates that were statistically equivalent to those obtained using longer transitions, in which the dominant rotamer was sampled most of the time, presumably because the free energy estimator gives the most weight to those transitions which discover the favorable rotamer. The standard deviation was also comparable among different NEQ protocols (Figure 6). This shows that sampling the side chain reorientation in some switching transitions is sufficient. The standard deviation between independent replicates for pose I is higher with the NEQ approach than with the EQ approach for all transitions lengths $(0.2-0.3 \mathrm{kcal} / \mathrm{mol} \mathrm{vs}$. $0.1 \mathrm{kcal} / \mathrm{mol}$ ), suggesting that the protein undergoes additional conformational changes.

In this system, sampling of different rotamers of Ile78 led to a bimodal work distribution in the direction of restoring the ligand-protein interactions (the direction we call B2A, as our $\mathrm{B}$ state is the noninteracting state), and this was especially common for fast transitions (Figure 8). While a bimodal distribution of work values itself does not necessarily pose a prob-

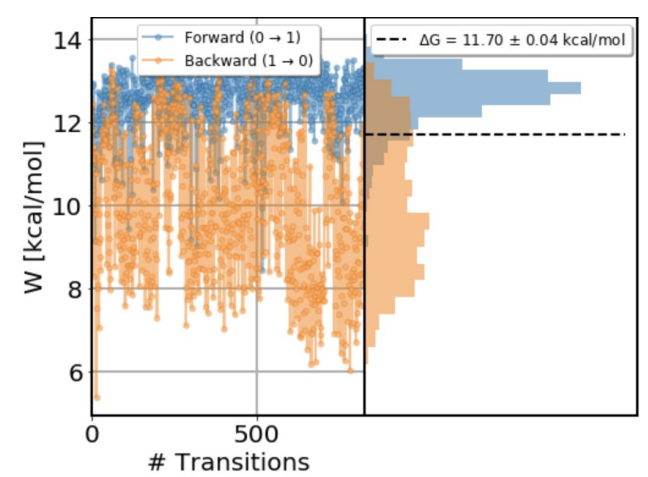

Figure 8. Work values for toluene binding to T4 lysozyme L99A, in forward (blue) and reverse (orange) direction. Shown are values measured for each attempted transition, as well as the distribution of the work values. The transition length for each transition was 200 ps. Data from trials in the reverse direction shows sudden changes in work values and the distribution of work values is bimodal indicating a slow degree of freedom in the system.

lem, it can pose a problem when it results from drawing from an incorrect or biased distribution of states at the end states - such as when a slow conformational transition is inadequately sampled for one or both end states - or when it results from transitions which are too fast to sample an important conformational change.

Concretely, for example, consider a situation where a minor rotameric state for a particular sidechain ought to be slightly populated in the bound state at equilibrium and becomes dominant in the unbound state. If the actual sampling of the bound state misses that minor conformer entirely and switching to the unbound state is so rapid as to miss rotameric transitions, there is a high likelihood that resulting free energy estimates will be biased (unless sampling of reverse transformations is somehow sufficient to recover). Here, then, we find it helpful to assess dihedral sampling in the end states and assess whether switching transitions ever sample rotamers appropriate for their target end state.

If the rotamer population is not sampled correctly in the end states, the results will be inaccurate or imprecise, and a bimodal distribution can in some cases be a warning sign that sampling might be complex or slow. Restraining the Ile78 side chain resulted in a unimodal work distribution (SI Figure S6) which further supports our findings that different rotamers of 
Ile78 in the B state led to a bimodal work distribution in the reverse transitions. The situation could have been worse if the noninteracting state did not already spend time sampling both rotamers and if the reorientation occurred on longer timescales. If a conformational transition is truly needed on binding then instead of having a bimodal distribution the final distribution might be unimodal, but wrong, as discussed in Sec. 4.4 and in Sec. 5.3.2.

There are generally two scenarios that can lead to dissipation and non-overlapping work distributions (SI Figure S9, S15) or bimodal work distributions (Figure 8): In the first scenario the system ends up in the correct end state but during NEQ switches was driven rapidly and accordingly heat was dissipated (friction). Secondly, the system does not end up in the equilibrium end state at the end of the short transition. An example for this second scenario is the Ile78 side chain orientation discussed above.

To investigate whether the Boresch-style restraints were a source for the large dissipation seen in the reverse process (orange work distribution Figure 8), we performed calculations applying a lower force constant on the orientational (Boresch-style) restraints (see SI Figure S4). The average dissipated work $W_{d}$, where $W_{d}=W^{R}+\Delta G=W^{F}-\Delta G,{ }^{39}$ was the same for both protocols $(1.7 \mathrm{kcal} / \mathrm{mol}$ in the reverse direction and $0.9 \mathrm{kcal} / \mathrm{mol}$ in the forward direction).

Center of mass (COM) - COM flat-bottom harmonic distance restraints are an alternative to orientational restraints and have been applied to binding free energy calculations. ${ }^{73} \mathrm{We}$ performed calculations restraining the distance between the COM of the ligand and the COM of a protein side chain and compared the results of the two restraint approaches (see SI Table S4). The dissipated work was higher in the COM-COM restraining protocol $(3.8 \mathrm{kcal} / \mathrm{mol}$ in the reverse direction and $1.4 \mathrm{kcal} / \mathrm{mol}$ in the forward direction) compared to the one using Boresch-style restraints. With a single COMCOM distance restraint, the ligand can sample the sphere with the radius of that distance restraint which presumably caused the larger dissipation. These findings suggests that the Boresch-style restraints were not a major source for the dissipation seen in Figure 8.

5.2.3. The Ile78 orientation correlated with the work values as detected with the Pearson correlation coefficient. In the NEQ approach, we detected slow protein motions by calculating the Pearson correlation coefficient (PCC) between the work values and all side chain dihedral angles in the protein. We calculated the dihedral angles from the set of first frames and the set of last frames of NEQ switching transitions. Then, we took the work values for the set of forward transitions and correlated it with the set of initial dihedral angles; we also did the same for the set of final dihedral angles. This gave two separate PCC for each dihedral angle (one using the dihedral angles from the first frames and one the dihedral angle of the set of last frames). We performed the calculation in both the forward and reverse direction (decoupling and coupling). When the ligand appeared in the binding site (backward direction), a high PCC was found between the $\xi 1$ dihedral angle of Ile78 and the reverse work values in both poses. The correlation was highest in the NEQ protocol with 200 ps/transitions using the structure of the first frame of the switching transitions ( $\mathrm{PCC}=0.5-0.6)$, as can be seen in Figure 9a. The PCC was also high using the last frame of the transitions (0.5-0.6), suggesting that it is important to sample the side chain reorientation at least in some transitions. The PCC decreases as the transition length increases, especially the correlation between work and the starting orientation (first frame of transitions)(mostly $\mathrm{PCC}<0.5)$, since the side chain reorients more often in longer switching transitions. Figure 9b shows an example of a slow motion that is not correlated with work values. Asp127 is not close to the binding site and although the motion is slow, it does not seem to be important.

Calculating the correlation between work values and dihedral angles in the protein may be a useful general approach for identifying potentially problematic degrees of freedom. This approach will work best when the number of such degrees of freedom is low. If multiple side chains 


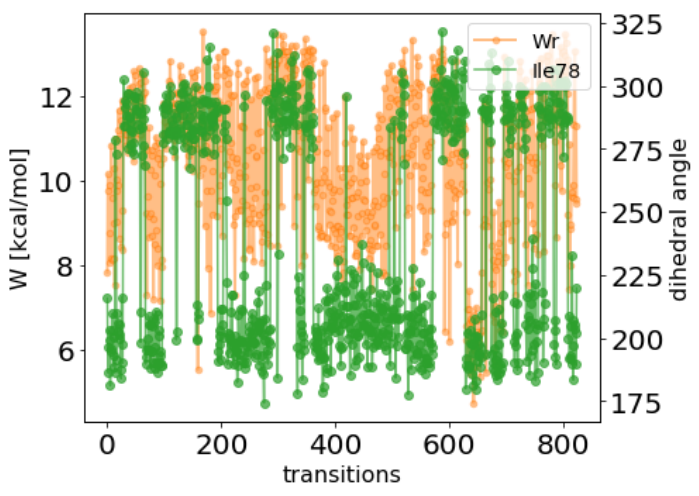

(a)

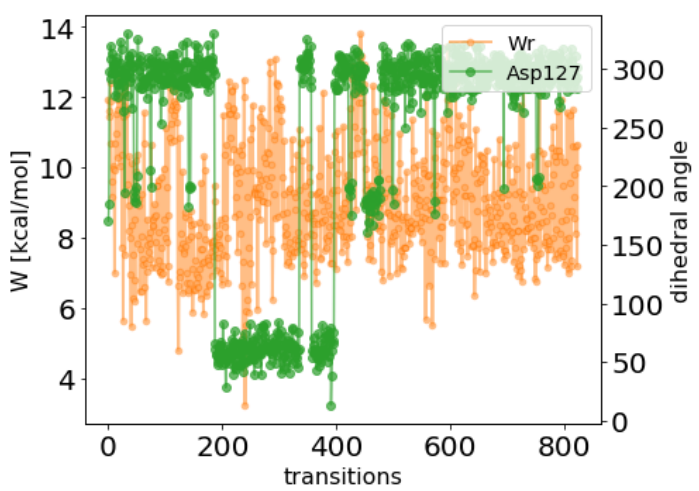

(b)

Figure 9. Correlation between reverse work values (Wr) and the dihedral angle of Ile78 (a) and Asp127 (b) for toluene binding to T4 lysozyme L99A. The work values (orange) and the dihedral angles (green) are plotted as a function of the transition number. The dihedral angle was calculated from the set of first frames of the NEQ transitions. (a) Work values are correlated with the dihedral angle of Ile78, which is supported by a high Pearson correlation coefficient $(\mathrm{PCC}=0.5)$. (b) A low PCC of 0.04 likely indicates no significant correlation between dihedral angle Asp127 and work values. Asp127 was included as an example of a motion that is not correlated with the free energy difference. Even though the motion is slow, it appears not to be important.

reorient upon binding, the correlation between the work and the orientation of each individual side chain will be reduced, making it more difficult to identify slow motions. Furthermore, the metric is prone to false positives due to accidental correlations that can cross significance testing thresholds, as illustrated in this comic. ${ }^{74}$ If 200 residues are predicted not to correlate with the work values, a $5 \%$ false positive rate will mean that there are 10 false correlations. Careful inspection (e.g. considering proximity to the binding site) and cross-validation with replicates can help ensure apparent correlations are not spurious.

However, it is important to note that NEQ free energy calculations do not require switching transitions to sample all relevant conformational states; what is required is that end state sampling populate the correct equilibrium distribution of states, and switching transitions must be sufficiently numerous to provide an adequate average over possible work values. Thus, the presence of work values that correlate with a particular slow conformational degree of freedom does not necessarily indicate sampling problems; rather, it means that practitioners need to check that the end states in fact sample the correct equilibrium distribution of states. If they do not, results will be inaccurate or at least imprecise.

\subsubsection{In the equilibrium FEC, the Ile78 orientation correlates with $\mathrm{dH} / \mathrm{d} \lambda$ val-} ues. In the EQ approach, sampling problems can in some cases be detected by examining sudden changes in the $\mathrm{dH} / \mathrm{d} \lambda$ values and identifying related structural degrees of freedom. We plotted the running average of the $\mathrm{dH} / \mathrm{d} \lambda$ values over the simulation time for every state, picked a $\lambda$ window where there was an obvious jump in the $\mathrm{dH} / \mathrm{d} \lambda$ value and looked at frames from the trajectory near that jump. Once any potential sampling problem - such as a motion which might be responsible for the sudden jump in $\mathrm{dH} / \mathrm{d} \lambda$ - was identified we correlated the motion with the $\mathrm{dH} / \mathrm{d} \lambda$ curves of all $\lambda$ windows to assess correlations between that structural degree of freedom and $\mathrm{dH} / \mathrm{d} \lambda$ values.

Here, we find that sampling problems primarily occurred in the alchemical intermediate states. In the EQ approach, each state has to be sampled at equilibrium, meaning that the correct equilibrium distribution of the Ile78 dihedral angle has to be sampled at every $\lambda$ window. This can be especially challenging in intermediate states where energy barriers between the rotamers can be high. In SI Figure S5 we show an example of the correlation between the $\mathrm{dH} / \mathrm{d} \lambda$ values in one $\lambda$ window and the dihedral angle of Ile78. The three replicates did not sample the same rotamer distribution of the side chain indicating that sampling time was not sufficient 
to reach equilibrium.

This approach of correlating $\mathrm{dH} / \mathrm{d} \lambda$ values with structural degrees of freedom can be a useful general strategy for identifying potentially problematic degrees of freedom in equilibrium FEC. This method is comparable to examining sudden changes in work values in the NEQ approach in that it helps to narrow down a large amount of trajectory data to a specific set of frames in which a slow but important rearrangement may occur.

Here, independent replicates converged well (Figure 6) suggesting that the motion of the Ile78 rearrangement, although not sampled adequately in every alchemical state, was overall sampled well enough to only have a minor impact on the free energy difference.

\subsection{3-Iodotoluene binding to $\mathrm{T} 4$} lysozyme mutant L99A. For 3-iodotoluene binding to T4 lysozyme (L99A), in both EQ and NEQ calculations the independent replicates did not converge to the same result in 400 ns total simulation time, indicating inadequate sampling. In Figure 10 we show the mean and standard deviation for the independent repeats of the EQ, and different protocols of the NEQ approach; for details on the individual repeats see SI Figures S7 and S8. The standard deviation $\sigma$ for the EQ approach was higher than in the toluene case $\left(\sigma_{3 \text {-iodotoluene }}=0.4 \mathrm{kcal} / \mathrm{mol}\right.$, $\left.\sigma_{\text {toluene }}=0.1 \mathrm{kcal} / \mathrm{mol}\right)$ suggesting that sampling problems were more severe. For the NEQ protocols, which all used the same total simulation time, the $\Delta G$ estimate depended on the length of the NEQ switching transitions. Since overlap of the work distributions was poor, even with $4 \mathrm{~ns}$ /transition (SI Figure S9), results are likely not to be trusted. The overall binding free energy of 3-iodotoluene, which was obtained through the thermodynamic cycle, varies between -4.8 and $-5.7 \mathrm{kcal} / \mathrm{mol}$ among different protocols, as shown in SI Table S5. The experimental binding affinity of 3-iodotoluene to T4 lysozyme L99A was not found in the literature. Here our focus is not on agreement with experimental values, since this is also a function of the force field and other factors; rather, we focus on whether we have obtained converged values.

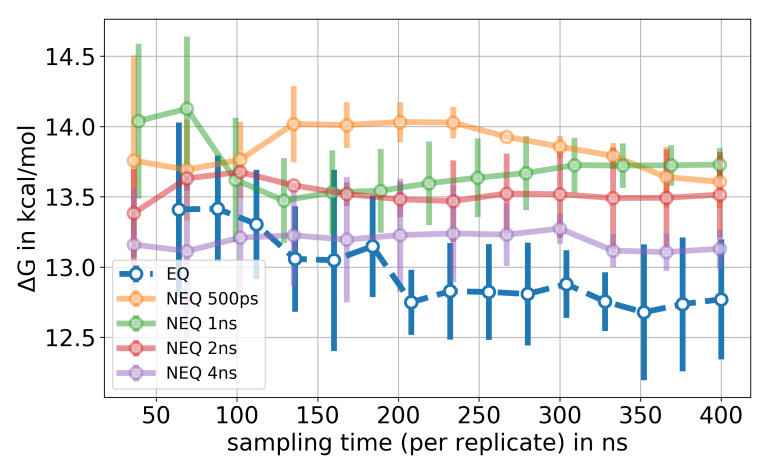

Figure 10. Free energy difference of decoupling 3iodotoluene in the binding site as a function of sampling time. For the EQ approach we show the mean free energy difference across three replicates while for the NEQ approach the free energy estimate was obtained by pooling work values from three replicates and estimating one free energy difference using BAR (Sec. 4.5). The uncertainty estimate in both approaches is the standard deviation across three replicates. Same amount of total simulation time was used for the EQ and the four protocols of the NEQ approach. The different NEQ protocols differ in the length and the number of NEQ transitions. The standard deviation, especially for the EQ approach, was high and the $\Delta G$ estimate of the NEQ protocols depended on the transition length, suggesting insufficiencies in sampling.

\subsubsection{Valine 111 reorients upon bind-} ing of 3 -iodotoluene. Slow conformational changes in the protein prevent convergence in both methods on the timescale of our simulation. 3-Iodotoluene, being more bulky than toluene, induces a rearrangement of the valine 111 (Val111) side chain upon binding (Figure $2 \mathrm{~b})$. The timescale for this reorientation is relatively slow (a couple of nanoseconds) and consequently results are highly sensitive to the initial protein conformation (Figure 11). The predicted free energy change is either too favorable or too unfavorable depending on which structure was used as a starting point. If this conformational change is not sampled properly in the free energy calculation, running simulations starting in the apo protein structure results in a predicted free energy which is too unfavorable. The contribution of protein rearrangement upon binding and potential steric clashes with the protein are not accounted for in this scenario. On the other hand, the free energy change is predicted as too favorable when simu- 
lations are started in the holo protein structure. The protein is deformed upon binding and the energetic cost of this protein strain energy is missing. ${ }^{47}$

\subsubsection{This side chain rearrangement is} not sampled adequately in NEQ and EQ calculations. In the NEQ approach it is important to sample the correct orientation of Val111 in the end states at equilibrium and to run the transitions between the end states slowly enough to allow for a rearrangement at least in some transitions (as discussed in Sec. 5.2.2). Although the free energy estimate should be independent of the switching rate, provided that a sufficient number of work values was collected, we found that the efficiency of a protocol can dependent on the switching rate. Performing the transitions at a slower rate increases the probability of observing the reorientation of a side chain, because the transition has less dissipation and follows closer the free energy gradient. Here, dissipation was large, and reducing the switching rate can be more efficient than running faster transitions (see Figure 10) which would require a longer total simulation time. Simulations were started in the bound protein conformation. The end state simulation of the decoupled state has therefore be run long enough so that the Val111 side chain can reorient adequately to obtain a correct equilibrium distribution of conformations. Here, the alternate rotamer (after reorientation) should be dominant in the unbound ensemble, so we discard all frames before reorientation to equilibration. This would not be necessary if the end state simulation was run long enough to capture the equilibrium distribution of conformations instead of being biased by the starting configuration of Val111.

Starting NEQ transitions from structures where Val111 had not reoriented yet led falsely to good overlap of the work distributions but a wrong estimate of the free energy difference (Figure 11). Only a few transitions starting from the "wrong" structure (the overpopulated minor rotamer) had a huge impact on the result since the tails of the distribution have a large statistical weight for the $\Delta G$ estimate. When as few as 17 transitions starting from the wrong
Val111 rotamer were included (Figure 11c, discard 60), the free energy did not converge to the same result as when starting all transitions in the correct dominant rotamer (Figure 11c, discard 80). We attribute this to the fact that the alternate rotamer ought to be present to a vanishingly small degree at equilibrium.

Not only is it important to start the transitions with correctly populated orientations of the Val111 side chain, but the switching also has to be slow enough to allow for rearrangement towards the orientation in the other end state at least in some transitions. Note that it is also possible that, with sufficiently large end state ensembles which populate minor rotamers at exactly the right degree, such transitions during switching might not be necessary, but that was not observed here. Unfortunately, it is not obvious how the NEQ switching rate couples to the rate of side chain reorientation, making it difficult to know the required transition length $a$ priori. As discussed in Sec. 5.2.2, although not all non-equilibrium switches have to sample this reorientation, it has to be sampled in some transitions to obtain overlapping work distributions and a reliable free energy estimate. Here, even with $4 \mathrm{~ns} /$ transition, the side chain reoriented only in $8 \%$ of the transitions in the coupling direction (B2A) and $74 \%$ during decoupling of the ligand (A2B), which results in too few transitions sampling the correct rotamer in the coupling direction. Since the conformational change was not sampled sufficiently in $400 \mathrm{~ns}$ total simulation time, we decided that it was better to restrain the side chain.

To support our findings that the large dissipation (SI Figure S9) was caused by a side chain rearrangement upon binding and not by the Boresch-style restraints, we performed calculations applying a lower force constant on the orientational (Boresch-style) restraints (see SI Figure S10). The average dissipated work $W_{d}$ in the forward and reverse directions was similar for the protocols using weaker and stronger restraints (3.7 vs. $3.9 \mathrm{kcal} / \mathrm{mol}$ in the reverse direction and 3.4 vs. $4.1 \mathrm{kcal} / \mathrm{mol}$ in the forward direction). These findings suggests that the Boresch-style restraints were not a major source for the dissipation in this system. 


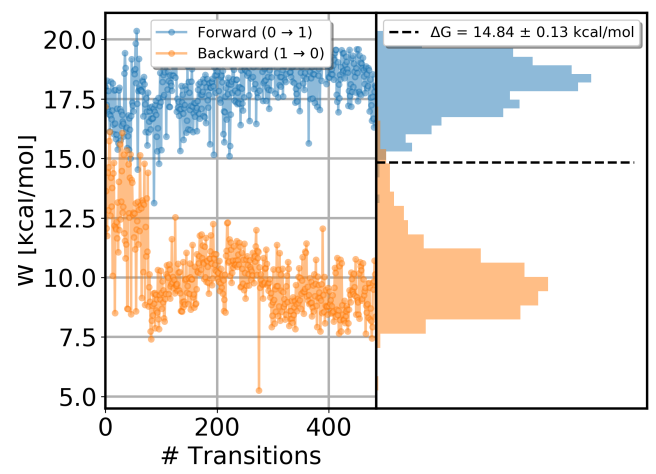

(a)

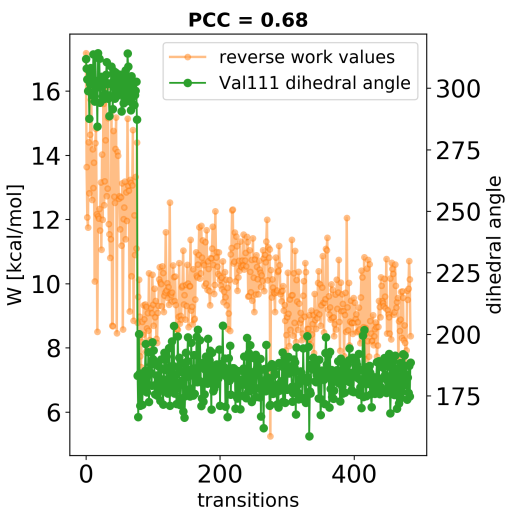

(b)

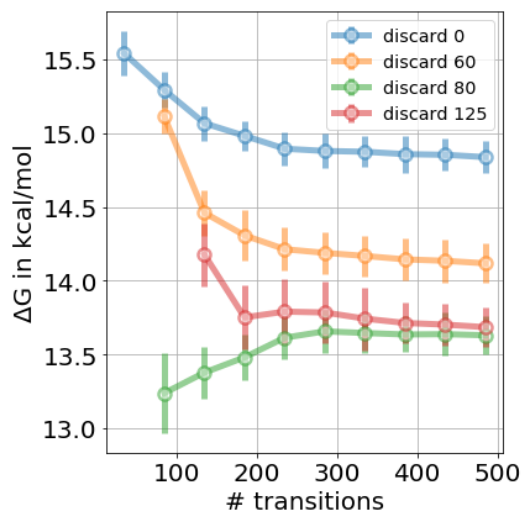

(c)

Figure 11. (a) Work values for 3-iodotoluene binding to T4 lysozyme L99A, in forward (blue) and reverse (orange) direction. Shown are values measured for each attempted transition, as well as the distribution of the work values. In contrast to the general protocol (Figure 10) where $5 \mathrm{~ns}$ of the end state simulation were discarded, here no data were discarded for equilibration. Data from trials in the reverse direction (coupling) shows a sudden change in work values at transition 77. This indicates sampling of a slow motion in the end state. (b) The reverse work values (orange) and the $\xi 1$ dihedral angle of Val111 (green) as a function of transition number. The rotation of the Val111 side chain is correlated with the NEQ work (Pearson correlation coefficient $\mathrm{PCC}=0.68$ ). The sudden change in work values is likely caused by the reorientation of Val111 in the end state simulation. (c) Free energy difference as a function of transition number. The analysis was performed for discarding $0,60,80$ or 125 transitions to study the impact of the Val111 orientation on the final $\Delta G$ estimate. The final free energy estimate is highest when no frames were discarded for equilibration, underestimating the contribution of protein strain relaxation upon unbinding. When 17 transitions start from the wrong V111 rotamer (discard 60, reorientation at transition 77 ), the $\Delta G$ estimate did not converge to the same result as when starting all transitions in the dominant rotamer (discard 80 and 125). This shows the impact of the orientation of the Val111 side chain on the free energy difference.

Equilibrium FEC also suffered from sampling problems due to the rearrangement of the Val111 side chain. These issues were most severe in intermediate $\lambda$ windows where the Val111 side chain showed partial occupancy of two rotamers and a slow rotation between them. In order to investigate this, we looked for sudden changes in $\mathrm{dH} / \mathrm{d} \lambda$ values. Correlating these with the dihedral angle $\xi 1$ of Val111 and comparing results among three independent replicates shows that the side chain was kinetically trapped in various intermediate states (Figure 12). Approaches to overcome these sampling problems include the use of enhanced sampling techniques, like HREX or incorporating BLUES-like side chain moves. ${ }^{57,75}$

\subsubsection{We restrained the Val111 side chain to avoid the sampling of slow DOF.}

In this work, we decided to work around inadequate sampling of this motion by restraining the dihedral angle of the Val111 side chain for both EQ and NEQ approaches. We restrained the side chain to its preferred orientation in the bound and in the decoupled state and switched it during the alchemical path.

With this protocol, the work distributions in the NEQ case overlapped well already using 500 ps/transition (SI Figure S13), indicating that indeed the Val111 side chain sampling led to poor overlap in the unrestrained calculations. However, a lot of NEQ transitions ( 320, SI Figure S12) were necessary in order to converge independent replicates which was probably due to additional slow DOF in the system (perhaps Ile78 rotamer sampling). The approach of switching the dihedral during the alchemical path was challenging using the EQ protocol. The standard deviation across replicates was higher $(\mathrm{std}=0.4 \mathrm{kcal} / \mathrm{mol}$, SI Figure S11) than in the NEQ approach $(\mathrm{std}=0.07 \mathrm{kcal} / \mathrm{mol})$ caused by insufficient sampling of the Val111 switching in some intermediate states.

Since we restrained the dihedral, to calculate binding free energies, we must account for the energetic costs of the dihedral restraints in the thermodynamic cycle. We did this by first turning the restraints on with the ligand interacting 

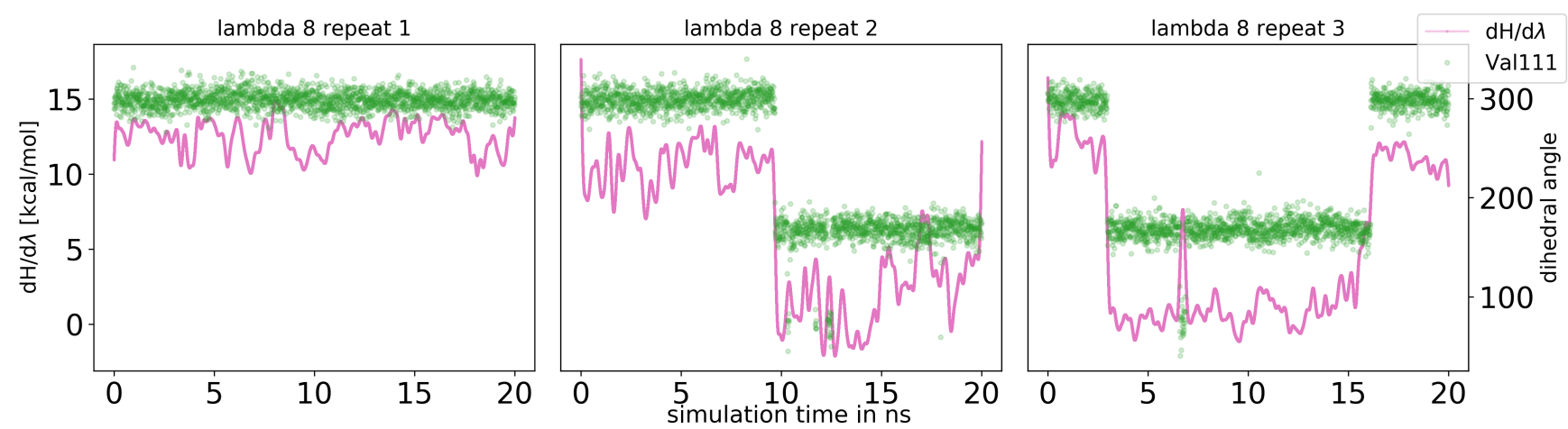

Figure 12. Correlation between $\mathrm{dH} / \mathrm{d} \lambda$ values and the dihedral angle of Val111 for 3-iodotoluene binding to T4 lysozyme L99A. A running average of the $\mathrm{dH} / \mathrm{d} \lambda$ values (pink, averaged across 2000 data points) and the dihedral angle of Val111 (green) is plotted as a function of simulation time. Here we only show $\lambda 8$ for the three independent replicates, additional $\lambda$ windows can be found in the SI. Rotation of the Val111 side chain resulted in sudden changes of $\mathrm{dH} / \mathrm{d} \lambda$ showing the impact of the side chain orientation on the free energy difference. The side chain did not rotate in repeat 1 , while it did in the other two repeats indicating that the correct equilibrium distribution was not sampled adequately in $20 \mathrm{~ns}$ simulation time.

in the binding site, then decoupling the ligand while simultaneously switching the restraints to the orientation in the apo structure. Finally the restraints were then turned off again. The cost for restraining the side chain was very low $(0.1$ $\mathrm{kcal} / \mathrm{mol}$ for restraining and $-0.1 \mathrm{kcal} / \mathrm{mol}$ for unrestraining) since Val111 was restrained to its preferred orientation in both the holo and apo structure.

We also tested a different protocol, where we restrained the dihedral to the orientation in the holo structure both in the bound and unbound state and did not switch it upon decoupling of the ligand. This introduced new sampling problems: Other side chains in the binding site (methionine 102 , valine 103 , isoleucine 78 ) reoriented in response to the forced unfavorable orientation of Val111 in the unbound state (probably to fill the space which would otherwise be empty) making it necessary to restrain those as well. The Pearson correlation coefficient between the dihedral angles and the work values helped detect some of these DOF. This metric was however sometimes subject to noise since many dihedral angles correlated with work values. Correlations were also not always consistent across replicates because different side chain caused problems in different replicates. Restraining the dihedral angles of those side chains as well led to a low standard deviation $(0.2 \mathrm{kcal} / \mathrm{mol})$ in the NEQ approach already with short transitions (100 ps/transition).
With these additional restraints, it was more challenging to account for the restraining costs. More side chains were restrained and the costs for restraining those to an unfavorable rotameric state in the unbound state were high $(\sim 7.5 \mathrm{kcal} / \mathrm{mol}$, see SI Figure S14).

Although we are aware that the use of restraints is not a good general solution to inadequate side chain rotamer sampling, we think that it helps illustrate a potential pitfall of these approaches. Particularly, restraining the side chains gives the same outcome one might get in a shorter simulation, or a simulation where the environment is more sterically constrained (in which the side chain might not switch rotamers at all). In such cases, one might see "good overlap" in the non-equilibrium work distributions but only because the simulations missed an important motion that needs to be sampled.

\subsection{Summary of the T4 lysozyme sys-} tem. In the T4 lysozyme cases examined, convergence of the $\Delta G$ estimate was challenging due to side chain rotamer sampling. Slow side chain rearrangements, like Val111 in the 3iodotoluene case, proved to be very challenging both in EQ and NEQ approaches. The length of NEQ transitions became very important here. Sufficient sampling of faster side chain-rearrangements upon binding, like Ile78 in the toluene system, was achieved by running longer/many NEQ transitions and long equi- 
libration in the equilibrium FEC. In principle this could be true for slower rearrangements too, but in some cases the relevant motions can take nanoseconds to hundreds of nanoseconds for a single transition, making it less desirable to simply run longer simulations. In the NEQ approach, sampling problems were detectable from work value trends and their correlation with structural degrees of freedom in equilibrium snapshots they were derived from. Here, we restrain the dihedral angles of the side chains to work around the issues but our findings suggest that better and more robust methods are necessary to solve the problem.

\subsection{Free energy calculations in the} HSP90 system. In the HSP90 system, in both EQ and NEQ calculations, independent replicates did not converge to the same free energy estimate within a standard deviation of $0.2 \mathrm{kcal} / \mathrm{mol}$ - even with $\sim 1 \mu s$ sampling time per replicate - indicating inadequate sampling (Figure 13). In addition to the high standard deviation among replicates and convergence of the cumulative $\Delta G$ estimate, several additional factors suggest the presence of one or more slow degrees of freedom in the system.

In the NEQ approach, the overlap of the work distributions was very poor, even with $10 \mathrm{~ns} /$ transition (SI Figure S15). Running 10 ns per transition requires considerable computational expense and removes much of the benefit of the NEQ approach. Given the same total simulation time for different NEQ protocols, the lengths of the switching transitions had a huge impact on the estimated free energy change and different protocols only converged to the same result within large uncertainty and after long simulation times. This suggests that the uncertainty of the $\Delta G$ estimates is larger when work distributions lack overlap.

On the other hand, for the EQ approach, the final $\Delta G$ estimate depended on the equilibration time (how much data were discarded to equilibration). Discarding more data (20 ns per $\lambda$ window) made a difference of $\sim 1 \mathrm{kcal} / \mathrm{mol}$ in all three replicates compared to not discarding any data to equilibration (SI Figure S16). This and a low number of uncorrelated samples in some $\lambda$ windows can indicate a slow degree of

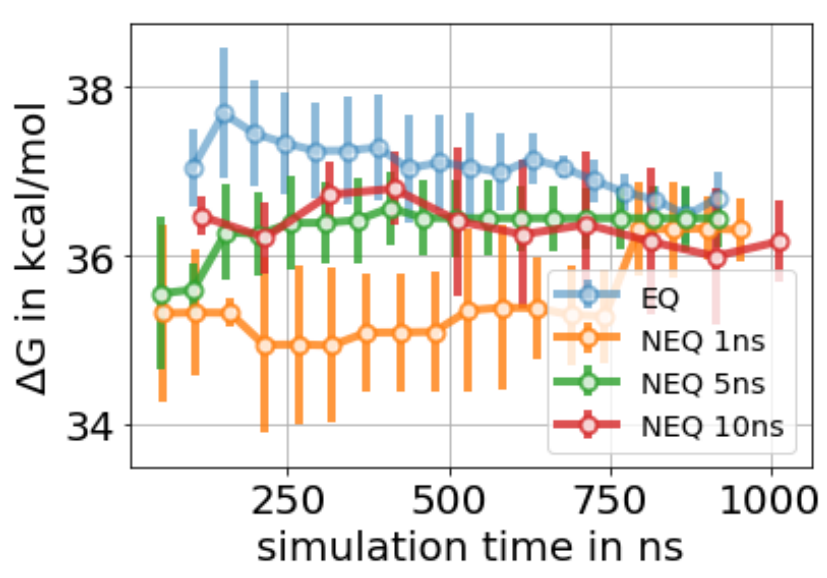

Figure 13. Free energy difference of decoupling the HSP90 ligand in the binding site as a function of total simulation time. For the EQ approach we show the mean free energy difference across three replicates while for the NEQ approach the free energy estimate was obtained by pooling work values from three replicates and estimating one free energy difference using BAR (Sec. 4.5). The uncertainty estimate in both approaches is the standard deviation across three replicates. The three NEQ protocols differ in the length and number of the switching transitions. A high standard deviation indicates inadequate sampling.

freedom (DOF). Similar to the T4 lysozyme system, analyzing sudden changes in $\mathrm{dH} / \mathrm{d} \lambda$ values helped identifying these slow DOF, as shown below.

The overall binding free energy of the HSP90 ligand, which was obtained through the thermodynamic cycle, varied between -11.0 and -11.8 $\mathrm{kcal} / \mathrm{mol}$ among different protocols, as shown in SI Table S6. The binding free energy of the protocols, where three buried water molecules were not present in the starting structure (Sec. 5.5.4), differs from the other protocols by $\sim 2$ $\mathrm{kcal} / \mathrm{mol}(-8.6$ to $-9.9 \mathrm{kcal} / \mathrm{mol})$. These protocols agreed best with the experimental binding free energy of $9.34+/-0.07 \mathrm{kcal} / \mathrm{mol}$, although inadequate sampling was most severe. This is possible since agreement with experimental values is not only a function of adequate sampling, but also of the force field and other factors. Therefore, we focus on whether we have obtained converged values rather than on agreement with experimental values.

5.5.1. Slow water sampling in the solvent exposed binding site of HSP90. The first issue we identified was slow water sampling 
in this solvent exposed binding site. In the unbound state, water molecules completely fill the binding site. Upon binding the ligand displaces the solvent molecules except three buried water molecules between the ligand and the protein (Figure 3).

In equilibrium FEC, inadequate water sampling was mainly observed in the intermediate $\lambda$ windows. As the interactions of the ligand were decoupled, more water molecules entered the space between the ligand and the protein, joining the three buried water molecules (Figure 14). However, since the ligand was still

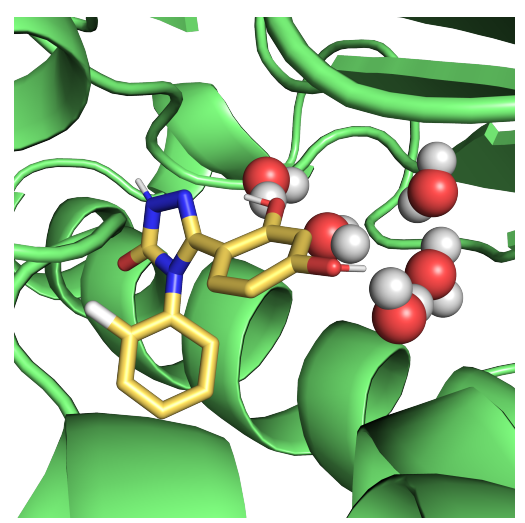

Figure 14. In the HSP90 system, water molecules got trapped in the binding site when the ligand appeared quickly during NEQ transitions as well as in intermediate $\lambda$ windows in the EQ approach. HSP90 is shown in green, ligand in yellow sticks and water molecules are depicted as spheres.

partly interacting and blocking or slowing solvent access to this space, this motion was slow to sample. For multiple $\lambda$ windows, the number of water molecules in the binding site differed among the independent replicates, and $40 \mathrm{~ns}$ sampling time $/ \lambda$ window was not always sufficient to reach equilibrium. Jumps in $\mathrm{dH} / \mathrm{d} \lambda$ curves correlated with water molecules entering the buried space (Figure 15). This suggests that sampling the correct number of waters in the binding site has an impact on the $\Delta G$ estimate. For the fully decoupled ligand, the timescales of the water molecules filling the empty binding site were relatively fast (roughly a few hundred ps).

In the NEQ approach, water molecules got trapped in the same buried space that caused problems in the EQ approach (Figure 14). Particularly, when the ligand appeared in the solvent-filled binding site too quickly, water molecules were not displaced properly and got stuck between the ligand and the protein. This led to poor overlap of the probability distributions of forward and reverse work values and therefore an imprecise (and potentially biased) estimate of the free energy difference. Long transitions were necessary to properly displace the water molecules upon ligand binding. When running each transition for 10 ns, most transitions (63\%) correctly ended up with the three buried water molecules in the binding site, for shorter transitions less did so (5 ns/transitions: $41 \%$ and $1 \mathrm{~ns} /$ transition $7 \%$ ). But such transitions are computationally expensive and remove much of the benefit of the non-equilibrium approach.

\subsubsection{We identified other slow DOF in} this system. A second sampling problem in this system was a binding mode flip that occurred when the ligand was decoupled or only weakly interacting (Figure 17). In this case, orientational restraints only included atoms from two of the three aromatic rings of the ligand (see spheres, Figure 17), hence the third ring was able to flip to a different location.

This only occurred in the weakly interacting states of the ligand, but affected the free energy difference as shown in the correlation between $\mathrm{dH} / \mathrm{d} \lambda$ values and the RMSD of the ligand (Figure 18). The binding mode flip also had an impact in the NEQ approach as judged by a high PCC $(\mathrm{PCC}=0.61)$ between reverse work values and the ligand RMSD (SI Figure S17). To prevent this conformational change in the ligand, one could change the ligand atoms involved in the orientational restraints to also include the ring that flipped in the binding site in order to stabilize the binding mode better in the weaklyto non-interacting alchemical region. Another option would be the use of RMSD restraints. ${ }^{76}$

Finally, minor problems were caused by side chain rearrangement of asparagine 36 (Asn36) upon binding. But since reorientation seemed not to be directly correlated with $\mathrm{dH} / \mathrm{d} \lambda$ values nor to the work values in the NEQ approach, the impact of this DOF on the estimate of the free energy differences is not clear. However, this Asn36 rearrangement does have a 

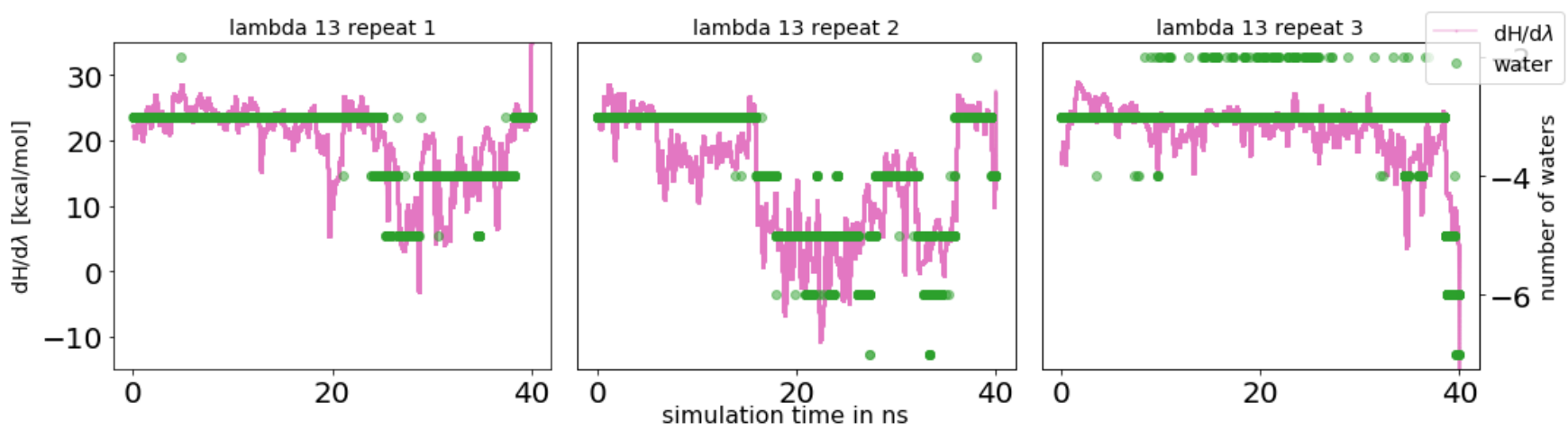

Figure 15. Correlation between $\mathrm{dH} / \mathrm{d} \lambda$ values and the number of water molecules in the binding site fo HSP90. A running average of $\mathrm{dH} / \mathrm{d} \lambda$ values (pink, averaged across 2000 data points) and number of water molecules (green, negative number to better show the correlation) as a function of simulation time per $\lambda$ window. Here we show $\lambda 13$ across all three replicates as an example; additional data is in the SI. The entry of water molecules (more than the three that were already present in the starting structure) was a slow motion and had an impact on the potential energy of the system. Equilibrium was not reached at this particular $\lambda$ window in any of the three repeats.

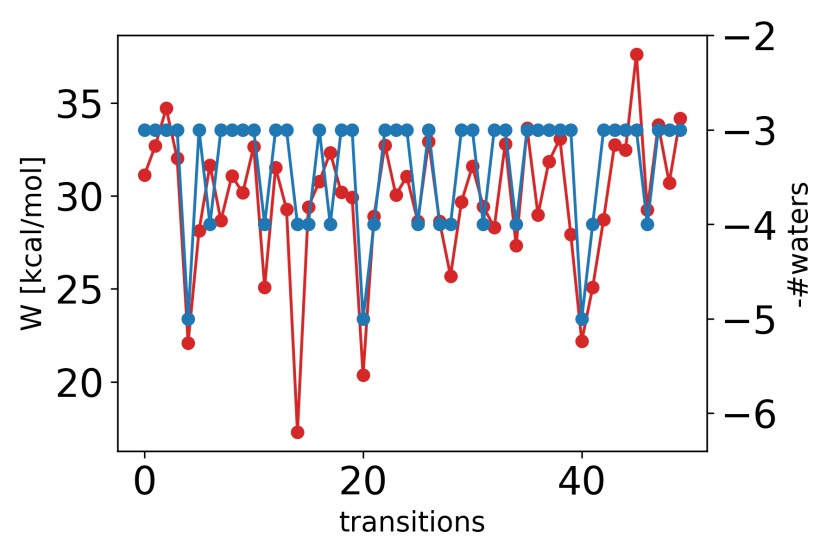

Figure 16. Correlation between NEQ work values (red) and the number of water molecules (blue, with negative numbers to better show the correlation) in the binding site of HSP90 as a function of transition number. Transitions - appearing the ligand in the binding site - were performed for $10 \mathrm{~ns}$ and the last frame of that switching transition was used for counting the number of water molecules. Most of the transitions ended up with three binding site waters, which is correct.

huge impact on the $\Delta G$ estimate in the "bubble approach" as described in Section 5.5.3. It is not clear whether the sampling problem here is overshadowed by more severe problems in water and binding mode sampling, or whether it is directly linked to the bubble approach.

Similar to the T4 lysozyme systems, we checked the impact of the Boresch-style restraints on the dissipation by running calculations with a weaker force constant (1/4 of the original restraint strength). SI Figure S21 shows the comparison of these two protocols.

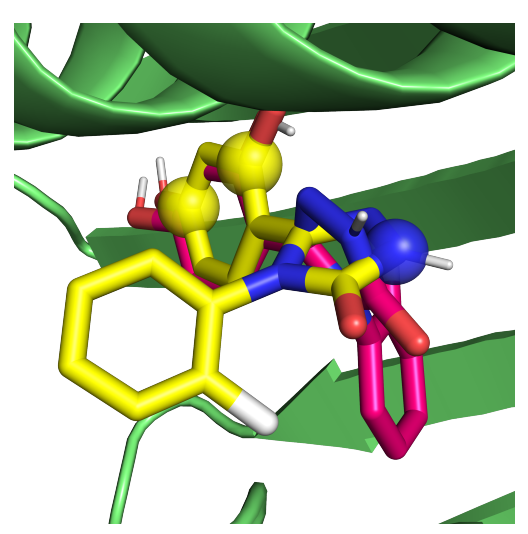

Figure 17. Binding mode flip of the weakly interacting ligand in HSP90. The crystallographic binding mode is shown in yellow, the flipped binding mode in pink and the protein in green. The three ligand atoms that are included in the orientational restraints are represented as spheres. The one aromatic ring that was not included in the restraints flipped into the binding pocket in the weakly and non-interacting alchemical states.

We found that reducing the restraint strength did not have a huge impact on dissipation and the overlap of the work distributions. The average dissipated work $W_{d}$ in the reverse process was $18.7 \mathrm{kcal} / \mathrm{mol}$ for the protocol with a weaker force constant and $15.2 \mathrm{kcal} / \mathrm{mol}$ for the original protocol and in the forward direction 11.5 vs. $13.9 \mathrm{kcal} / \mathrm{mol}$, respectively.

\subsubsection{Addressing inadequate water sam-} pling. The water sampling problem can be separated from the decoupling of the ligand by introducing a bubble-ligand that repels the water and keeps it out of the binding site. This 


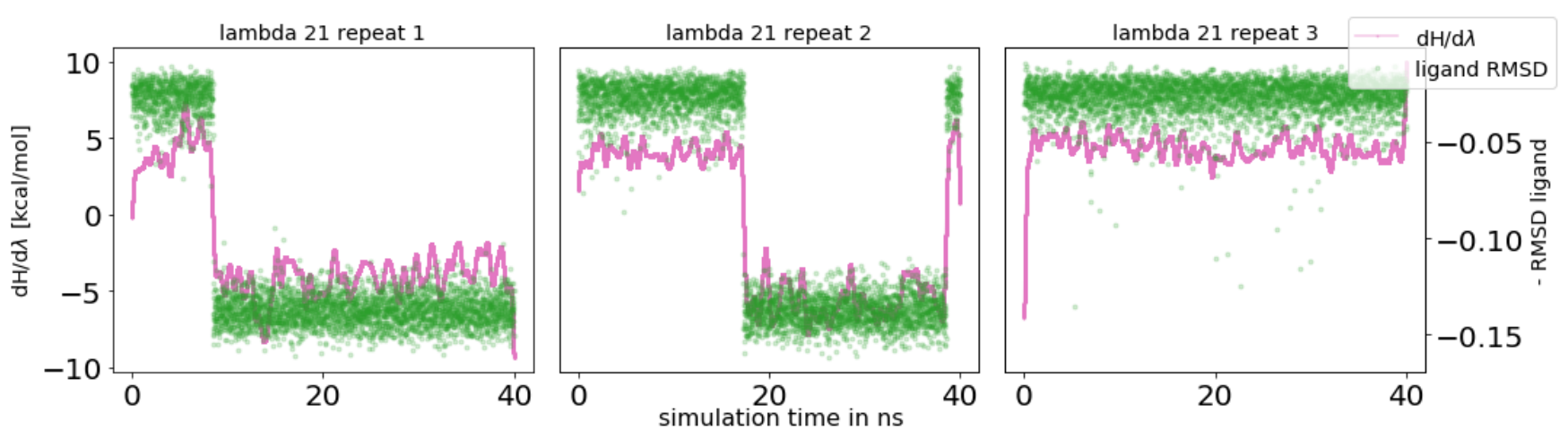

Figure 18. Running average of $\mathrm{dH} / \mathrm{d} \lambda$ values (pink, averaged across 5000 data points) and the RMSD of the HSP90 ligand (green) as a function of the simulation time. We show data for $\lambda 21$ (second last state) for three independent replicates as an example, figures of all $\lambda$ windows can be found in the SI. The correlation between $\mathrm{dH} / \mathrm{d} \lambda$ values and the RMSD of the ligand indicates that the binding mode flip had an impact on the $\Delta G$ estimate, even though these only occurred when the ligand was weakly interacting.

bubble-ligand sits on top of the normal ligand but interacts only with the water molecules (Figure 5). The three buried water molecules were chosen not to interact with this bubbleligand and were restrained with position restraints. This bubble-ligand reduces the dissipation in NEQ and overcomes sampling problems in the EQ approach. Even though the water problem should be accounted for in the NEQ theorem, we found that large dissipation required long transition times or a huge number of switching transitions which became computationally expensive. Our hope was that this approach might isolate some water sampling problems. However, we found that when the ligand was decoupled, the bubble-ligand created a vacuum in the binding site which created its own problems. Side chains of a loop close to the binding site moved in to this vacuum making it necessary to restrain their backbone atoms as well.

Running NEQ with this bubble approach resulted in overlapping work distributions (already with 1 ns/transition, see SI Figure S18) which supports our hypothesis that water sampling was causing the overlap problems. However, in both the EQ and NEQ approaches the standard deviation across independent replicates was high $(0.3-0.5 \mathrm{kcal} / \mathrm{mol}$, SI Figure S19). This was due to slow side chain rearrangement of Asn36 (Section 5.5.2). Rotamer populations of this side chain in the unbound state differed among independent replicates which showed that sampling time was not sufficient to sample the correct Boltzmann weight of the rotamers (see SI). Both NEQ work values and $\mathrm{dH} / \mathrm{d} \lambda$ values in the EQ approach correlated with the orientation of this side chain (SI).

In order to obtain the binding free energy, this bubble approach has to be included in the thermodynamic cycle (Figure 4, right). This makes the approach not only computationally demanding, but also difficult to generalize. The protocol had to be improved iteratively, gearing it towards this specific system. We had to optimize the van-der-Waals parameters of the bubble-ligand to sufficiently repel the water. We also had to restrain the loop that moved into the binding site to fill the vacuum created by the bubble (Sec. 4.). For a different system it would be difficult to predict such structural changes a priori. Additionally, water sampling problems, although less severe, were still present when turning the bubble-ligand off (SI Figure S20). The approach only separated the water sampling challenges from the decoupling of the ligand, but did not solve the problem directly. These factors suggest this approach is not a good general strategy but our results further support our hypothesis that water sampling caused issues in both EQ and NEQ calculations.

The overall binding free energy obtained with this bubble approach is statistically equivalent to the binding free energy obtained with the standard protocol (SI Table S7). The uncer- 
tainty, however, is slightly higher with the bubble approach, partly because four additional legs in the thermodynamic cycle (Figure 4) combine to increase the overall uncertainty.

\subsubsection{The enhanced sampling technique} HREX was not able to overcome inadequate sampling. Enhancing sampling in the EQ approach using HREX did not lead to major improvements with respect to convergence and standard deviation. As shown in Figure 19, the standard deviation is actually higher using HREX $(0.5 \mathrm{kcal} / \mathrm{mol})$ than without enhanced sampling $(0.3 \mathrm{kcal} / \mathrm{mol})$, as we explore below.

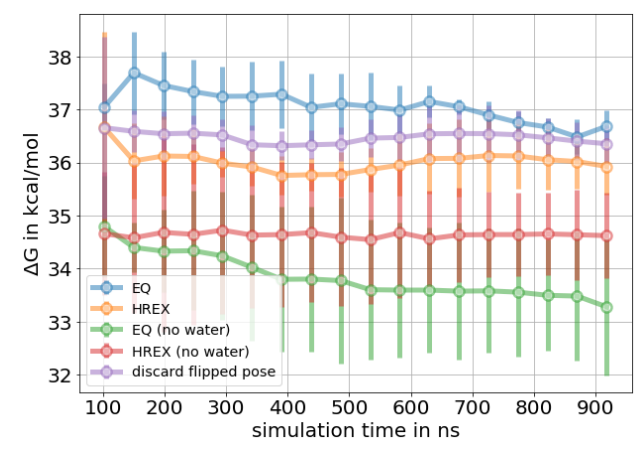

Figure 19. Mean free energy of the ligand binding to HSP90 as a function of total simulation time. We show the standard EQ approach (blue) and the same EQ protocol, but enhancing the sampling with HREX (orange). The purple line shows the free energy estimate of the HREX protocol after discarding all data that included the flipped binding mode. The flipped binding mode had an impact on the $\Delta G$ estimate. A high standard deviation on the HREX protocol indicates that the sampling enhancement here came with its own disadvantages. Green and red lines show simulations where the three buried water molecules were not present in the starting structure. The free energy differences from these calculations differs from the simulations where the buried waters were present (blue, orange, purple) which shows the impact of the binding site waters on the $\Delta G$ estimate.

Both water sampling and the rearrangement of the Asn36 side chain were improved by HREX mixing of replicates. Sampling of the correct binding mode, however, seemed to be more challenging, since unfavorable (flipped) binding modes were passed between different alchemical states through replica exchange swaps (SI). This was possible because some alternate binding modes were favorable enough to be swapped to alchemical states retaining significant interactions and became temporarily trapped, but not so favorable that they ought to be populated substantially at equilibrium. We reran the analysis after discarding all data that included the flipped binding mode. This changed the estimate of the free energy change by $0.4 \mathrm{kcal} / \mathrm{mol}$ and shows the impact of the binding mode flip (Figure 19).

In order to explore whether the NEQ approach benefits from enhanced sampling in the equilibrium end states, we performed NEQ switches using the structures from the EQ HREX run as input. As can be seen in SI Figure S22, using enhanced sampling in the end state did not improve results here. On the contrary, some simulations appearing the ligand crashed. NEQ switches starting from the flipped binding mode, which was sampled more frequently in the HREX protocol (as discussed above), sometimes sampled a binding mode where both phenyl rings switched position (SI Figure S23). This caused those simulation to crash as interaction of the ligand got stronger.

The presence of three buried water molecules in the binding site in the starting structure was crucial for both the EQ and NEQ approach. Indeed, not including these crystallographic waters in the starting structure had a great impact on the computed free energy difference as shown in Figure 19. Only when the ligand was already mostly decoupled, water molecules entered the binding site (SI). Using HREX in the EQ approach did not enhance the sampling sufficiently (Figure 19, red line), and other enhanced sampling methods, like $\mathrm{GC} / \mathrm{MC}$ or $\mathrm{MC} / \mathrm{MD}$, are likely necessary here ${ }^{77,78}$ unless far longer simulations are used, which would raise computational costs far beyond what is currently standard for these calculations. ${ }^{5,6,79}$ Interestingly, inserting the ligand with NEQ transitions "found" these three water molecules in exactly the same positions as in the crystal structure.

Overall, it seems likely that the use of enhanced sampling methods like HREX impacts sampling in a complex way and will affect equilibrium and nonequlibrium free energy calculations differently. While it seems certain that HREX will help dramatically in some cases, in our tests here it does not provide dramatic ben- 
efits in terms of overall sampling quality and accuracy.

\subsection{Summary of sampling problems in} the HSP90 system. In HSP90, water sampling in a solvent exposed binding site caused major problems in alchemical FEC. For the EQ approach, sampling of the correct number of waters in alchemical intermediate states was most challenging while in the NEQ calculations water molecules got trapped in the binding site when quickly coupling the ligand. We attempted to work around the water sampling problem by introducing a bubble-ligand that keeps the water from coming into the binding site. This approach worked to some extent and helped us understand the impact of the water sampling problem better, but is not a good general protocol and more robust methods are required to solve this issue. For both methods, it was crucial to start calculations from protein structures that had three buried binding site water molecules present. Besides the inadequate water sampling, ligand binding mode flips and slow side chain rearrangement caused sampling problems as well. These severe sampling problems highlight the limitation of both approaches in ABFE calculations and reveal clear directions for further investigation.

\section{Discussion and Conclusions}

In this work we highlight challenges encountered applying equilibrium and non-equilibrium binding free energy calculations. We provide insight into how to identify sampling problems and explore similarities and differences between these two methods in handling these issues.

Alchemical calculations frequently pose sampling problems for both the EQ and NEQ approach. We observed slow side chain rearrangements (T4 lysozyme), water sampling in a solvent exposed binding site (HSP90) and flips in ligand binding mode (HSP90). These problems are likely to show up in other protein-ligand systems and may also effect other techniques for binding free energy calculations.

\subsection{Challenges encountered here in} ABFE will probably effect RBFE as well. Some of these challenges will probably affect relative binding free energy calculations
(RBFE) as well. For instance, slow side chain rearrangements will pose challenges whenever different ligands induce slow side chain rotations, as in the case of toluene vs. 3iodotoluene, here, which are not that dissimilar from $\mathrm{R}$-groups that might be modified in RFBE calculations on a larger, drug-like ligand. Water sampling can also cause severe issues in relative binding free energy calculations when a buried water molecule is present with one ligand and displaced by another one. ${ }^{77,80}$ If ligands have a similar shape or displace the same water molecules, RBFE will probably converge faster than the absolute protocol. Binding mode flips, on the other hand, are less likely to occur with RBFE since those happen mostly in the alchemical states where the ligand is only weakly interacting in the binding site. Still, the same issue can affect $\mathrm{R}$-groups of drug-like ligands.

\subsection{Strategies to assess sampling prob-} lems in alchemical FEC. Sampling problems in these systems helped us to establish some general strategies on how to diagnose and test for problems in alchemical FEC. First, we found that in both EQ and NEQ, running independent replicates is important to identify inadequate sampling and to obtain uncertainty estimates. The analytical error - as well as bootstrapping in NEQ - underestimated the true error.

In the EQ approach, the impact of the equilibration time on the $\Delta G$ estimate and autocorrelation analysis can give a hint to the presence of slow DOF in the system (Sec. 5.5).

To identify slow motions in the system that can lead to inadequate sampling, we found it helpful to examine $\mathrm{dH} / \mathrm{d} \lambda$ values and their relationship to other degrees of freedom. We do this by identifying sudden changes in $\mathrm{dH} / \mathrm{d} \lambda$ values as simulations progress. These jumps then tell us when exactly to look for sudden/slow motions by analyzing frames near those jumps. Once a slow motion is identified, the correlation between the motion and the $\mathrm{dH} / \mathrm{d} \lambda$ values across all alchemical states can be easily investigated. This method was successfully applied for the three ligands in this paper and helped identify sampling problems. The approach of detecting sampling issues affecting $\mathrm{dH} / \mathrm{d} \lambda$ val- 
ues could be automated in the future, including perhaps with machine learning, e.g. using functional mode analysis.

The overlap of forward and reverse work distributions provides a helpful further diagnostic for the NEQ approach. Having sufficient overlap of the distributions is necessary, but not sufficient for obtaining unbiased free energy estimates; as we found in Section 5.3.2, in some cases, inadequate sampling leads to a false appearance of good overlap. Slow degrees of freedom also often lead to poor overlap, which results in a high analytical error estimate. In addition, bimodal work distributions and sudden changes in work values can be a warning sign that sampling might be complex and slow. Calculating the cross-correlation between side chain dihedral angles in the protein and the non-equilibrium work values can help to diagnose problems. Instead of dihedral angles one could also choose other metrics for protein motion, such as side chain RMSD values. It is important to mention however, that analysis of correlations is prone to false positives - both for correlations between DOF and $\mathrm{dH} / \mathrm{d} \lambda$ curves in the EQ approach and DOF with work values in the NEQ approach. It should therefore be applied with caution and the use of replicates for cross-correlation.

In a solvent exposed binding site, counting the number of water molecules in the binding pocket can help detect changes in water placement and occupancy on binding. We also found that it is crucial to include crystallographic water molecules, especially when coming from a high resolution structure with clear density showing ordered waters in the binding site. Here, this was important because the water molecules were buried in the binding site and the timescale for entering the buried space was very slow.

Although not exploited in this paper, it can be a good strategy to run simulations starting in apo and holo structures if both are available. Since both calculations should converge to the same answer this can help to determine when sampling is sufficient. ${ }^{47,81}$

We attempted to address these sampling problems and discovered and found ways to work around some of them. We restrained side chains and introduced a bubble approach for water sampling. However, better solutions are needed to make alchemical FEC more general and robust. In the long-term, greater use of enhanced sampling techniques is likely needed, such as perhaps BLUES-like moves ${ }^{57,75}$ to accelerate side chain sampling.

The sampling challenges in the systems we investigated made it difficult to directly compare the efficiency of the EQ and the NEQ approaches as we had originally hoped (and as some prior studies have attempted to do ${ }^{42}$ ). For the hydration free energy of the three ligands however, calculations converged sufficiently and we were able to compare the efficiency of the EQ and NEQ approaches. Both methods performed very similar on these systems, but the NEQ approach was able to reduce the standard deviation and the bias faster than the EQ approach, suggesting that it is more efficient here, at least. However, differences were very small, and thus a larger data set is needed to investigate this more.

\subsection{Similarities and differences of the EQ and NEQ approaches in handling slow DOF. For the decoupling of the ligands in the binding site, we concentrated on providing some understanding as to why each method behaves the way it does.}

First, we compared how the two methods handle side chain rearrangements upon binding. A benefit of the NEQ approach is that the equilibrium orientation of the side chain has to be sampled only in the physical end states and not in alchemical intermediates. Crystal structures can give insight here and help assess sufficient sampling.

A weakness, however, is that when end states differ and the conformational change is not sampled during NEQ switching, dissipation can be large and a slow switching rate or numerous transitions may be required to obtain a converged free energy difference. In the case of Val111 sampling in 3-iodotoluene, we saw that this can be very limiting. Possibly separation of states approaches can help with this issue, ${ }^{82}$ e.g. having the side chain restrained during the calculations and accounting for it later. This how- 
ever, creates its own problems in some cases.

An advantage for EQ, on the other hand, is that every state is sampled for a long time, allowing for rearrangements. Every state has to be sampled at equilibrium. When there are slow side chain rotations upon binding it can be difficult to reach equilibrium as well as to detect when equilibrium has been reached. Experimental structures also do not indicate the preferred side chain orientation in the unphysical alchemical region.

Water sampling in a solvent exposed binding site caused problems for both methods. For the NEQ approach, very long transitions times (5 ns - 10 ns per transitions) were required to sample changes in water placement which removed much of the benefit of the non-equilibrium approach.

In our experience, a benefit of the NEQ approach is that it can be easier to do sanity checks. Only two long simulations are performed in the physical states which is easier than having to check all trajectories along the alchemical path in EQ. The long end state simulations also provide a point of reference to check that the transition simulations are capturing the right motions. Specifically, an A $\rightarrow$ B transition needs to (at least some fraction of the time) end up at snapshots that are representative of the same B state seen in the long equilibrium B simulation. This could be done in a systematic and automated way by ensemble comparison techniques.

In the NEQ approach, systematic errors can be introduced by starting NEQ transitions from local minima structures drawn from an incorrect or biased distribution (i.e. if the starting structures overpopulate some conformations and underpopulate others). In these cases, end state simulations can benefit from enhanced sampling techniques. In this work enhanced sampling methods were not investigated in detail in the NEQ approach, since we based this study on work of Gapsys et al. which has been successful without this. ${ }^{79}$ In hindsight, given our results (e.g. Figure 9a), it is worth exploring if faster convergence can be achieved with such enhancements. We tested enhanced end state sampling in the HSP90 system, but start- ing transitions from structures of the EQ HREX calculations did not improve results in this case.

We learned in this study that there is no "free lunch" switching from one alchemical approach to another. If a transition is challenging, the convergence issues will remain for both EQ and NEQ approaches. An advantage of using multiple protocols might be that sampling problems can manifest differently. This may be helpful in identifying where the main sampling issues are and therefore in devising ways to alleviate them.

\section{Disclosures}

DLM is a member of the Scientific Advisory Board of OpenEye Scientific Software and an Open Science Fellow with Silicon Therapeutics.

\section{Supporting Information Avail- able}

We provide details on the free energy calculations in Figures S1-S16 and Tables S1-S5. GROMACS topology and coordinate files and parameter files (MDP files) are provided as a compressed archive file along with example scripts used in the analysis. We also provide additional data for completeness whenever we showed analysis only for a specific lambda window/replicate in the main body of this paper. More detailed explanations on the SI content can be found in README.md files.

Acknowledgement DLM appreciates financial support from the National Institutes of Health (R01GM108889 and R01GM124270) and computing support from the UCI GreenPlanet cluster, supported in part by NSF Grant CHE-0840513. We also appreciate NVIDIA Corporation for donation of some of the GPUs used in this work. The content is solely the responsibility of the authors and does not necessarily represent the official views of the National Institutes of Health. VG and BLdG were supported by BioExcel CoE (www.bioexcel.eu), a project funded by the European Union, contract H2020-INFRAEDI-022018-823830. HMB appreciates the Penny J. 
Gilmer Grant for Women Graduate Students and Post-docs from OpenEye Scientific Software that enabled her to present this work at their annual user meeting. HMB thanks Victoria T. Lim for providing scripts to analyze binding site waters and for reviewing the manuscript and Léa El Khoury for her help preparing input files.

\section{References}

(1) Enamine REAL database: https://enamine.net/library-synthesis/realcompounds/real-database. Accessed 18. Sep. 2020 .

(2) Gorgulla, C.; Boeszoermenyi, A.; Wang, Z.F.; Fischer, P. D.; Coote, P. W.; Padmanabha Das, K. M.; Malets, Y. S.; Radchenko, D. S.; Moroz, Y. S.; Scott, D. A. et al. An open-source drug discovery platform enables ultra-large virtual screens. Nature 2020, 580, 663-668.

(3) Parks, C. D.; Gaieb, Z.; Chiu, M.; Yang, H.; Shao, C.; Walters, W. P.; Jansen, J. M.; McGaughey, G.; Lewis, R. A.; Bembenek, S. D. et al. D3R grand challenge 4: blind prediction of protein-ligand poses, affinity rankings, and relative binding free energies. Journal of Computer-Aided Molecular Design 2020, 34, 99-119.

(4) Sherborne, B.; Shanmugasundaram, V.; Cheng, A. C.; Christ, C. D.; DesJarlais, R. L.; Duca, J. S.; Lewis, R. A.; Loughney, D. A.; Manas, E. S.; McGaughey, G. B. et al. Collaborating to improve the use of free-energy and other quantitative methods in drug discovery. Journal of Computer-Aided Molecular Design 2016, 30, 1139-1141.

(5) Cournia, Z.; Allen, B. K.; Beuming, T.; Pearlman, D. A.; Radak, B. K.; Sherman, W. Rigorous Free Energy Simulations in Virtual Screening. Journal of Chemical Information and Modeling 2020, 60, 4153-4169.

(6) Schindler, C. E. M.; Baumann, H.; Blum, A.; Böse, D.; Buchstaller, H.-P.; Burgdorf, L.; Cappel, D.; Chekler, E.; Czodrowski, P.; Dorsch, D. et al. Large-Scale Assessment of Binding Free Energy Calculations in Active Drug Discovery Projects. Journal of Chemical Information and Modeling 2020, 60, 5457-5474.

(7) Mobley, D. L.; Klimovich, P. V. Perspective: Alchemical free energy calculations for drug discovery. The Journal of Chemical Physics 2012, 137, 230901.
(8) Aldeghi, M.; Heifetz, A.; Bodkin, M. J.; Knapp, S.; Biggin, P. C. Predictions of Ligand Selectivity from Absolute Binding Free Energy Calculations. Journal of the American Chemical Society 2017, 139, 946-957.

(9) Ishima, R.; Freedberg, D. I.; Wang, Y.-X.; Louis, J. M.; Torchia, D. A. Flap opening and dimer-interface flexibility in the free and inhibitorbound HIV protease, and their implications for function. Structure 1999, 7, 1047-S12.

(10) D'Abramo, M.; Besker, N.; Chillemi, G.; Grottesi, A. Modeling conformational transitions in kinases by molecular dynamics simulations: achievements, difficulties, and open challenges. Frontiers in Genetics 2014, 5.

(11) Mobley, D. L.; Gilson, M. K. Predicting Binding Free Energies: Frontiers and Benchmarks. Annual Review of Biophysics 2017, 46, 531-558.

(12) Williams-Noonan, B. J.; Yuriev, E.; Chalmers, D. K. Free Energy Methods in Drug Design: Prospects of "Alchemical Perturbation" in Medicinal Chemistry: Miniperspective. Journal of Medicinal Chemistry 2018, 61, 638-649.

(13) Shirts, M. R.; Mobley, D. L.; Chodera, J. D. Annual Reports in Computational Chemistry; Elsevier, 2007; Vol. 3; pp 41-59.

(14) Mey, A. S. J. S.; Allen, B.; Macdonald, H. E. B.; Chodera, J. D.; Kuhn, M.; Michel, J.; Mobley, D. L.; Naden, L. N.; Prasad, S.; Rizzi, A. et al. Best Practices for Alchemical Free Energy Calculations. arXiv:2008.03067 [q-bio, stat] 2020, arXiv: 2008.03067.

(15) Armacost, K. A.; Riniker, S.; Cournia, Z. Exploring Novel Directions in Free Energy Calculations. Journal of Chemical Information and Modeling 2020, 60, 5283-5286.

(16) Lee, T.-S.; Allen, B. K.; Giese, T. J.; Guo, Z.; Li, P.; Lin, C.; McGee, T. D.; Pearlman, D. A.; Radak, B. K.; Tao, Y. et al. Alchemical Binding Free Energy Calculations in AMBER20: Advances and Best Practices for Drug Discovery. Journal of Chemical Information and Modeling 2020, 60, 5595-5623.

(17) Perthold, J. W.; Petrov, D.; Oostenbrink, C. Toward Automated Free Energy Calculation with Accelerated Enveloping Distribution Sampling (AEDS). Journal of Chemical Information and Modeling 2020, 60, 5395-5406.

(18) Crooks, G. E. Nonequilibrium Measurements of Free Energy Differences for Microscopically Reversible Markovian Systems. Journal of Statistical Physics 1998, 90, 1481-1487. 
(19) Jarzynski, C. Equilibrium free-energy differences from nonequilibrium measurements: A masterequation approach. Physical Review E 1997, 56, 5018-5035.

(20) Crooks, G. E. Entropy production fluctuation theorem and the nonequilibrium work relation for free energy differences. Physical Review E 1999, 60, 2721-2726.

(21) Macchiagodena, M.; Pagliai, M.; Karrenbrock, M.; Guarnieri, G.; Iannone, F.; Procacci, P. Virtual Double-System Single-Box: A Nonequilibrium Alchemical Technique for Absolute Binding Free Energy Calculations: Application to Ligands of the SARS-CoV-2 Main Protease. Journal of Chemical Theory and Computation 2020, 16, 7160-7172.

(22) Khalak, Y.; Tresadern, G.; de Groot, B. L.; Gapsys, V. Non-equilibrium approach for binding free energies in cyclodextrins in SAMPL7: force fields and software. Journal of Computer-Aided Molecular Design 2021, 35, 49-61.

(23) Knight, J. L.; Brooks, C. L. Lambda-Dynamics free energy simulation methods. Journal of Computational Chemistry 2009, 30, 1692-1700.

(24) Gilson, M.; Given, J.; Bush, B.; McCammon, J. The statistical-thermodynamic basis for computation of binding affinities: a critical review. Biophysical Journal 1997, 72, 1047-1069.

(25) Steinbrecher, T.; Labahn, A. Towards Accurate Free Energy Calculations in Ligand ProteinBinding Studies. Current Medicinal Chemistry 2010, 17, 767-785.

(26) Ytreberg, F. M.; Swendsen, R. H.; Zuckerman, D. M. Comparison of free energy methods for molecular systems. The Journal of Chemical Physics 2006, 125, 184114.

(27) Bennett, C. H. Efficient estimation of free energy differences from Monte Carlo data. Journal of Computational Physics 1976, 22, 245-268.

(28) Shirts, M. R.; Chodera, J. D. Statistically optimal analysis of samples from multiple equilibrium states. The Journal of Chemical Physics 2008, 129, 124105.

(29) Kirkwood, J. G. Statistical Mechanics of Fluid Mixtures. The Journal of Chemical Physics 1935, 3, 300-313.

(30) Shirts, M. R.; Pande, V. S. Comparison of efficiency and bias of free energies computed by exponential averaging, the Bennett acceptance ratio, and thermodynamic integration. The Journal of Chemical Physics 2005, 122, 144107.
(31) Woods, C. J.; Essex, J. W.; King, M. A. The Development of Replica-Exchange-Based FreeEnergy Methods. The Journal of Physical Chemistry $B$ 2003, 10\%, 13703-13710.

(32) Sugita, Y.; Kitao, A.; Okamoto, Y. Multidimensional replica-exchange method for free-energy calculations. The Journal of Chemical Physics 2000, 113, 6042-6051.

(33) Woods, C. J.; Essex, J. W.; King, M. A. Enhanced Configurational Sampling in Binding Free-Energy Calculations. The Journal of Physical Chemistry B 2003, 10\%, 13711-13718.

(34) Pal, R. K.; Gallicchio, E. Perturbation potentials to overcome order/disorder transitions in alchemical binding free energy calculations. The Journal of Chemical Physics 2019, 151, 124116.

(35) Sun, Z. X.; Wang, X. H.; Zhang, J. Z. H. BARbased optimum adaptive sampling regime for variance minimization in alchemical transformation. Physical Chemistry Chemical Physics 2017, 19, 15005-15020.

(36) Procacci, P. Solvation free energies via alchemical simulations: let's get honest about sampling, once more. Physical Chemistry Chemical Physics 2019, 21, 13826-13834.

(37) Jarzynski, C. Nonequilibrium Equality for Free Energy Differences. Physical Review Letters 1997, 78, 2690-2693.

(38) Aldeghi, M.; de Groot, B. L.; Gapsys, V. In Computational Methods in Protein Evolution; Sikosek, T., Ed.; Springer New York: New York, NY, 2019; Vol. 1851; pp 19-47, Series Title: Methods in Molecular Biology.

(39) Jarzynski, C. Rare events and the convergence of exponentially averaged work values. Physical Review E 2006, 73, 046105.

(40) Wu, D.; Kofke, D. A. Phase-space overlap measures. I. Fail-safe bias detection in free energies calculated by molecular simulation. The Journal of Chemical Physics 2005, 123, 054103.

(41) Crooks, G. E. Path-ensemble averages in systems driven far from equilibrium. Physical Review $E$ 2000, 61, 2361-2366.

(42) Rizzi, A.; Jensen, T.; Slochower, D. R.; Aldeghi, M.; Gapsys, V.; Ntekoumes, D.; Bosisio, S.; Papadourakis, M.; Henriksen, N. M.; de Groot, B. L. et al. The SAMPL6 SAMPLing challenge: assessing the reliability and efficiency of binding free energy calculations. Journal of Computer-Aided Molecular Design 2020, 34, 601633. 
(43) Dellago, C.; Hummer, G. Computing Equilibrium Free Energies Using Non-Equilibrium Molecular Dynamics. Entropy 2013, 16, 41-61.

(44) Eriksson, A.; Baase, W.; Zhang, X.; Heinz, D.; Blaber, M.; Baldwin, E.; Matthews, B. Response of a protein structure to cavity-creating mutations and its relation to the hydrophobic effect. Science 1992, 255, 178.

(45) Hermans, J.; Wang, L. Inclusion of Loss of Translational and Rotational Freedom in Theoretical Estimates of Free Energies of Binding. Application to a Complex of Benzene and Mutant T4 Lysozyme. Journal of the American Chemical Society 1997, 119, 2707-2714.

(46) Deng, Y.; Roux, B. Calculation of Standard Binding Free Energies: Aromatic Molecules in the T4 Lysozyme L99A Mutant. Journal of Chemical Theory and Computation 2006, 2, 1255-1273.

(47) Mobley, D. L.; Graves, A. P.; Chodera, J. D.; McReynolds, A. C.; Shoichet, B. K.; Dill, K. A. Predicting Absolute Ligand Binding Free Energies to a Simple Model Site. Journal of Molecular Biology 2007, 371, 1118-1134.

(48) Mobley, D. L.; Slochower, D. Mobleylab/Benchmarksets: Version 1.2. 2017; https://zenodo.org/record/839047.

(49) Jiang, W.; Thirman, J.; Jo, S.; Roux, B. Reduced Free Energy Perturbation/Hamiltonian Replica Exchange Molecular Dynamics Method with Unbiased Alchemical Thermodynamic Axis. The Journal of Physical Chemistry B 2018, 122, 94359442 .

(50) Amaral, M.; Kokh, D. B.; Bomke, J.; Wegener, A.; Buchstaller, H. P.; Eggenweiler, H. M.; Matias, P.; Sirrenberg, C.; Wade, R. C.; Frech, M. Protein conformational flexibility modulates kinetics and thermodynamics of drug binding. Nature Communications 2017, 8, 2276.

(51) Huth, J. R.; Park, C.; Petros, A. M.; Kunzer, A. R.; Wendt, M. D.; Wang, X.; Lynch, C. L.; Mack, J. C.; Swift, K. M.; Judge, R. A. et al. Discovery and Design of Novel HSP90 Inhibitors Using Multiple Fragment-based Design Strategies. Chemical Biology \& Drug Design 2007, 70, 1-12.

(52) Boresch, S.; Tettinger, F.; Leitgeb, M.; Karplus, M. Absolute Binding Free Energies: A Quantitative Approach for Their Calculation. The Journal of Physical Chemistry B 2003, 10\%, 9535-9551.

(53) Eastman P $\quad$ (2019) PDBFixer. https://github.com/pandegroup/pdbfixer.
(54) Dolinsky, T. J.; Nielsen, J. E.; McCammon, J. A.; Baker, N. A. PDB2PQR: an automated pipeline for the setup of Poisson-Boltzmann electrostatics calculations. Nucleic Acids Research 2004, 32, W665-W667.

(55) Dolinsky, T. J.; Czodrowski, P.; Li, H.; Nielsen, J. E.; Jensen, J. H.; Klebe, G.; Baker, N. A. PDB2PQR: expanding and upgrading automated preparation of biomolecular structures for molecular simulations. Nucleic Acids Research 2007, 35, W522-W525.

(56) OpenEye Toolkits 2020.1.0 OpenEye Scientific Software, Santa Fe, NM. http://www.eyesopen.com.

(57) Gill, S. C.; Lim, N. M.; Grinaway, P. B.; Rustenburg, A. S.; Fass, J.; Ross, G. A.; Chodera, J. D.; Mobley, D. L. Binding Modes of Ligands Using Enhanced Sampling (BLUES): Rapid Decorrelation of Ligand Binding Modes via Nonequilibrium Candidate Monte Carlo. The Journal of Physical Chemistry B 2018, 122, 5579-5598.

(58) Rizzi, A.; Chodera, J.; Naden, L.; Beauchamp, K.; Grinaway, P.; Rustenburg, B.; Albanese, S.; Saladi, S. Choderalab/Yank: 0.23.0 Multi-Analysis. 2018; https://zenodo.org/record/1304282.

(59) Maier, J. A.; Martinez, C.; Kasavajhala, K.; Wickstrom, L.; Hauser, K. E.; Simmerling, C. ff14SB: Improving the Accuracy of Protein Side Chain and Backbone Parameters from ff99SB. Journal of Chemical Theory and Computation 2015, 11, 3696-3713.

(60) Wang, J.; Wolf, R. M.; Caldwell, J. W.; Kollman, P. A.; Case, D. A. Development and testing of a general amber force field. Journal of Computational Chemistry 2004, 25, 1157-1174.

(61) Jakalian, A.; Jack, D. B.; Bayly, C. I. Fast, efficient generation of high-quality atomic charges. AM1BCC model: II. Parameterization and validation. Journal of Computational Chemistry 2002, 23, 1623-1641.

(62) Jorgensen, W. L.; Chandrasekhar, J.; Madura, J. D.; Impey, R. W.; Klein, M. L. Comparison of simple potential functions for simulating liquid water. The Journal of Chemical Physics 1983, 79, 926-935.

(63) Mobley, D. L.; Chodera, J. D.; Dill, K. A. On the use of orientational restraints and symmetry corrections in alchemical free energy calculations. The Journal of Chemical Physics 2006, 125, 084902. 
(64) Berendsen, H.; van der Spoel, D.; van Drunen, R. GROMACS: A message-passing parallel molecular dynamics implementation. Computer Physics Communications 1995, 91, 43-56.

(65) Abraham, M. J.; Murtola, T.; Schulz, R.; Páll, S.; Smith, J. C.; Hess, B.; Lindahl, E. GROMACS: High performance molecular simulations through multi-level parallelism from laptops to supercomputers. SoftwareX 2015, 1-2, 19-25.

(66) Beutler, T. C.; Mark, A. E.; van Schaik, R. C.; Gerber, P. R.; van Gunsteren, W. F. Avoiding singularities and numerical instabilities in free energy calculations based on molecular simulations. Chemical Physics Letters 1994, 222, 529-539.

(67) Lee, T.-S.; Lin, Z.; Allen, B. K.; Lin, C.; Radak, B. K.; Tao, Y.; Tsai, H.-C.; Sherman, W.; York, D. M. Improved Alchemical Free Energy Calculations with Optimized Smoothstep Softcore Potentials. Journal of Chemical Theory and Computation 2020, 16, 5512-5525.

(68) Shirts, M. R.; Bair, E.; Hooker, G.; Pande, V. S. Equilibrium Free Energies from Nonequilibrium Measurements Using Maximum-Likelihood Methods. Physical Review Letters 2003, 91, 140601.

(69) Klimovich, P. V.; Shirts, M. R.; Mobley, D. L. Guidelines for the analysis of free energy calculations. Journal of Computer-Aided Molecular Design 2015, 29, 397-411.

(70) Dotson, D.; Beckstein, O.; Wille, D.; Kenney, I.; shuail,; trje3733,; Lee, H.; Lim, V.; Allen, B.; Barhaghi, M. S. alchemistry/alchemlyb: 0.3.1. 2020; https://doi.org/10.5281/zenodo. 3610564 .

(71) Gapsys, V.; Michielssens, S.; Seeliger, D.; de Groot, B. L. pmx: Automated protein structure and topology generation for alchemical perturbations. Journal of Computational Chemistry 2015, 36, 348-354.

(72) Seeliger, D.; de Groot, B. L. Protein Thermostability Calculations Using Alchemical Free Energy Simulations. Biophysical Journal 2010, 98, 23092316 .

(73) Shi, Y.; Laury, M. L.; Wang, Z.; Ponder, J. W. AMOEBA binding free energies for the SAMPL7 TrimerTrip host-guest challenge. Journal of Computer-Aided Molecular Design 2021, 35, 79-93.

(74) Jelly beans comic, https://xkcd.com/882/.
(75) Burley, K. H.; Gill, S. C.; Lim, N. M.; Mobley, D. L. Enhancing Side Chain Rotamer Sampling Using Nonequilibrium Candidate Monte Carlo. Journal of Chemical Theory and Computation 2019, 15, 1848-1862.

(76) Woo, H.-J.; Roux, B. Calculation of absolute protein-ligand binding free energy from computer simulations. Proceedings of the National Academy of Sciences 2005, 102, 6825-6830.

(77) Ross, G. A.; Russell, E.; Deng, Y.; Lu, C.; Harder, E. D.; Abel, R.; Wang, L. Enhancing Water Sampling in Free Energy Calculations with Grand Canonical Monte Carlo. Journal of Chemical Theory and Computation 2020, 16, 6061-6076.

(78) Ben-Shalom, I. Y.; Lin, C.; Kurtzman, T.; Walker, R. C.; Gilson, M. K. Simulating Water Exchange to Buried Binding Sites. Journal of Chemical Theory and Computation 2019, 15, 2684-2691.

(79) Gapsys, V.; Pérez-Benito, L.; Aldeghi, M.; Seeliger, D.; van Vlijmen, H.; Tresadern, G.; de Groot, B. L. Large scale relative protein ligand binding affinities using non-equilibrium alchemy. Chemical Science 2020, 11, 1140-1152.

(80) Wahl, J.; Smieško, M. Assessing the Predictive Power of Relative Binding Free Energy Calculations for Test Cases Involving Displacement of Binding Site Water Molecules. Journal of Chemical Information and Modeling 2019, 59, 754-765.

(81) Lim, N. M.; Wang, L.; Abel, R.; Mobley, D. L. Sensitivity in Binding Free Energies Due to Protein Reorganization. Journal of Chemical Theory and Computation 2016, 12, 4620-4631.

(82) Mobley, D. L.; Chodera, J. D.; Dill, K. A. Confineand-Release Method: Obtaining Correct Binding Free Energies in the Presence of Protein Conformational Change. Journal of Chemical Theory and Computation 2007, 3, 1231-1235. 


\section{Hydration free energy calculations}
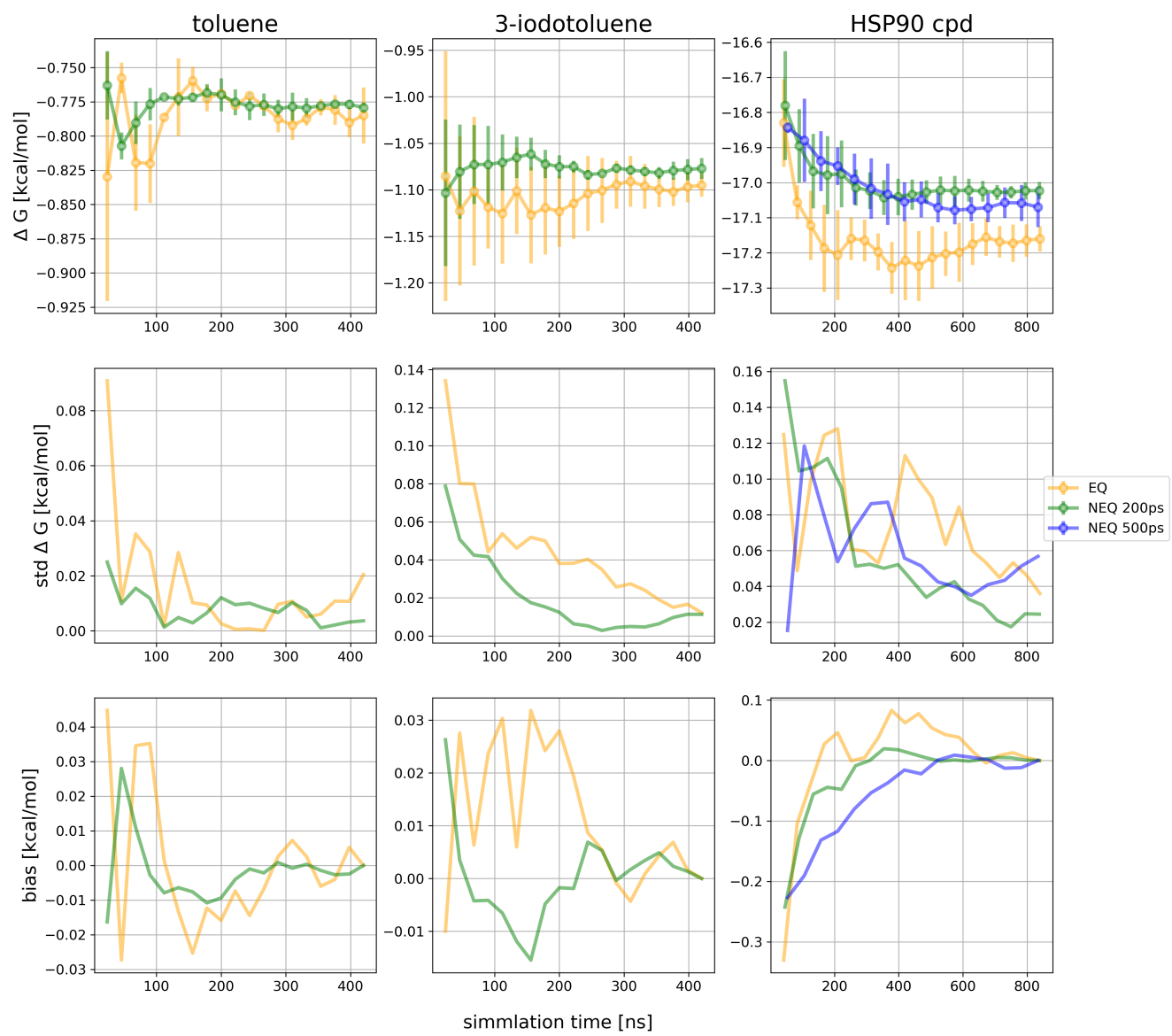

Figure S1. Hydration free energy, standard deviation (std) and bias as a function of total simulation time for toluene, 3-iodotoluene and the HSP90 compound. For the EQ approach (yellow) we show the mean free energy difference across three replicates in the top row while for the NEQ approach (green and blue) the free energy estimate was obtained by pooling work values from three replicates and estimating one free energy difference using BAR (Sec. 4.5). The uncertainty estimate in both approaches is the standard deviation across three replicates. Second and third row represents the standard deviation and bias. For the bias we assumed that the final value of the mean free energy is the theoretical hydration free energy of the compounds, meaning that it may be underestimated if simulations did not converge. Standard deviation and bias were reduced faster with the NEQ approach, suggesting that it is slightly more efficient in this test set. For the HSP90 compound the two methods did not converge to the same mean free energy within uncertainty. We tested a different NEQ protocol with longer, but fewer transitions (blue, $500 \mathrm{ps} /$ transition). EQ and NEQ approaches converged to statistically the same result using this protocol. 


\section{Restraints in binding free energy calculations}

Table 2. Protein (P1, P2, P3) and ligand atoms (L1, L2, L3) involved in Boresch-style restraints. One distance (P3-L1), two angles (P2-P3-L1 and P3-L1-L2) and three dihedrals (P1-P2-P3-L1, P2-P3-L1-L2 and P3-L1-L2-L3) were restrained to their value in an equilibrated reference structure.

\begin{tabular}{|l|l|l|l|l|l|l|}
\hline & P1 & P2 & P3 & L1 & L2 & L3 \\
\hline \hline $\begin{array}{l}\text { toluene Pose } \\
\text { I }\end{array}$ & Cys54 CA & Ala98 O & Ala99 O & C3 & C5 & C4 \\
\hline $\begin{array}{l}\text { toluene Pose } \\
\text { II }\end{array}$ & Cys54 CA & Ala98 O & Ala99 O & C3 & C5 & C4 \\
\hline $\begin{array}{l}3- \\
\text { iodotoluene }\end{array}$ & Cys54 CA & Ala98 O & Ala99 O & C1 & C6 & C3 \\
\hline $\begin{array}{l}\text { HSP90 com- } \\
\text { pound }\end{array}$ & Tyr201 CD2 & Pro164 C & Gly82 O & N2 & C1 & C3 \\
\hline
\end{tabular}




\section{Toluene binding to T4 lysozyme L99A}

Table 3. Binding free energy $\Delta G^{\circ}$ for the toluene/T4 lysozyme system. We show the free energy difference of each individual leg of the thermodynamic cycle as well as the binding free energy calculated by summation along the cycle. Reported uncertainties for decoupling the ligand in the binding site $\left(\Delta G_{\text {decouple ligand }}\right)$ and in the solvent $\left(\Delta G_{\text {solvate ligand }}\right)$ are the standard deviation of three independent replicates. $\Delta G_{\text {restraints of } f \text { was calculated }}$ analytically, using the formula in Boresch et al. ${ }^{52}$

\begin{tabular}{|c|c|c|c|c|c|}
\hline & $\begin{array}{l}\Delta G_{\text {restraints on }} \\
{[\mathrm{kcal} / \mathrm{mol}]}\end{array}$ & $\begin{array}{l}\Delta G_{\text {decouple ligand }} \\
{[\mathrm{kcal} / \mathrm{mol}]}\end{array}$ & \begin{tabular}{|l|}
$\Delta G_{\text {restraints }}$ of \\
{$[\mathrm{kcal} / \mathrm{mol}]$}
\end{tabular} & $\begin{array}{l}\Delta G_{\text {solvate ligand }} \\
{[\mathrm{kcal} / \mathrm{mol}]}\end{array}$ & $\begin{array}{l}\Delta G^{0} \\
{[\mathrm{kcal} / \mathrm{mol}]}\end{array}$ \\
\hline Pose I EQ & \multirow{4}{*}{$1.96 \pm 0.08$} & $11.68 \pm 0.08$ & \multirow{4}{*}{-8.615} & $-0.79 \pm 0.02$ & $-4.2 \pm 0.1$ \\
\hline $\begin{array}{l}\text { Pose I NEQ } \\
200 \mathrm{ps}\end{array}$ & & $11.6 \pm 0.3$ & & \multirow[t]{3}{*}{$-0.779 \pm 0.004$} & $-4.2 \pm 0.3$ \\
\hline $\begin{array}{l}\text { Pose I NEQ } \\
500 \mathrm{ps}\end{array}$ & & $11.7 \pm 0.3$ & & & $-4.3 \pm 0.3$ \\
\hline $\begin{array}{l}\text { Pose I NEQ } \\
1 \mathrm{~ns}\end{array}$ & & $11.7 \pm 0.2$ & & & $-4.3 \pm 0.2$ \\
\hline Pose II EQ & \multirow{4}{*}{$1.3 \pm 0.1$} & $9.6 \pm 0.1$ & \multirow{4}{*}{-6.774} & $-0.79 \pm 0.02$ & $-3.3 \pm 0.1$ \\
\hline $\begin{array}{l}\text { Pose II NEQ } \\
200 \mathrm{ps}\end{array}$ & & $9.6 \pm 0.1$ & & \multirow{3}{*}{$-0.779 \pm 0.004$} & $-3.3 \pm 0.1$ \\
\hline $\begin{array}{l}\text { Pose II NEQ } \\
500 \mathrm{ps}\end{array}$ & & $9.51 \pm 0.08$ & & & $-3.3 \pm 0.1$ \\
\hline $\begin{array}{l}\text { Pose II NEQ } \\
1 \mathrm{~ns}\end{array}$ & & $9.5 \pm 0.2$ & & & $-3.2 \pm 0.2$ \\
\hline
\end{tabular}

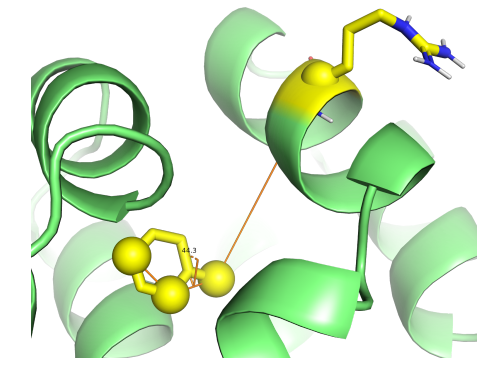

(a)
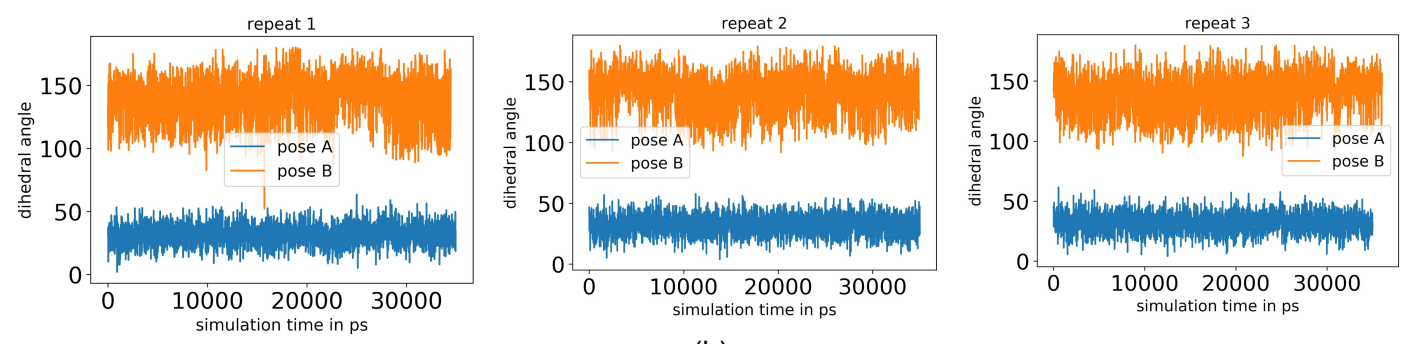

(b)

Figure S2. Two binding modes of toluene in T4 lysozyme L99A. (a) The dihedral angle used to differentiate the two bindings modes is defined by the C1, C3 and C5 atoms of toluene and the alpha carbon of arginine 119, depicted as spheres. (b) The crystallographic binding mode (pose I) is shown in blue and the alternative mode (pose II) in orange. Orientational restraints kept toluene from transitioning between binding modes. The two binding modes were restrained with different force constants. Pose I was restrained with a force constant of $20 \mathrm{kcal} /\left(\mathrm{mol} * \AA^{2}\right)$ for the bond and $20 \mathrm{kcal} /\left(\mathrm{mol} * \mathrm{rad}^{2}\right)$ for angles and dihedrals, while the alternative binding mode was restrained with $10 \mathrm{kcal} /\left(\mathrm{mol} * \AA^{2}\right)$ and $10 \mathrm{kcal} /\left(\mathrm{mol} * \mathrm{rad}^{2}\right)$. 
repeat 1
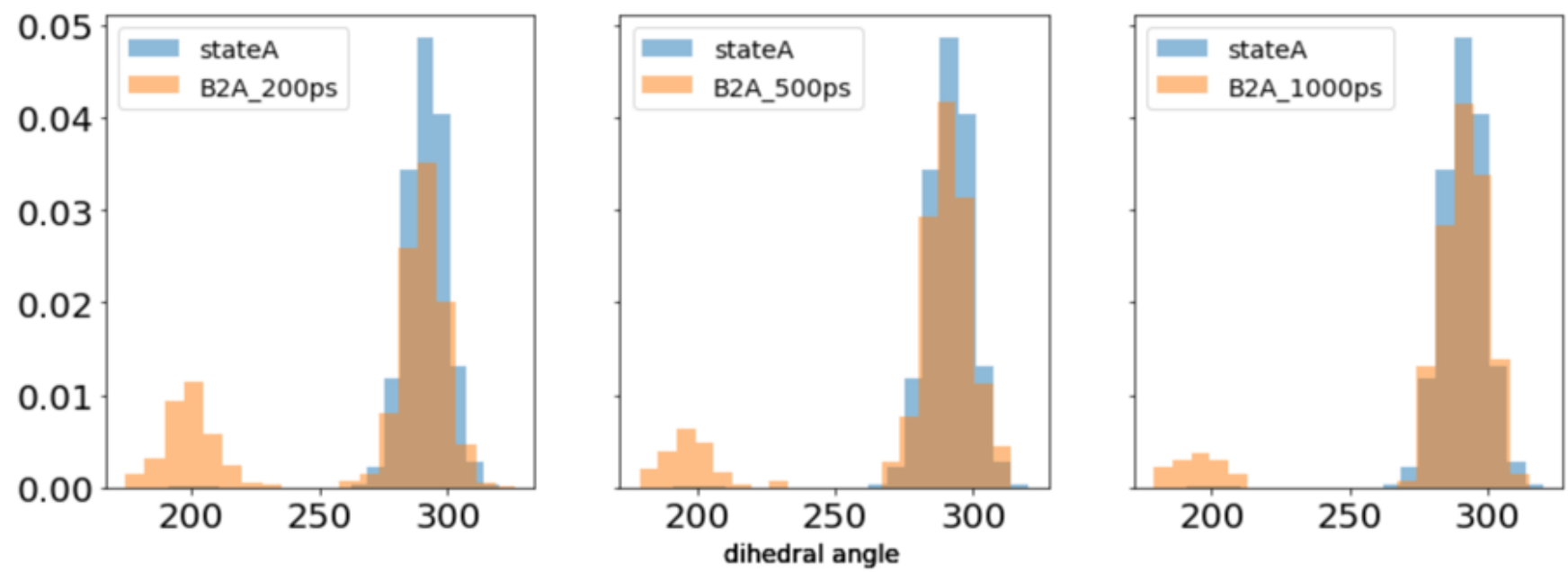

repeat 1
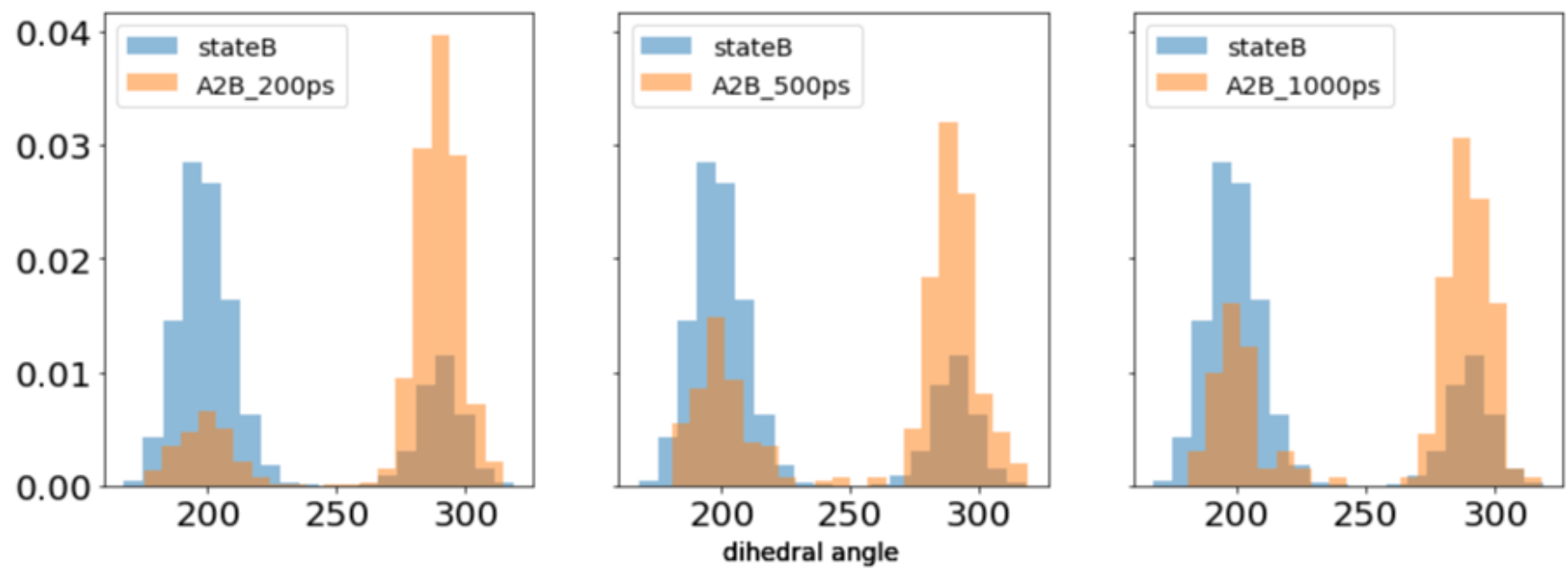

Figure S3. The distribution of the dihedral angle $\xi 1$ of Ile78 for toluene binding to T4 lysozyme L99A. Shown are values in the end states (blue) and the last frame of the switching transitions towards the end state (orange; A2B means going from state A to state B) for different transition lengths. Especially for the decoupling transitions (bottom, A2B: interacting to non-interacting), the transition length had an impact on how many transitions reoriented to the other Ile78 rotamer. This figure shows only one repeat in the crystallographic pose, all repeats and the two poses have very similar distributions and can be found in the SI. 


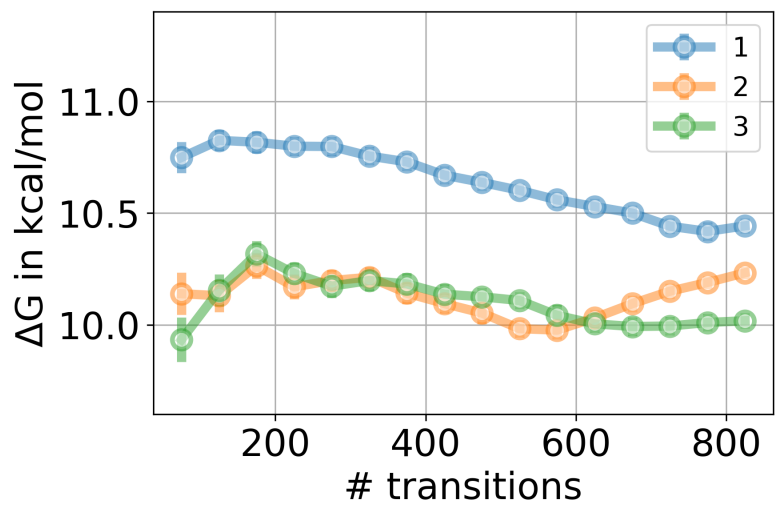

(a)

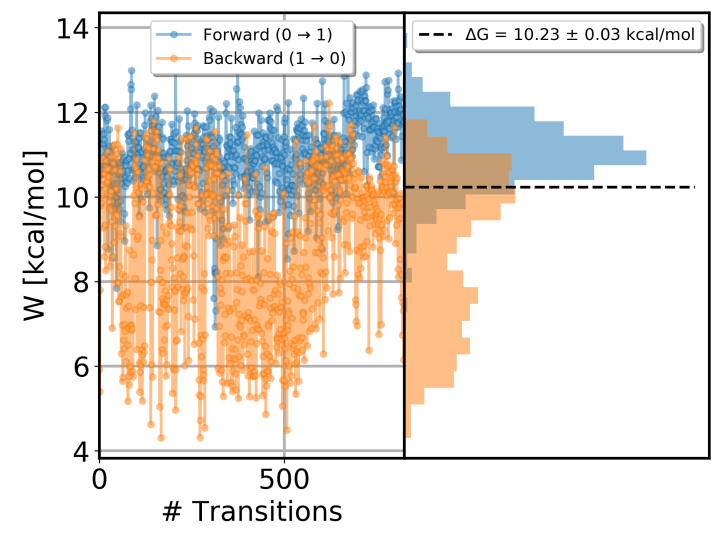

(b)

Figure S4. Protocol using weaker Boresch-style restraints $\left(5 \mathrm{kcal} /\left(\mathrm{mol} * \AA^{2}\right)\right.$ and $\left.5 \mathrm{kcal} /\left(\mathrm{mol} * \mathrm{rad}^{2}\right)\right)$. (a) Free energy difference for decoupling toluene in the binding site with the NEQ protocol using weaker Boreschstyle restraints. The cumulative free energy difference is plotted against the number of transitions. The standard deviation across three independent replicates is $0.2 \mathrm{kcal} / \mathrm{mol}$ which is slightly lower than in the protocol with higher force constant on the restraints $(0.3 \mathrm{kcal} / \mathrm{mol})$. (b) Work values for toluene binding to T4 lysozyme L99A. Shown are values measured for each attempted transition, in forward (blue) and reverse (orange) direction, as well as the distribution of the work values. The reverse work distribution is similar to the one in the protocol using stronger restraints and the mean dissipation was independent of the restraint strength and in both protocols $1.7 \mathrm{kcal} / \mathrm{mol}$ in the reverse direction and $0.9 \mathrm{kcal} / \mathrm{mol}$ in the forward direction.

Table 4. Comparison of the free energy difference for decoupling toluene in T4 lysozyme L99A using Boresch-style restraints and center-of-mass $(\mathrm{COM})$ - COM flat-bottom harmonic distance restraints. For the COM-COM restraint protocol we first turned the Boresch-style restraints off while turning on a distance restraint between the COM of the ligand and the COM of the side chain alanine 99. Then we decoupled the ligand and afterwards switched the COM-COM restraint back to the Boresch-style restraints in the noninteracting state. Reported uncertainties are the standard deviation of three independent replicates. In the NEQ approach, the dissipated work was higher in the COM-COM restraint protocol $(3.8 \mathrm{kcal} / \mathrm{mol}$ in the reverse direction and 1.4 in the forward direction) than in the protocol using Boresch-style restraints. With a single COM-COM distance restraint, the ligand can sample the sphere around the radius of that distance restraint which presumably caused the larger dissipation.

\begin{tabular}{|c|c|c|c|c|c|}
\hline & & $\begin{array}{l}\Delta G_{C O M} \text { on, Boresch off } \\
{[\mathrm{kcal} / \mathrm{mol}]}\end{array}$ & $\begin{array}{l}\Delta G_{\text {decouple ligand }} \\
{[\mathrm{kcal} / \mathrm{mol}]}\end{array}$ & $\begin{array}{l}\Delta G_{C O M} \text { off }, \text { Boresch on } \\
{[\mathrm{kcal} / \mathrm{mol}]}\end{array}$ & $\begin{array}{l}\Delta G_{\text {total }} \\
{[\mathrm{kcal} / \mathrm{mol}]}\end{array}$ \\
\hline $\begin{array}{l}\text { Only } \\
\text { Boresch } \\
\text { restraints }\end{array}$ & NEQ & & $11.6 \pm 0.3$ & & $11.6 \pm 0.3$ \\
\hline \multirow{2}{*}{$\begin{array}{l}\text { COM-COM } \\
\text { distance } \\
\text { restraints }\end{array}$} & EQ & \multirow[t]{2}{*}{$-1.87 \pm 0.03$} & $5.9 \pm 0.1$ & \multirow[t]{2}{*}{$8.38 \pm 0.04$} & $12.4 \pm 0.1$ \\
\hline & NEQ & & $5.9 \pm 0.3$ & & $12.4 \pm 0.3$ \\
\hline
\end{tabular}



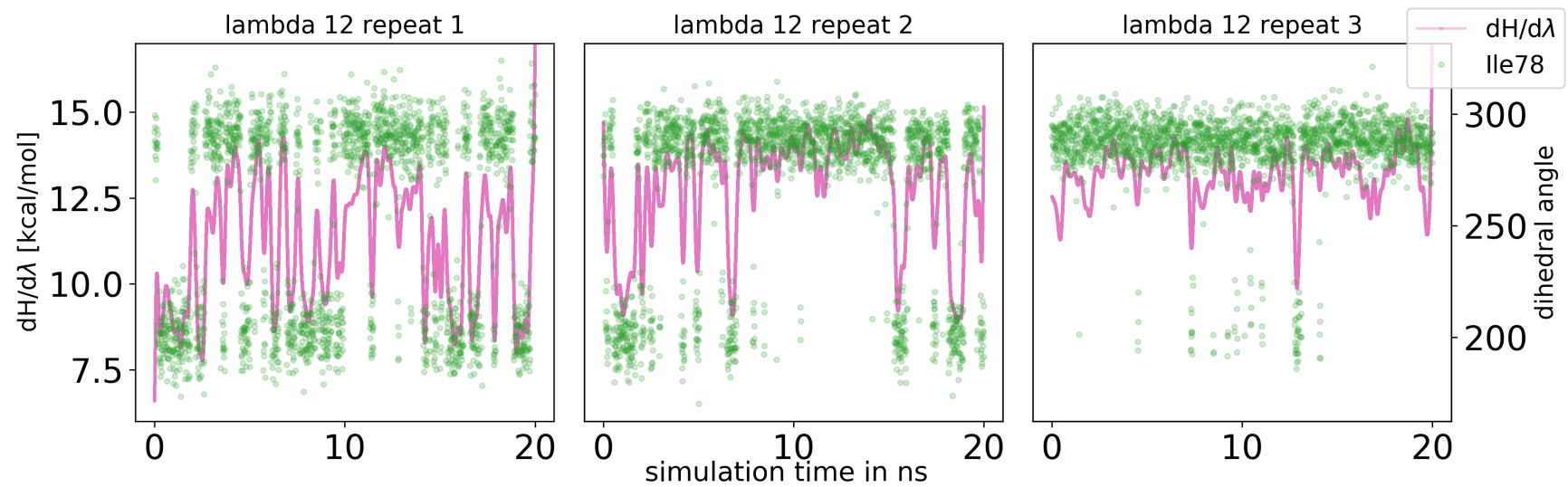

Figure S5. Correlation between $\mathrm{dH} / \mathrm{d} \lambda$ values and the dihedral angle of Ile78 for toluene binding to T4 lysozyme L99A. A running average of the $\mathrm{dH} / \mathrm{d} \lambda$ values (pink, averaged across 2000 data points) and the dihedral angle of Ile78 (green) is plotted as a function of simulation time. Here we only show $\lambda 12$ for the three independent replicates, additional $\lambda$ windows can be found in the SI. Sudden changes in $\mathrm{dH} / \mathrm{d} \lambda$ correlated with rotation of the Ile78 side chain. 


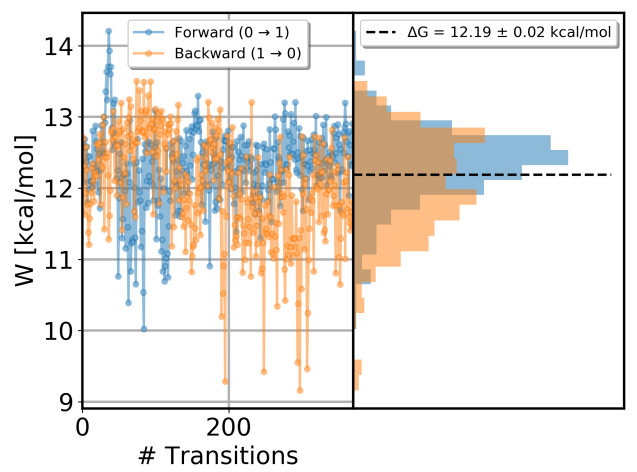

(a)

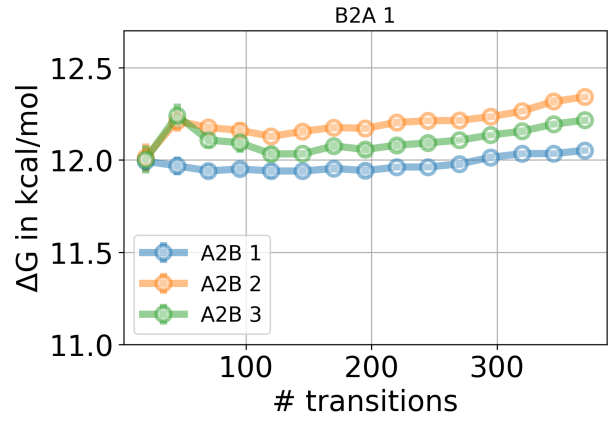

(c)

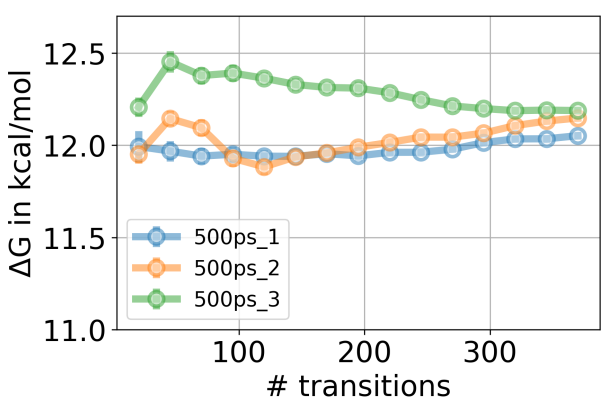

(b)

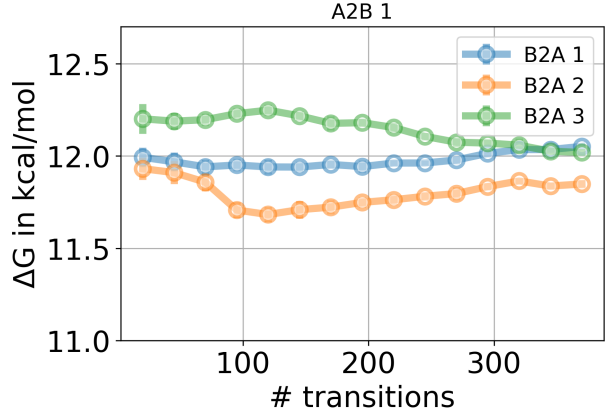

(d)

Figure S6. Restraining the Ile78 side chain in the NEQ protocol to further investigate its impact. (a) Work values for toluene binding to T4 lysozyme L99A while restraining the Ile78 side chain. Shown are values measured for each attempted transition, in forward (blue) and reverse (orange) direction, as well as the distribution of the work values. In contrast to the unrestrained protocol, the reverse work distribution (orange) is unimodal, which suggests that sampling of two Ile78 rotamers caused the bimodal work distributions in the unrestrained simulations. (b) Free energy difference for decoupling toluene in the binding site with the NEQ protocol using dihedral restraints. The cumulative free energy difference is plotted against the number of transitions. Three independent replicates seem to converge to the same free energy difference and the standard deviation was lower in this protocol compared to the unrestrained calculations (0.06 vs. $0.3 \mathrm{kcal} / \mathrm{mol})$.(c) and (d) Analyzing the set of forward and reverse data of replicates separately. Three replicates give three sets of transitions in the forward direction and reverse direction which are independent from one another. We took a fixed set of transitions from one replicate for one direction (in (c) the set of transitions from the first replicate was used for the reverse direction (B2A 1)) and calculated the free energy difference using data from the other direction of all three replicates (in (c) A2B 1 in blue uses transitions in the forward direction from replicate one). The same was performed for the other direction in (d). The free energy difference of the second replicate (orange) is higher in the forward direction (c) and lower in the reverse direction (d). This shows that the apparent convergence (and low standard deviation) seen in (b) is likely a coincidence. 


\section{3-Iodotoluene binding to T4 lysozyme L99A}

Table 5. Binding free energy $\Delta G^{\circ}$ for the 3-iodotoluene/T4 lysozyme system. We show the free energy difference of each individual leg of the thermodynamic cycle as well as the binding free energy calculated by summation along the cycle. Reported uncertainties are the standard deviation of three independent replicates. $\Delta G_{\text {restraints off }}$ was calculated analytically, using the formula in Boresch et al. ${ }^{52}$ Costs for restraining and releasing the restraints on Val111 were 0.1 and $-0.1 \mathrm{kcal} / \mathrm{mol}$.

\begin{tabular}{|c|c|c|c|c|c|}
\hline & $\begin{array}{l}\Delta G_{\text {restraints on }} \\
{[\mathrm{kcal} / \mathrm{mol}]}\end{array}$ & $\begin{array}{l}\Delta G_{\text {decouple ligand }} \\
{[\mathrm{kcal} / \mathrm{mol}]}\end{array}$ & $\begin{array}{l}\Delta G_{\text {restraints off }} \\
{[\mathrm{kcal} / \mathrm{mol}]}\end{array}$ & $\begin{array}{l}\Delta G_{\text {solvate ligand }} \\
{[\mathrm{kcal} / \mathrm{mol}]}\end{array}$ & $\begin{array}{l}\Delta G^{0} \\
{[\mathrm{kcal} / \mathrm{mol}]}\end{array}$ \\
\hline EQ & \multirow{7}{*}{$1.7 \pm 0.1$} & $12.8 \pm 0.2$ & \multirow{7}{*}{-8.638} & $-1.10 \pm 0.01$ & $-4.8 \pm 0.2$ \\
\hline NEQ $500 \mathrm{ps}$ & & $13.6 \pm 0.2$ & & \multirow{4}{*}{$-1.08 \pm 0.01$} & $-5.6 \pm 0.2$ \\
\hline NEQ $1 \mathrm{~ns}$ & & $13.7 \pm 0.1$ & & & $-5.7 \pm 0.1$ \\
\hline NEQ 2 ns & & $13.5 \pm 0.3$ & & & $-5.5 \pm 0.3$ \\
\hline NEQ 4 ns & & $13.1 \pm 0.1$ & & & $-5.1 \pm 0.1$ \\
\hline $\begin{array}{l}\text { restrain } \\
\text { Val111 EQ }\end{array}$ & & $13.0 \pm 0.4$ & & $-1.10 \pm 0.01$ & $-5.0 \pm 0.4$ \\
\hline $\begin{array}{l}\text { restrain } \\
\text { Val111 NEQ }\end{array}$ & & $12.77 \pm 0.07$ & & $-1.08 \pm 0.01$ & $-4.8 \pm 0.1$ \\
\hline
\end{tabular}
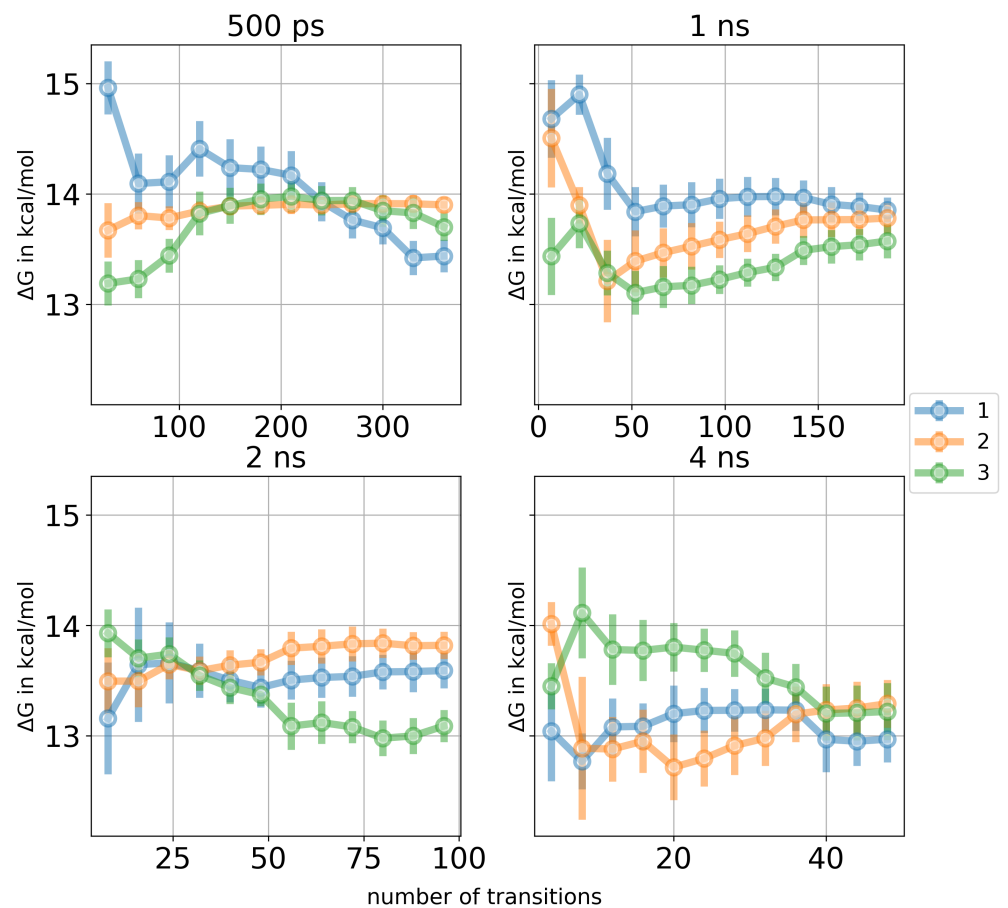

Figure S7. Free energy difference for decoupling 3-iodotoluene in the binding site using non-equilibrium FEC. The cumulative free energy difference is plotted as a function of the number of transitions. We tested four different protocols that differ in the length of the non-equilibrium transition and the number of transitions. Three independent replicates (blue, orange, green) were run for each protocol. Uncertainties were estimated via bootstrapping. The different protocols did not converge to the same free energy difference in the simulation time. 


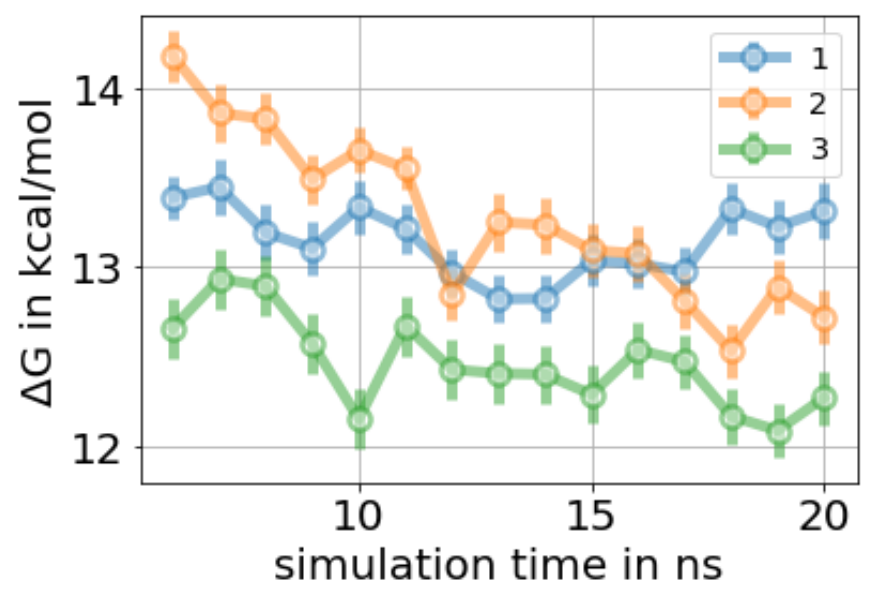

Figure S8. Free energy difference for decoupling 3-iodotoluene in the binding site using equilibrium FEC. The cumulative free energy difference of three independent replicates is plotted as a function of the simulation time per $\lambda$ window. An analytical uncertainty estimate was used. Independent replicates did not converge to the same free energy difference within uncertainty in $20 \mathrm{~ns}$ per $\lambda$ window simulation time.
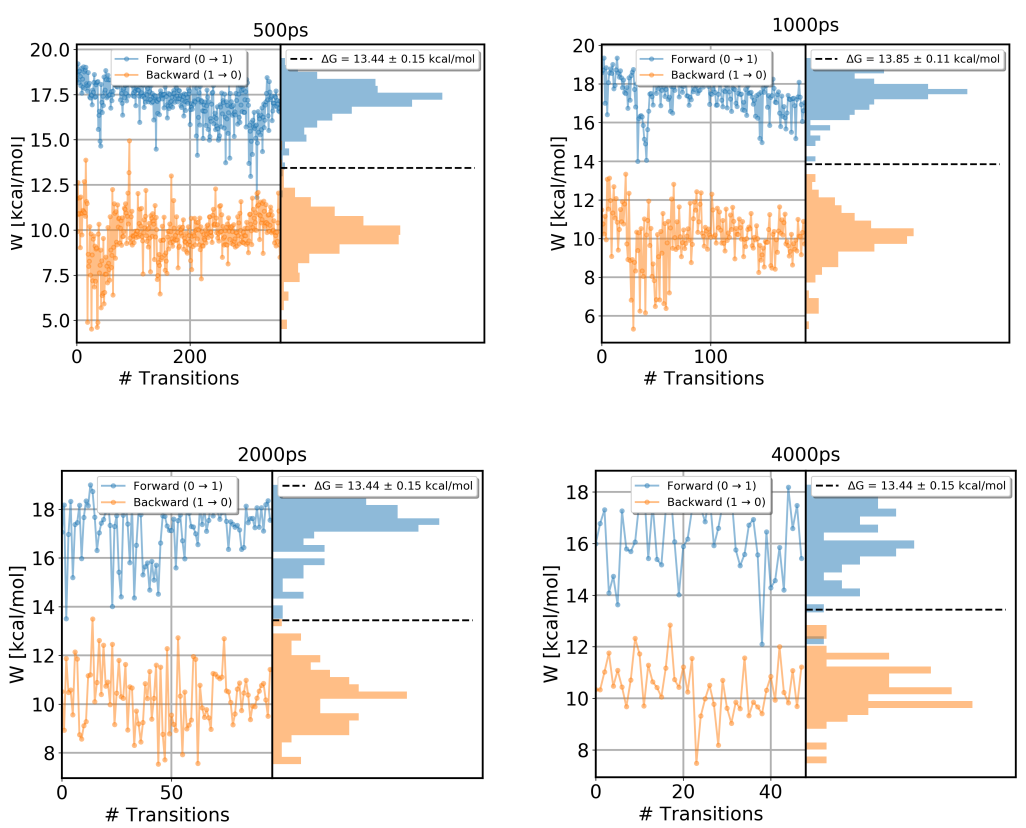

Figure S9. Work values for 3-iodotoluene binding to T4 lysozyme L99A, in forward (blue) and reverse (orange) direction. Shown are values measured for each attempted transition, as well as the distribution of the work values. The four plots show protocols that differ in the length of each NEQ switching transitions. The overlap of the work distributions in all protocols was poor, however improved slightly with transition length. 


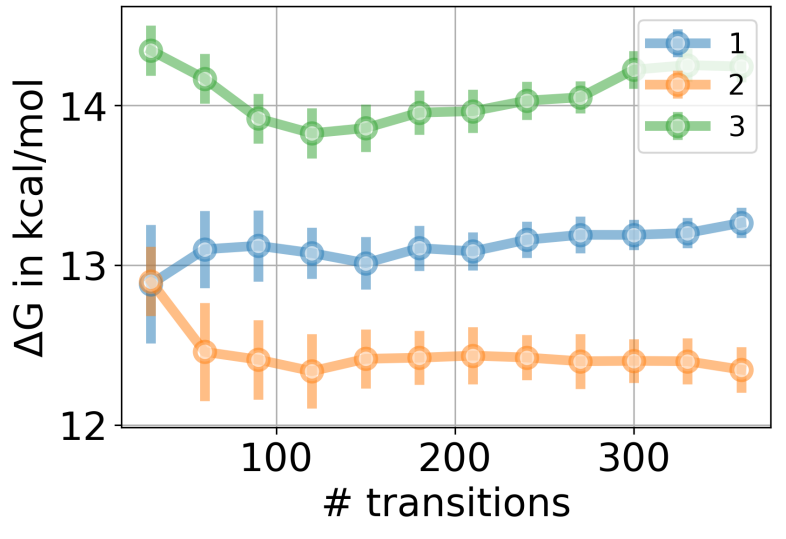

(a)

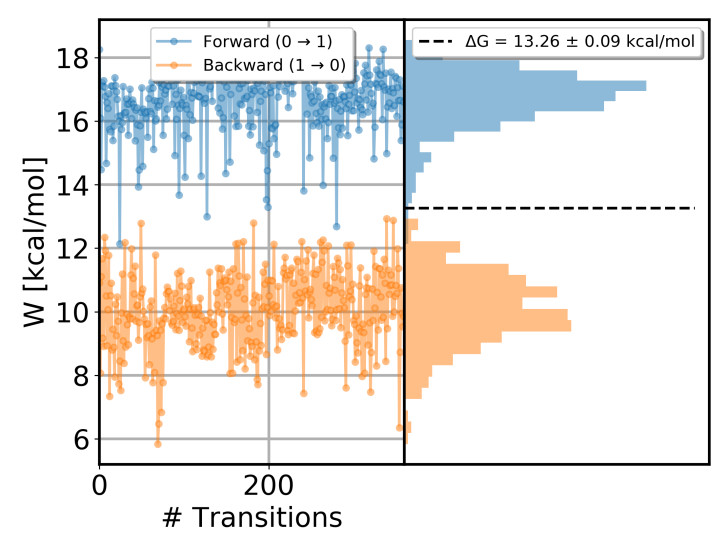

(b)

Figure S10. Weaker Boresch-style restraints $\left(5 \mathrm{kcal} /\left(\mathrm{mol} * \AA^{2}\right)\right.$ and $\left.5 \mathrm{kcal} /\left(\mathrm{mol} * \mathrm{rad}^{2}\right)\right)$. (a) Free energy difference for decoupling 3-iodotoluene in the binding site with the NEQ protocol using weaker Boresch-style restraints. The cumulative free energy difference is plotted against the number of transitions. The standard deviation across three independent replicates was $0.8 \mathrm{kcal} / \mathrm{mol}$ which is higher than in the protocol with stronger force constant on the restraints $(0.2 \mathrm{kcal} / \mathrm{mol})$. (b) Work values for 3-iodotoluene binding to T4 lysozyme L99A. Shown are values for replicate 1 measured for each attempted transition, in forward (blue) and reverse (orange) direction, as well as the distribution of the work values. The reverse work distribution is similar to the one in the protocol using stronger restraints and the mean dissipation was similar in both protocols, in the reverse direction $3.7 \mathrm{kcal} / \mathrm{mol}$ for the protocol with a weaker force constant and $3.9 \mathrm{kcal} / \mathrm{mol}$ for the other protocol and in the forward direction, $3.4 \mathrm{vs.}$ $4.1 \mathrm{kcal} / \mathrm{mol}$, respectively.

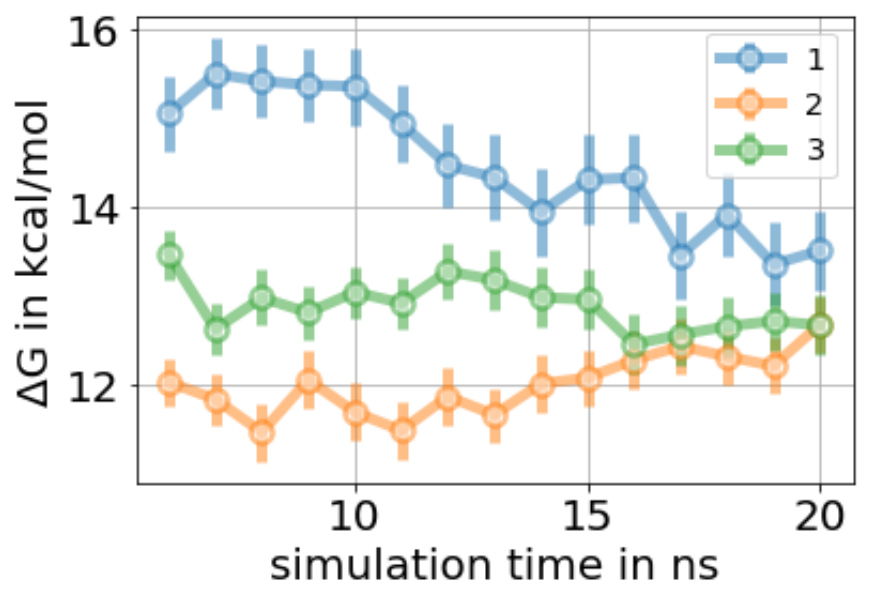

Figure S11. Decoupling 3-iodotoluene in the binding site while switching the Val111 side chain using the EQ approach. We show the free energy difference of three replicates as a function of simulation time. 


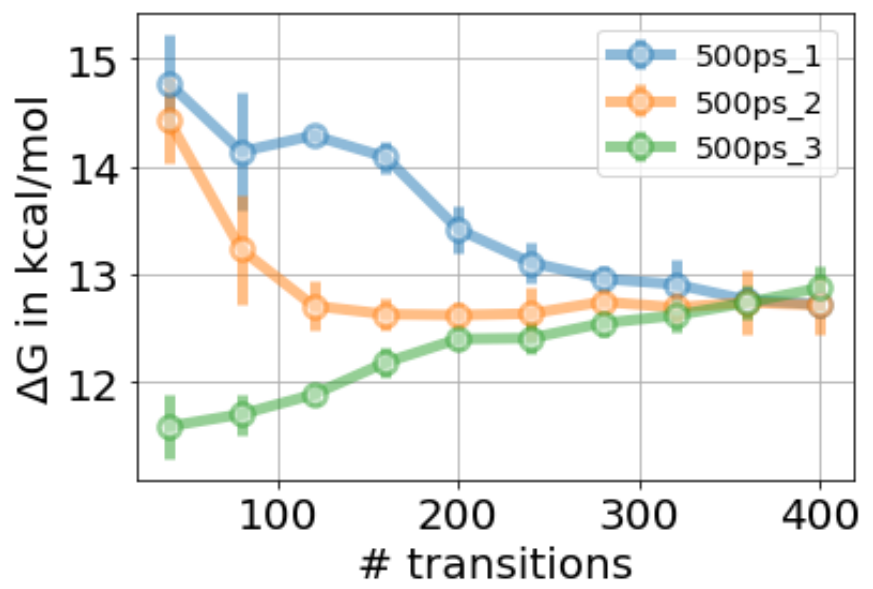

Figure S12. Decoupling 3-iodotoluene in the binding site while switching the Val111 side chain using the NEQ approach. We show the free energy difference of three replicates as a function of number of transitions. The $\Delta G$ estimate converged approximately after 320 transitions. 


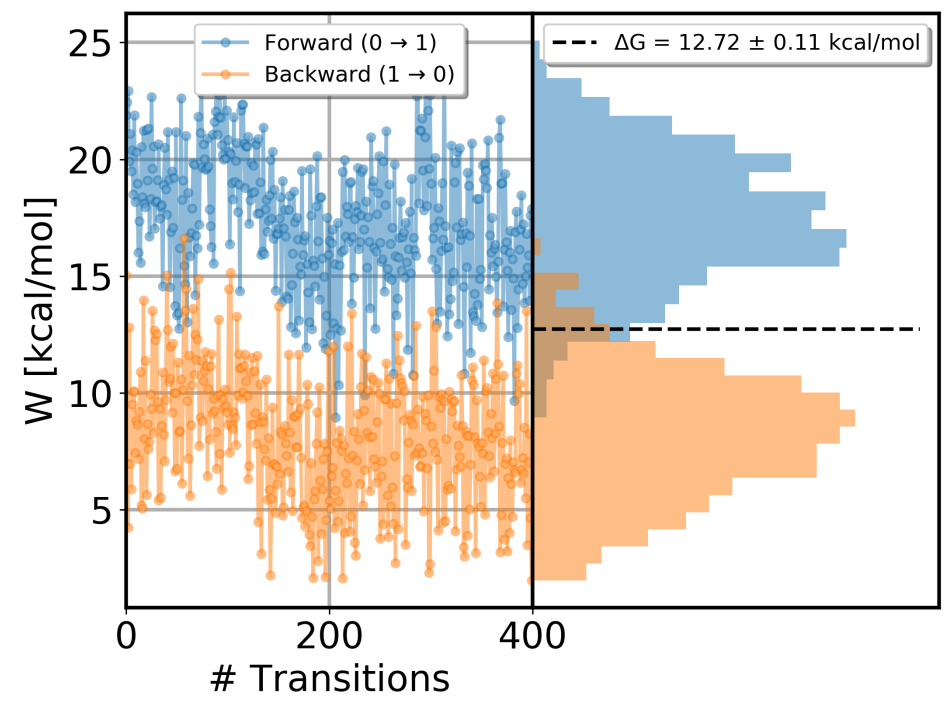

Figure S13. Work values and work distributions for decoupling 3-iodotoluene in the binding site of T4 lysozyme L99A while switching the Val111 side chain. Work values of the forward direction (decoupling, blue) and reverse direction (coupling, orange) are plotted as a function of transition number and the distribution of work values in the vertical plot. Transitions were run for $500 \mathrm{ps}$ each. Forward and reverse work distributions overlapped well when the side chain was switched during the alchemical path, compared to the unrestrained protocol (Figure S9, 500 ps).

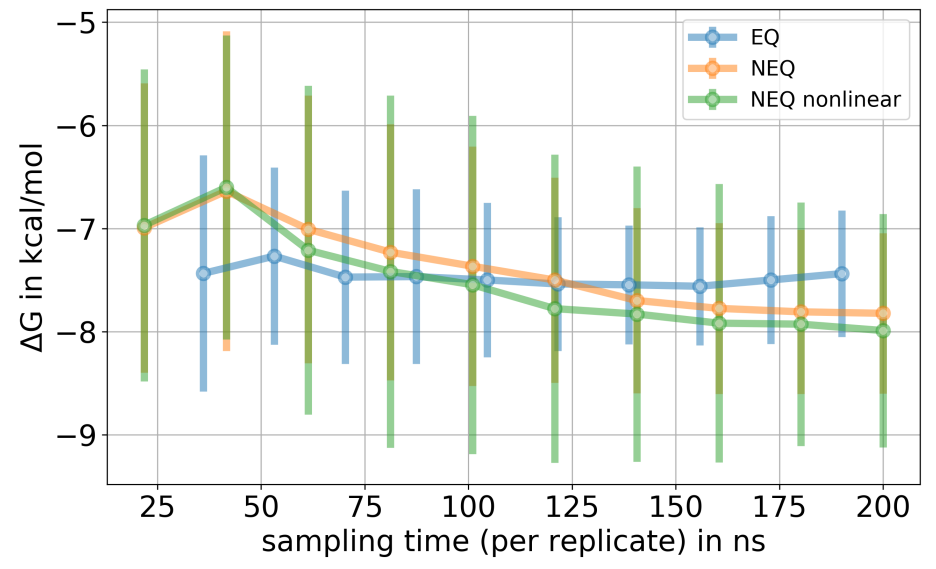

Figure S14. Free energy difference for releasing the dihedral restraints in the decoupled state of the 3-iodotoluene/ T4 lysozyme system in the protocol where multiple dihedrals were restrained. The alchemical path was nonlinear (blue and green curve) or linear (orange curve). For the EQ approach we show the mean free energy difference across six replicates while for the NEQ approach the free energy estimate was obtained by pooling work values from six replicates and estimating one free energy difference using BAR (Sec. 4.5). The uncertainty estimate in both approaches is the standard deviation across six replicates. All protocols converged to the same free energy difference within uncertainty. In the NEQ approach, a nonlinear alchemical path, where more sampling time can be spend in the more challenging part of the transformation (lower force constants) did not perform better than the protocol using a linear alchemical path. The standard deviation was high $(0.6-1.1 \mathrm{kcal} / \mathrm{mol})$ indicating sampling problems in this protocol where multiple additional side chains were restrained. Input topology files for the nonlinear NEQ protocol are provided in the SI files. 


\section{The HSP90 system}

Table 6. Binding free energy $\Delta G^{\circ}$ for the HSP90 system. We show the free energy difference of each individual leg of the thermodynamic cycle as well as the binding free energy calculated by summation along the cycle. Reported uncertainties are the standard deviation of three independent replicates. $\Delta G_{\text {restraints off }}$ was calculated analytically. ${ }^{52}$ The last two rows represent protocols, where all crystallographic water molecules were removed from the input structure, including three buried binding site waters.

\begin{tabular}{|c|c|c|c|c|c|}
\hline & $\begin{array}{l}\Delta G_{\text {restraints on }} \\
{[\mathrm{kcal} / \mathrm{mol}]}\end{array}$ & $\begin{array}{l}\Delta G_{\text {decouple ligand }} \\
{[\mathrm{kcal} / \mathrm{mol}]}\end{array}$ & $\begin{array}{l}\Delta G_{\text {restraints off }} \\
{[\mathrm{kcal} / \mathrm{mol}]}\end{array}$ & $\begin{array}{l}\Delta G_{\text {solvate ligand }} \\
{[\mathrm{kcal} / \mathrm{mol}]}\end{array}$ & $\begin{array}{l}\Delta G^{0} \\
{[\mathrm{kcal} / \mathrm{mol}]}\end{array}$ \\
\hline EQ & \multirow{5}{*}{$1.26 \pm 0.08$} & $36.7 \pm 0.3$ & \multirow{5}{*}{-9.003} & \multirow{2}{*}{$-17.16 \pm 0.04$} & $-11.8 \pm 0.3$ \\
\hline HREX & & $35.9 \pm 0.5$ & & & $-11.0 \pm 0.5$ \\
\hline NEQ $1 \mathrm{~ns}$ & & $36.3 \pm 0.4$ & & \multirow{3}{*}{$-17.02 \pm 0.02$} & $-11.5 \pm 0.4$ \\
\hline NEQ $5 \mathrm{~ns}$ & & $36.2 \pm 0.3$ & & & $-11.4 \pm 0.3$ \\
\hline NEQ $10 \mathrm{~ns}$ & & $36.2 \pm 0.5$ & & & $-11.4 \pm 0.5$ \\
\hline EQ no water & \multirow{2}{*}{$1.4 \pm 0.04$} & $33.3 \pm 1.3$ & \multirow{2}{*}{-8.924} & \multirow{2}{*}{$-17.16 \pm 0.04$} & $-8.6 \pm 1.3$ \\
\hline HREX no water & & $34.6 \pm 0.8$ & & & $-9.9 \pm 0.8$ \\
\hline
\end{tabular}

(a)
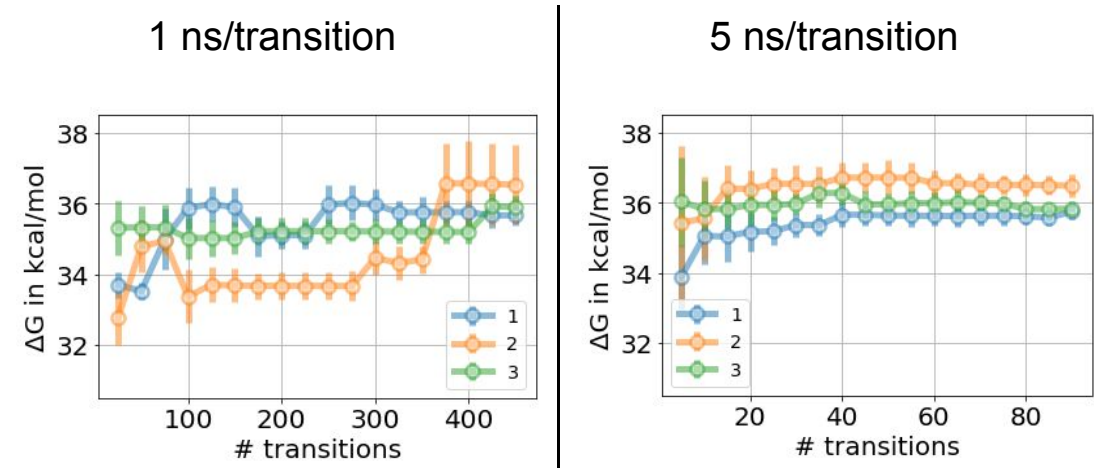

10 ns/transition

(b)
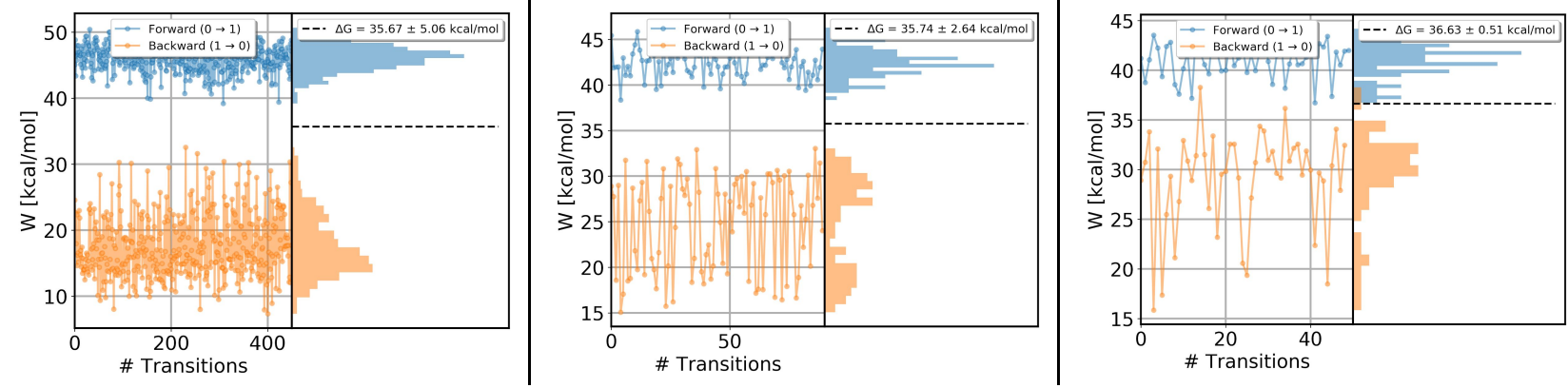

Figure S15. (a) Free energy difference of three independent replicates as a function of number of NEQ transitions in the HSP90 system. Three protocols using $1 \mathrm{~ns}, 5 \mathrm{~ns}$ or $10 \mathrm{~ns}$ per switching transition were used in the NEQ approach and uncertainty estimates obtained via bootstrapping. (b) Work values of forward (blue) and reverse (orange) transitions as a function of transition number and the distribution of work values. Shown is one example (replicate 1) per protocol, additional plots are provided in the SI. The uncertainty is the analytical error (see section 4.3). At poor overlap the uncertainty is high ( $5 \mathrm{kcal} / \mathrm{mol}$ for $1 \mathrm{~ns} /$ transition replicate 1$)$, indicating that the estimate is likely incorrect. 

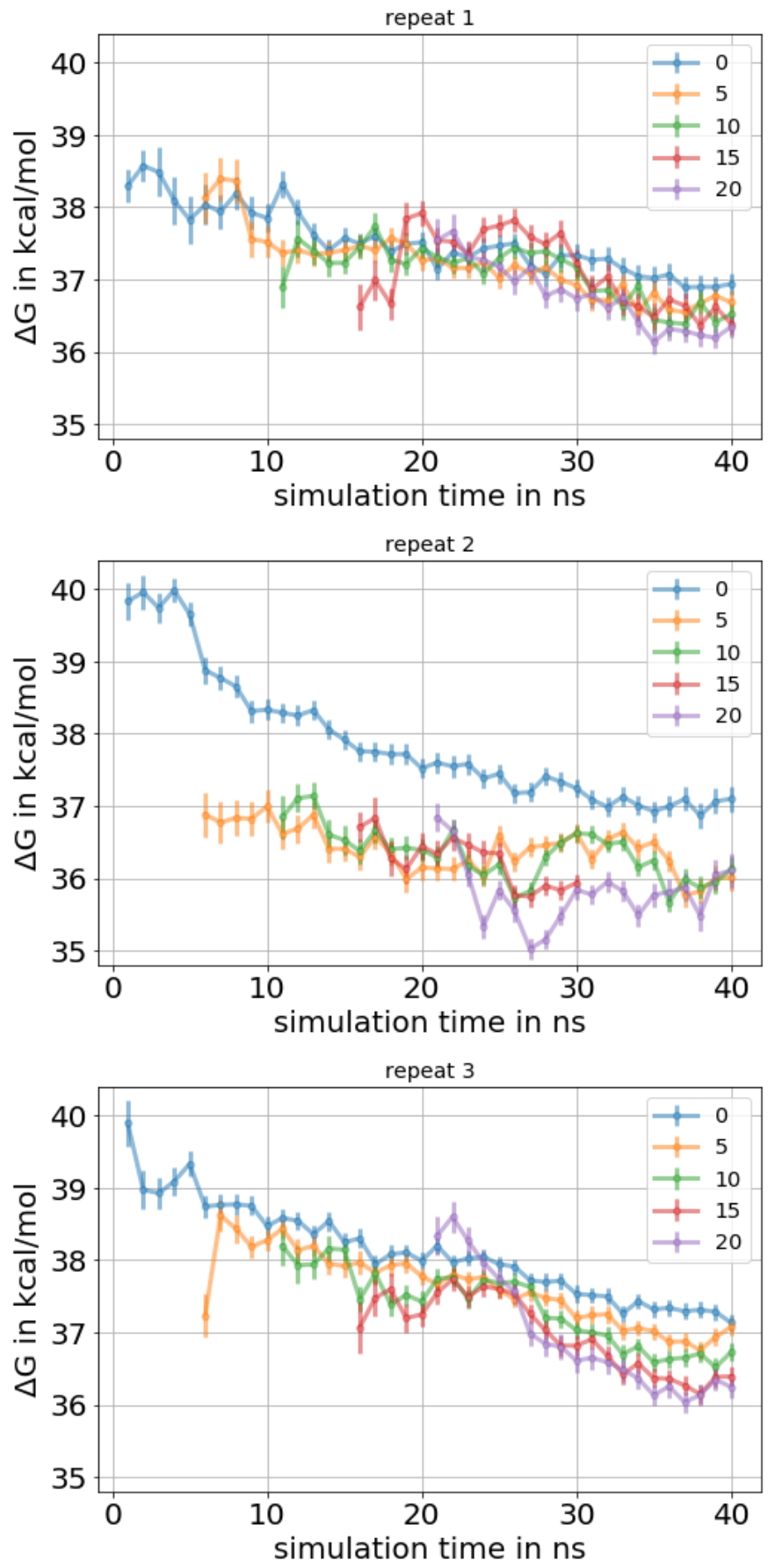

Figure S16. Impact of the equilibration time on the free energy difference in the HSP90 system. In a separate plot for every replicate, we show the cumulative free energy difference as a function of simulation time/lambda window. Different amount of data were discarded for equilibration, e.g. 0 means all data from the production run were used to estimate the free energy difference and 20 means $20 \mathrm{~ns} / \lambda$ window were discarded for equilibration. In all three repeats the final free energy difference depends on the amount of data discarded for equilibration indicating a slow DOF in the system. 


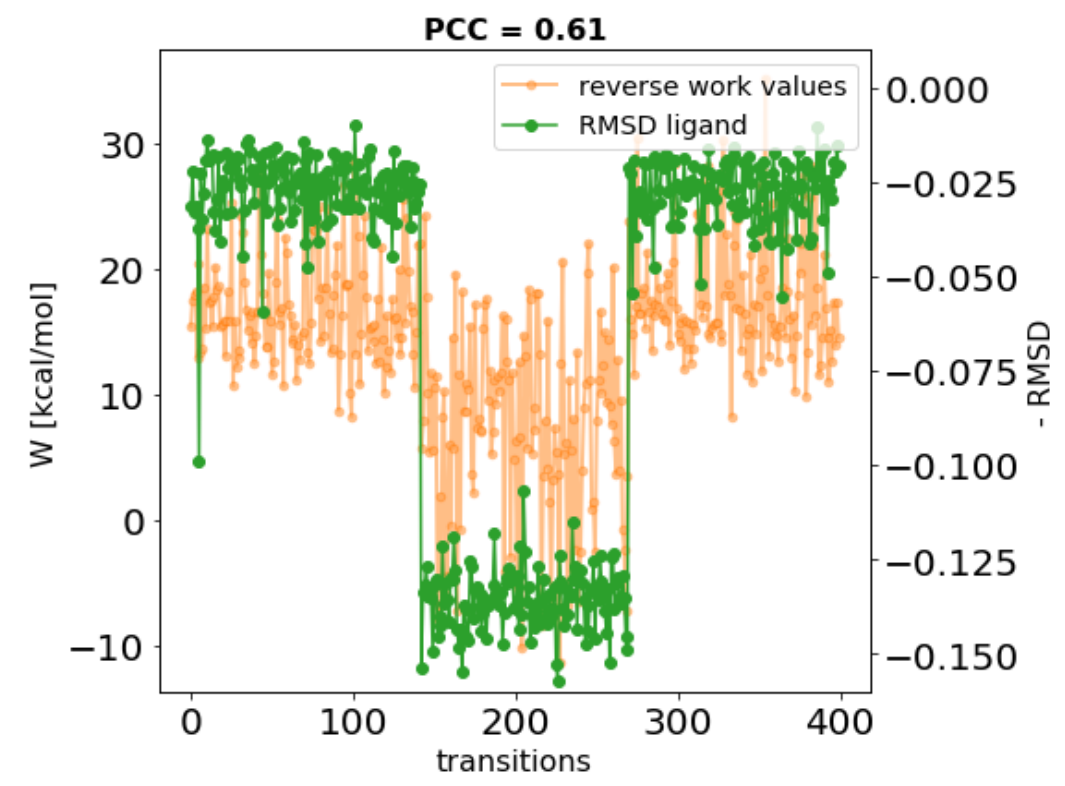

Figure S17. Correlation between reverse work values (Wr) and the ligand RMSD in the HSP90 system. The work values (orange) and the RMSD (green) are plotted as a function of the transition number. The RMSD was calculated from the set of first frames of the NEQ transitions. Work values correlated with the ligand RMSD, which is supported by a high Pearson correlation coefficient $(\mathrm{PCC}=0.61)$. The binding mode flip had an impact on the work values.

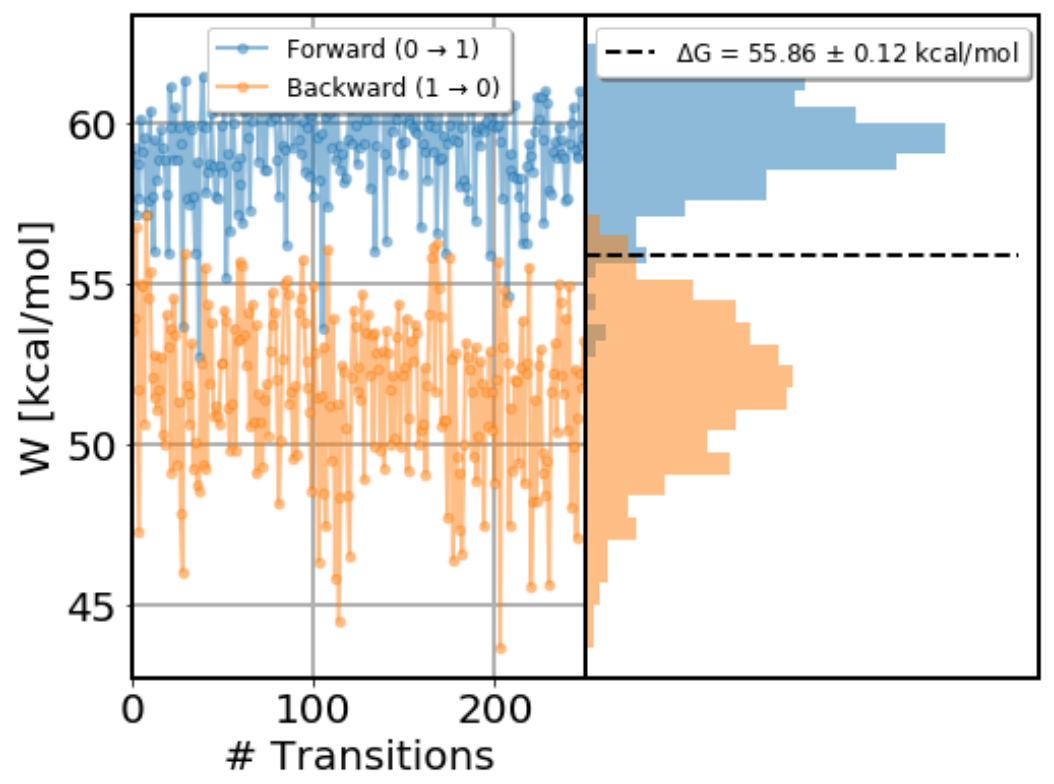

Figure S18. Work values for the ligand binding to HSP90, in forward (blue) and reverse (orange) direction in the presence of the bubble-ligand. Shown are values measured for each attempted transition, as well as the distribution of the work values. Transitions were run for $1 \mathrm{~ns}$ each. Forward and reverse work distributions overlapped well in the presence of the bubble-ligand, supporting that the poor overlap was caused by inadequate water sampling. 


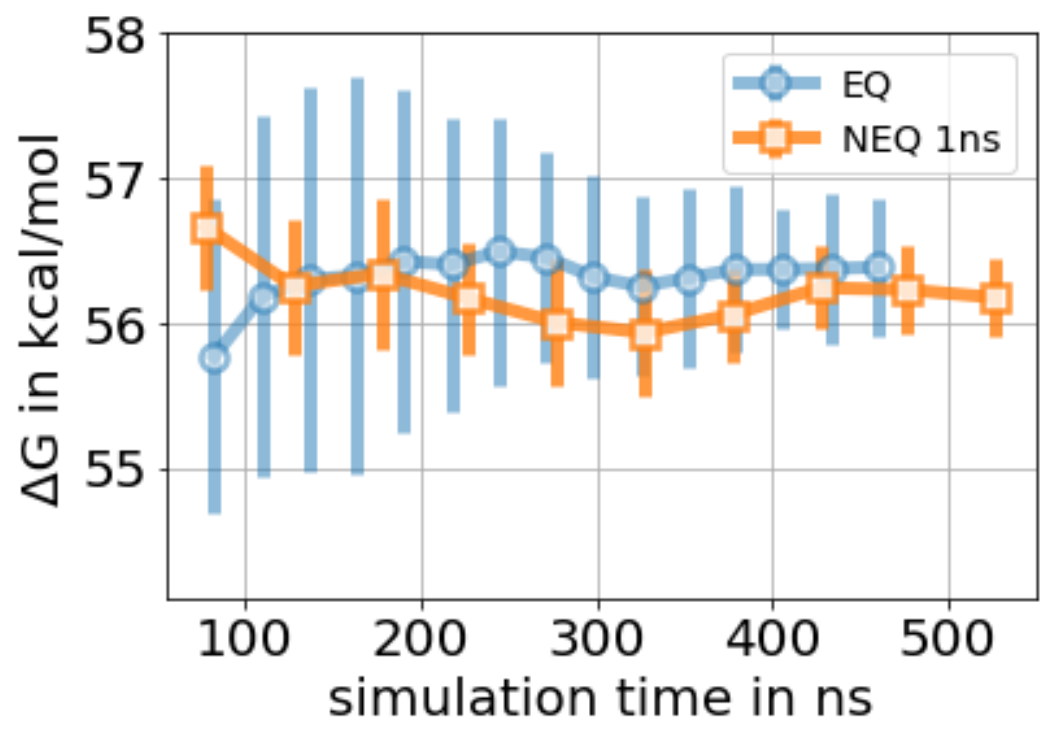

Figure S19. Free energy difference for decoupling the HSP90 ligand in the binding site in the presence of the bubble-ligand. For the EQ approach we show the mean free energy difference across three replicates while for the NEQ approach the free energy estimate was obtained by pooling work values from three replicates and estimating one free energy difference using BAR (Sec. 4.5). The uncertainty estimate in both approaches is the standard deviation across three replicates.
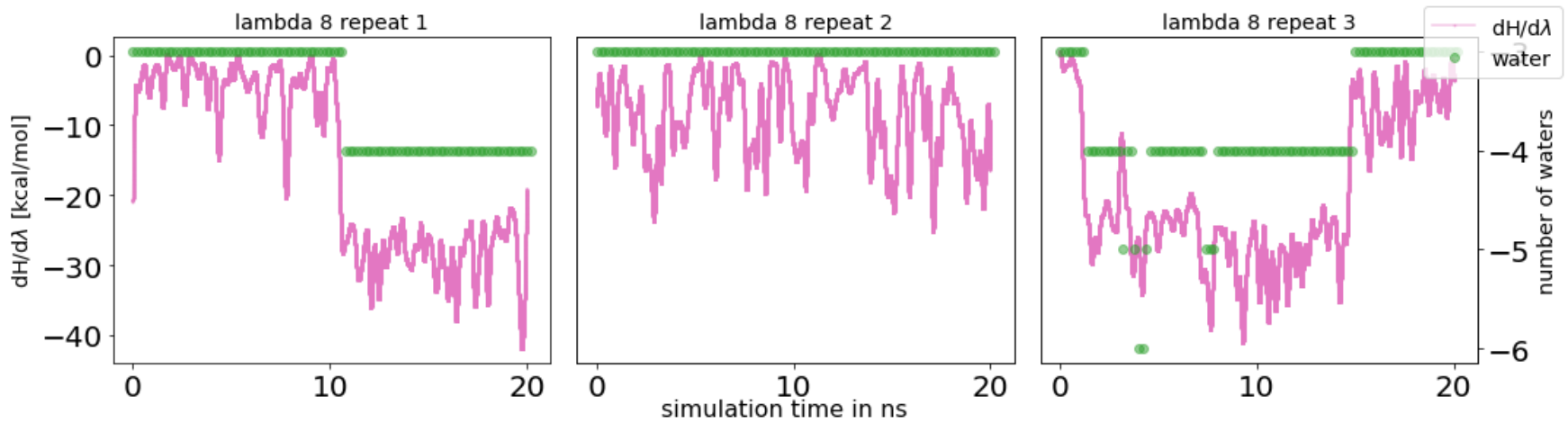

Figure S20. Water sampling problems when turning the interactions of the bubble-ligand off. Correlation between $\mathrm{dH} / \mathrm{d} \lambda$ values and number of water molecules in the binding site for the ligand binding to HSP90. A running average of the $\mathrm{dH} / \mathrm{d} \lambda$ values (pink, averaged across 2000 data points) and the number of water molecules (green) is plotted as a function of simulation time. Here we only show $\lambda 8$ for the three independent replicates, additional $\lambda$ windows can be found in the SI. Sudden changes in $\mathrm{dH} / \mathrm{d} \lambda$ correlated with the entry/escape of water molecules into the space between protein and ligand. Water sampling was inadequate which shows that introducing the bubble-ligand only separates the water sampling from the decoupling of the ligand, but does not solve the problem. 
Table 7. Binding free energy $\Delta G^{\circ}$ for the HSP90 system using the bubble approach. We show the free energy difference of each individual leg of the thermodynamic cycle as well as the binding free energy calculated by summation along the cycle. Reported uncertainties are the standard deviation of three independent replicates. $\Delta G_{\text {restraints off }}$ was calculated analytically. ${ }^{52}$

\begin{tabular}{|l|l|l|}
\hline & EQ & NEQ \\
\hline \hline $\begin{array}{l}\Delta G_{\text {restraints on }} \\
{[\mathrm{kcal} / \mathrm{mol}]}\end{array}$ & \multicolumn{2}{|c|}{$1.26 \pm 0.04$} \\
\hline $\begin{array}{l}\Delta G_{\text {restrain loop }} \\
{[\mathrm{kcal} / \mathrm{mol}]}\end{array}$ & $1.8 \pm 0.2$ \\
\hline $\begin{array}{l}\Delta G_{\text {bubble on }} \\
{[\mathrm{kcal} / \mathrm{mol}]}\end{array}$ & $-6.979 \pm 0.006$ \\
\hline $\begin{array}{l}\Delta G_{\text {decouple ligand }} \\
{[\mathrm{kcal} / \mathrm{mol}]}\end{array}$ & $56.4 \pm 0.5$ & $56.2 \pm 0.3$ \\
\hline $\begin{array}{l}\Delta G_{\text {bubble off }} \\
{[\mathrm{kcal} / \mathrm{mol}]}\end{array}$ & $-10.6 \pm 0.2$ \\
\hline $\begin{array}{l}\Delta G_{\text {restraints loop off }} \\
{[\mathrm{kcal} / \mathrm{mol}]}\end{array}$ & $-3.1 \pm 0.5$ \\
\hline $\begin{array}{l}\Delta G_{\text {restraints off }} \\
{[\mathrm{kcal} / \mathrm{mol}]}\end{array}$ & -9.003 & \\
\hline $\begin{array}{l}\Delta G_{\text {solvate ligand }} \\
{[\mathrm{kcal} / \mathrm{mol}]}\end{array}$ & $-17.16 \pm 0.04$ & $-17.02 \pm 0.02$ \\
\hline$\Delta G^{0}[\mathrm{kcal} / \mathrm{mol}]$ & $-12.7 \pm 0.8$ & $-12.7 \pm 0.6$ \\
\hline
\end{tabular}

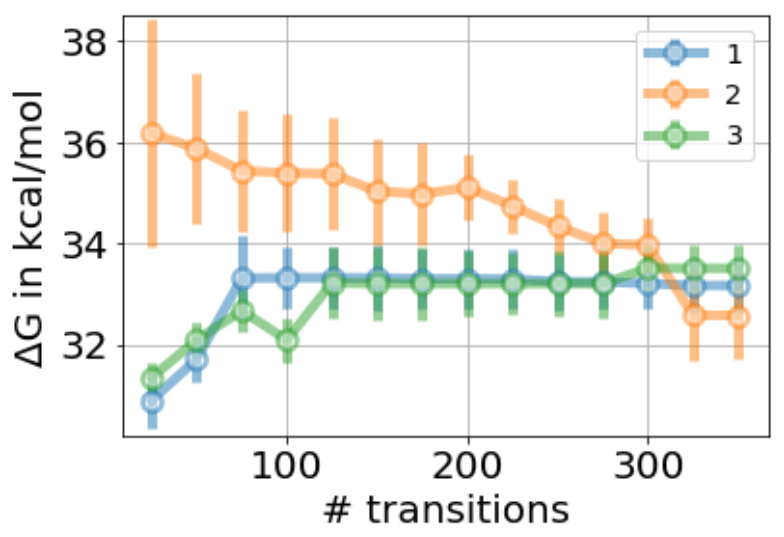

(a)

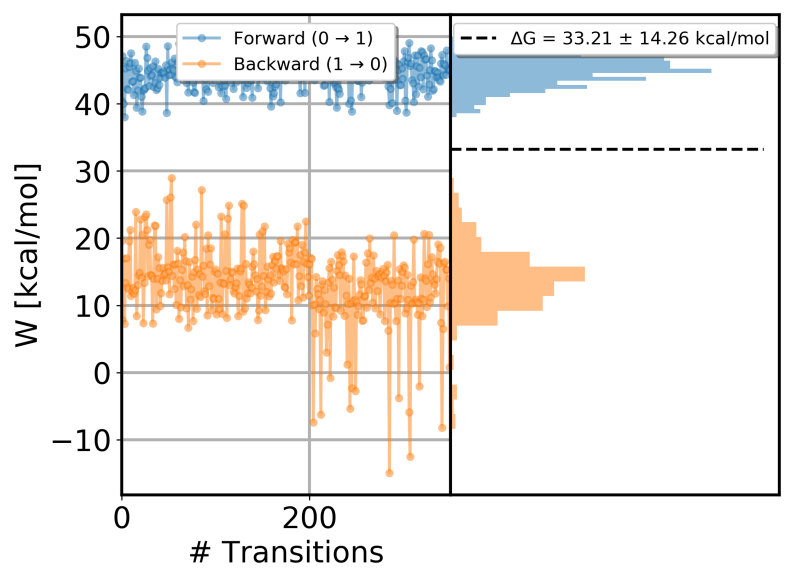

(b)

Figure S21. Weaker Boresch-style restraints $\left(5 \mathrm{kcal} /\left(\mathrm{mol} * \AA^{2}\right)\right.$ and $\left.5 \mathrm{kcal} /\left(\mathrm{mol} * \mathrm{rad}^{2}\right)\right)$. (a) Free energy difference for decoupling the HSP90 ligand in the binding site with the NEQ protocol using weaker Boresch-style restraints. The cumulative free energy difference is plotted against the number of transitions. Transitions were run for 1 ns. The standard deviation across three independent replicates was $0.4 \mathrm{kcal} / \mathrm{mol}$ which is higher than in the protocol with stronger force constant on the restraints $(0.2 \mathrm{kcal} / \mathrm{mol})$. (b) Work values for the ligand binding to HSP90. Shown are values for replicate 1 measured for each attempted transition, in forward (blue) and reverse (orange) direction, as well as the distribution of the work values. The reverse work distribution is similar to the one in the protocol using stronger restraints and the mean dissipation was similar in both protocols, in the reverse direction $18.7 \mathrm{kcal} / \mathrm{mol}$ for the protocol with a weaker force constant and $15.2 \mathrm{kcal} / \mathrm{mol}$ for the original protocol, and in the forward direction 11.5 vs. $13.9 \mathrm{kcal} / \mathrm{mol}$. 


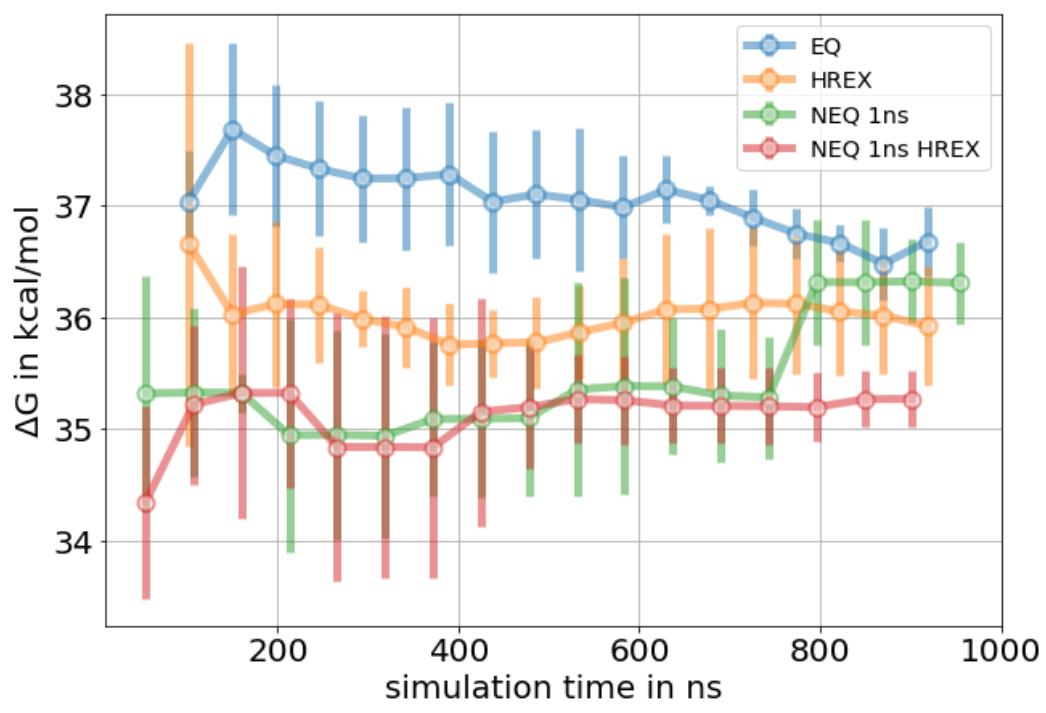

Figure S22. Free energy difference for decoupling the HSP90 ligand in the binding site. We show EQ and NEQ protocols with and without the use of HREX as an enhanced sampling technique. In the NEQ HREX approach, end state structures were used from the EQ HREX simulation, and no enhanced sampling was performed in the NEQ switches. For the EQ approach we show the mean free energy difference across three replicates while for the NEQ approach the free energy estimate was obtained by pooling work values from three replicates and estimating one free energy difference using BAR (Sec. 4.5). The uncertainty estimate in both approaches is the standard deviation across three replicates. Using structures from HREX simulations as input for the NEQ switches did not improve results in this case. On the contrary, additional sampling problems were introduced (see Figure S23).

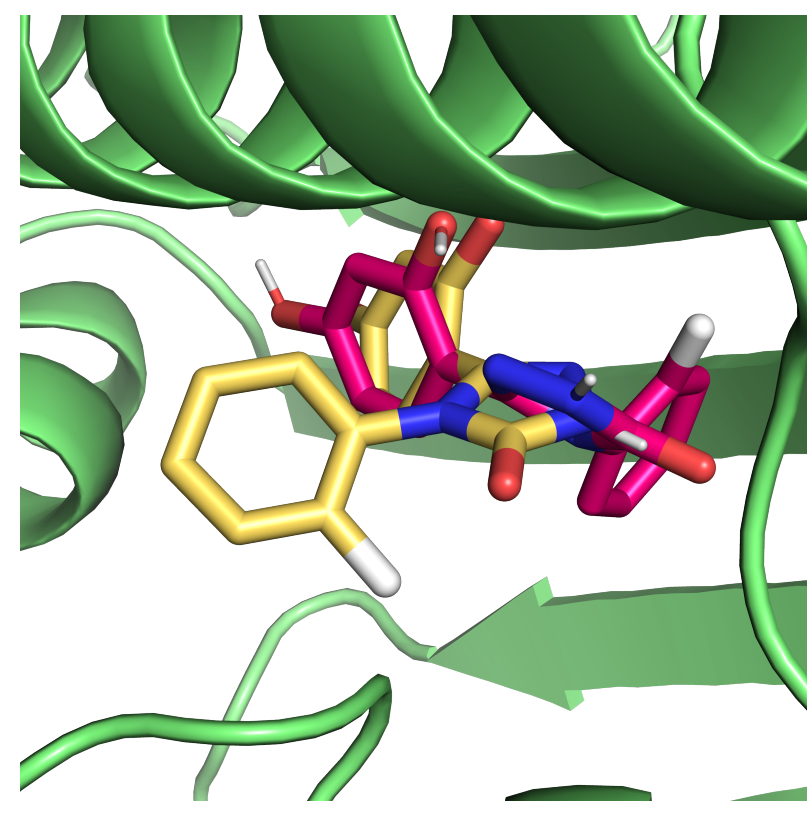

(a)

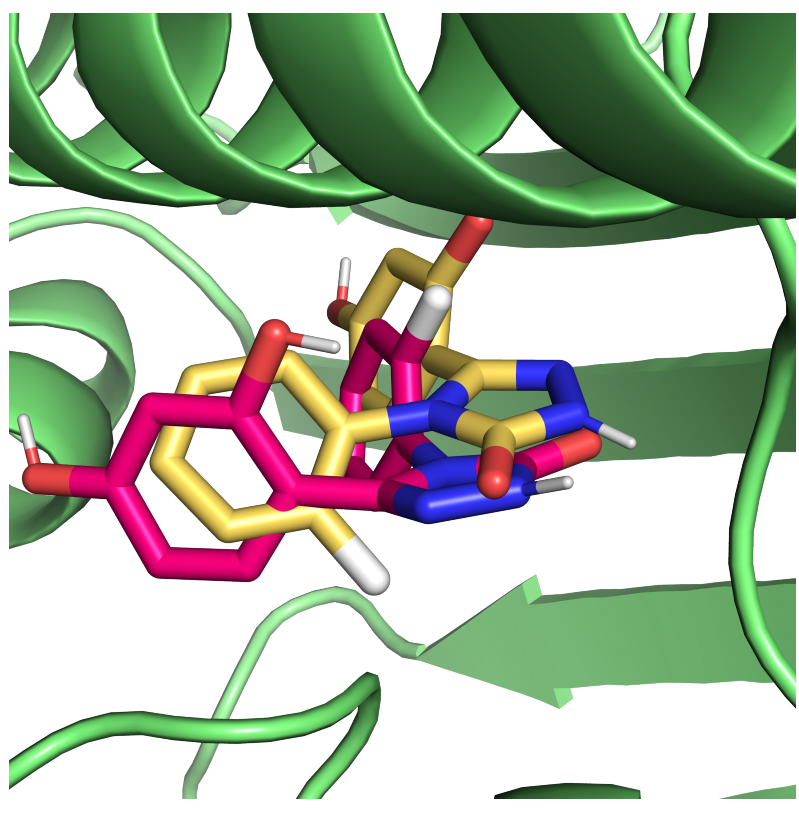

(b)

Figure S23. Flipped binding mode of the ligand binding to HSP90. The crystallographic binding pose is shown in yellow sticks, the flipped binding mode in magenta sticks. (a) In the non-interacting state the fluorophenyl ring was flipped in some frames. (b) Some NEQ switching transitions starting from the flipped binding mode in (a) crashed when the two phenyl rings flipped their position in the binding site. 
Graphical TOC Entry

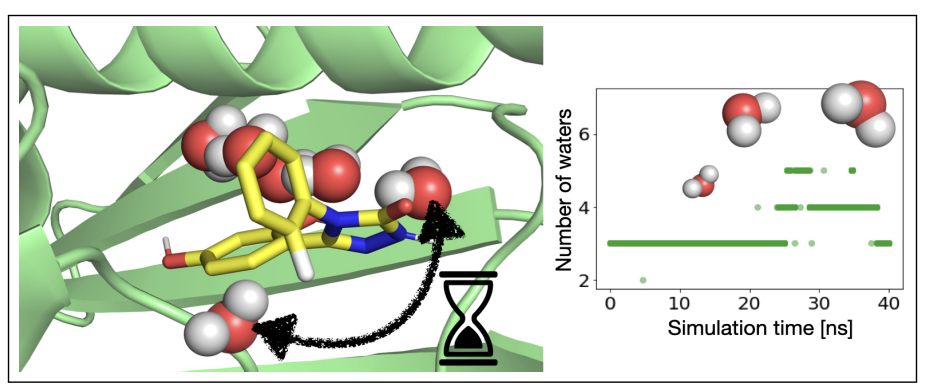

